\title{
Diving and Hyperbaric Medicine
}

The Journal of the South Pacific Underwater Medicine Society and the European Underwater and Baromedical Society

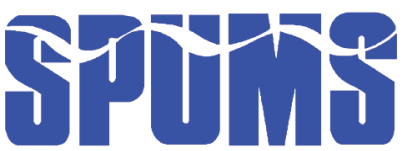

Volume 47 No. 3 September 2017
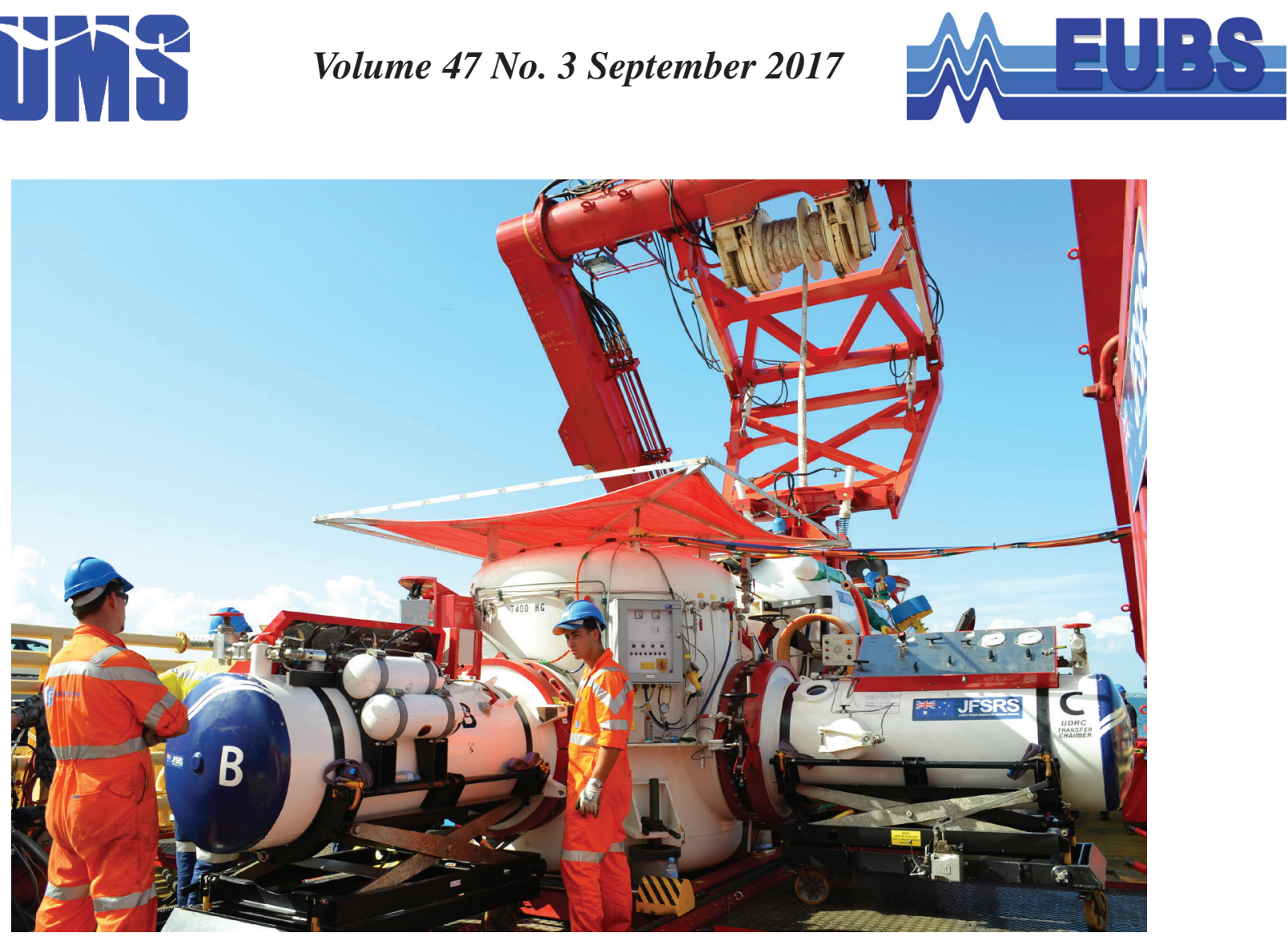

\section{Safely decompressing the rescuers}

What is the right heat for treating stonefish envenomation?

Understanding better the causes of diving fatalities

DAN members diving with medical conditions

More Turkish women are scuba diving

The dangerous marine environment includes salmon

A swollen face - don't dive with a cold!

Safety device for pleural drainage during hyperbaric treatment 


\title{
PURPOSES OF THE SOCIETIES
}

To promote and facilitate the study of all aspects of underwater and hyperbaric medicine

To provide information on underwater and hyperbaric medicine

To publish a journal and to convene members of each Society annually at a scientific conference

\section{SOUTH PACIFIC UNDERWATER MEDICINE SOCIETY}

\section{OFFICE HOLDERS}

President

David Smart

Past President Michael Bennett

Secretary

Douglas Falconer

Treasurer Sarah Lockley

Education Officer David Wilkinson

Chairman ANZHMG John Orton

Committee Members Jen Coleman Tamara Ford Ian Gawthrope Cathy Meehan Peter Smith

Webmaster Joel Hissink

Membership Steve Goble

\section{ADMINISTRATION}

<president@spums.org.au>

<pastpresident@spums.org.au>

$<$ secretary@spums.org.au>

$<$ treasurer@spums.org.au>

<education@spums.org.au>

<anzhmg@spums.org.au>

$<$ jh.coleman@me.com> $<$ drford.5frogs@gmail.com> <iangawthrope@yahoo.com> $<$ cmeehan@mcleodstmed.com.au> $<$ drpetersmith@bigpond.com>

<webmaster@spums.org.au>

\section{MEMBERSHIP}

For further information on SPUMS and to register to become a member, go to the Society's website: <www.spums.org.au>

The official address for SPUMS is:

c/o Australian and New Zealand College of Anaesthetists,

630 St Kilda Road, Melbourne, Victoria 3004, Australia

SPUMS is incorporated in Victoria A0020660B

\section{EUROPEAN UNDERWATER AND BAROMEDICAL SOCIETY}

\section{OFFICE HOLDERS}

President

Jacek Kot

Vice President

Ole Hyldegaard

Immediate Past President

Costantino Balestra

Past President

Peter Germonpré

Honorary Secretary Peter Germonpré

Member-at-Large 2016 Bengusu Oroglu

Member-at-Large 2015 Karin Hasmiller

Member-at-Large 2014 Robert van Hulst

Liaison Officer Phil Bryson

$$
\begin{aligned}
& \text { <jacek.kot@eubs.org> } \\
& \text { <ole.hyldegaard@eubs.org> } \\
& \text { <costantino.balestra@eubs.org> } \\
& \text { <peter.germonpre@eubs.org> } \\
& \text { <peter.germonpre@eubs.org> } \\
& \text { <bengusu.oroglu@eubs.org> } \\
& \text { <karin.hasmiller@eubs.org> } \\
& \text { <rob.van.hulst@eubs.org> } \\
& \text { <phil.bryson@eubs.org> }
\end{aligned}
$$

\section{ADMINISTRATION}

Honorary Treasurer and Membership Secretary Kathleen Pye

\section{MEMBERSHIP}

<secretary@eubs.org>

For further information on EUBS and to complete a membership application, go to the Society's website: <www.eubs.org> The official address for EUBS is:

Kathleen Pye

Chantrey, Hillside Road

Stromness, Orkney KW16 3HR, United Kingdom

EUBS is a UK Registered Charity No. 264970

\section{DIVING AND HYPERBARIC MEDICINE}

\author{
$<$ www.dhmjournal.com>
}

Editor:

Michael Davis <editor@dhmjournal.com>

PO Box 35

Tai Tapu 7645

New Zealand

Phone: +64-(0)3-329-6857

European (Deputy) Editor:

Lesley Blogg <euroeditor@dhmjournal.com>

Editorial Assistant:

Nicky Telles

Journal distribution:

Steve Goble

<editorialassist@dhmjournal.com>

<admin@spums.org.au>

Journal submissions:

Submissions should bemade at http://www.manuscriptmanager.com/dhm
Editorial Board:

Michael Bennett, Australia

David Doolette, USA

Christopher Edge, United Kingdom

Ingrid Eftedal, Norway

Peter Germonpré, Belgium

Jane Heyworth, Australia

Jacek Kot, Poland

Simon Mitchell, New Zealand

Claus-Martin Muth, Germany

Neal Pollock, Canada

Monica Rocco, Italy

Martin Sayer, United Kingdom

Erika Schagatay, Sweden

David Smart, Australia

Robert van Hulst, The Netherlands 


\section{The Editor's offering}

\section{Diving fatalities}

The anecdotal reporting of diving fatalities of yesteryear has moved to a steadily more structured approach by investigating authorities such as the police, pathologists and conroners. In New Zealand (NZ), for instance, the police use a standardised, detailed report document in all cases. For many years, forensic pathologists in Australia and NZ have been encouraged to use the protocol promulagated by the Royal College of Pathologists of Australia for the conduct of diving fatality autopsies. ${ }^{1}$ Modern imaging for the detection of gases in tissues has improved, novel gas sampling techniques are starting to be used, ${ }^{2}$ and we have a better understanding of the mechanisms of gas formation within the diver cadaver. ${ }^{3}$ Christopher Lawrence, a forensic pathologist in Hobart, Tasmania, gave several interesting talks on these issues at the SPUMS ASM in 2016, Perhaps it is time to update the autopsy protocol?

Accurate reporting is the first step in understanding the epidemiology of diving fatalities. The second step is to bring the various components together in structured reports to better understand the causes of diving fatalities and what steps might be taken to reduce their numbers. ${ }^{4,5}$ This presents considerable challenges, particularly since incident reports are often patchy and confused or, in the case of unwitnessed solo dives, largely absent. In this issue, Lippmann et al. report their efforts "to further develop and better define a process for performing a CEA [chain of events analysis] to reduce potential subjectivity and increase consistency between analysts". ${ }^{6}$ The complexity of these exercises is well illustrated in their paper. Nevertheless, this approach does offer the possibility of a better insight into the evolution of a fatal diving incident.

The third step would be instituting changes in diving practices to reduce morbidity and mortality in recreational diving. This is even more difficult to study. Take, for example, the fact that the majority of dead scuba divers are found with their weights system still in place. Weights ditching procedures are taught to all trainee divers, yet in an emergency, this is rarely performed even by divers who reach the surface conscious. Is this because it is taught badly, not practiced enough, reluctance by the diver, a decision that is overwhelmed by panic or excessive task loading or simply that by the time it is needed the diver is too incapacitated? I am not aware of a serious analysis of this sad statistic and how it might be reversed.

Nevertheless, changes in dive training procedures have improved safety in the past. When the writer learnt to dive with the British Sub-Aqua Club in the early 1960s, a bouyant ascent with continuous exhalation, after ditching one's weights, was taught and performed in open water. There were plenty of anecdotal reports of air embolism and some deaths with this technique, but no useful incidence rate data. Thirty years later at the time of a SPUMS Workshop on emergency ascent training, the Professional Association of Diving Insrtuctors (PADI) reported only two fatalities during training in nearly four million controlled swimming ascents, with the regulator in the mouth. ${ }^{7}$

One does need to keep all this in perspective - recreational scuba diving is a safe sport, but when things go wrong they go wrong seriously, hence the focus on fatalities.

\section{Tricon2018}

Next year our two societies combine again with the South African Underwater and Hyperbaric Medicine Association for a conference in Durban, South Africa (see page 205). Tricon2013 in Reunion was a great success scientifically and socially, providing a great opportunity for colleagues from all over the world to meet. The 2018 meeting will be no less successful. Tricon2013 was not particularly well attended by SPUMS members and I encourage as many of you as possible to attend this time around.

\section{References}

1 Lawrence C, Cooke C. Autopsy and the investigation of scuba diving fatalities. Guidelines from the Royal College of Pathologists of Australia; 2003. SPUMS Journal. 2006;36:2-8. [cited 2017 August 12]. Available from: http://archive.rubicon foundation. org/xmlui/bitstream/ handle/123456789/10163/DHM_V36N1_2.pdf?sequence=1.

2 Varlet V, Dominguez A, Augsburger M, Lossois M, Egger C, Palmiere C, Vilarino R, Grabherr S. Understanding scuba diving fatalities: carbon dioxide concentrations in intra-cardiac gas. Diving Hyperb Med. 2017;47:75-81.

3 Edmonds C, Caruso J. Diving fatality investigations: recent changes. Diving Hyperb Med. 2014;44:91.6.

4 Lippmann J, Lawrence CL, Fock A, Jamieson S, Harris R. Provisional report on diving-related fatalities in Australian waters in 2011. Diving Hyperb Med. 2016;46:207-40.

5 Lippmann J, Baddeley A, Vann R, Walker D. An analysis of the causes of compressed gas diving fatalities in Australia from 1972-2005. Undersea Hyperb Med. 2013;40:49-61.

6 Lippmann J, Stevenson C, Taylor D McD, Williams J, Mohebbi M. Chain of events analysis for a scuba diving fatality. Diving Hyperb Med. 2017;47:144-54.

7 Richardson D, Cummins T. A training agency perspective of emergency ascent training. SPUMS Journal. 1993;23:225-30.

Key words

Editorials; Diving deaths; Investigations; Emergency ascent

Michael Davis

Front-page photo, taken on MV Seahorse Horizon, is of James Fisher personnel preparing to mobilise a one-man recompression chamber from the transfer-under-pressure unit (TUP) of the Australian Navy's DISSUB system. The TUP is interlocked with the LR5 Submersible Rescue Vehicle (photo courtesy of Mark Carey, GM JFD Australia). 


\section{The Presidents' pages}

\section{Jacek Kot, President EUBS}

You are receiving this copy of the Journal just before or immediately after our EUBS Annual Scientific Meeting. Therefore, I cannot use this letter to send you any messages for the conference, nor am I able to summarize the meeting. What I can say for sure is that it will, as always, be interesting. All 42 previous EUBS conferences were interesting, so why should this one be different? Especially as it is to be held in Ravenna, Italy! Instead, I decided to share with you two cautionary tales which I found in recent publications concerning deaths of divers inside hyperbaric chambers.

One very recent death, in late June of this year, was that of a 61-year-old coral diver in a portable hyperbaric chamber on board the diving vessel, close to Girona in Spain. ${ }^{1}$ The chamber exploded, also injuring two others on board, one seriously. As usual at the beginning of any investigation, information is scarce and not explanatory. We hope soon to have access to more information or at least to have the opportunity to hear from experts involved in the official investigation. This is especially important since similar cases have occurred worldwide in the past. It is essential that we continue our mission of supporting any local authorities in understanding that hyperbaric chambers per se can be lethal devices, not only for the people inside, but also for those in the vicinity, especially when operated by lay users outside of a hospital setting. In Europe according to the directive for medical devices, hyperbaric chambers are classified as socalled Class IIB devices which means that they are "intended to administer medicines by means of a delivery system (...) in a manner that is potentially hazardous taking account of the mode of application". ${ }^{2}$ This directive concerns medical installations, but pressure is the same, oxygen is the same and most hazards are the same whatever the setting.

The second case, this time from outside Europe, concerns the death of a diver referred to a hyperbaric facility with a clinical diagnosis of "DCS Type 1 and CO poisoning". ${ }^{3}$ Description of the case clearly indicates that these diagnoses were incorrect, as the diver presented with cardiopulmonary and other symptoms of severe decompression illness from the very beginning. The most striking information is that he was placed in a multiplace chamber as the sole occupant without an inside-chamber attendant and underwent a US Navy Treatment Table 5. The authors describe that "when the chamber was opened 150 minutes after the start of recompression therapy, the patient was found without vital signs". The paper presents discussion about the diagnosis and possible scenarios and everyone interested in diving and hyperbaric medicine should read it. I do not claim that, if there had been an inside attendant, the diver would have survived; there is insufficient information within this paper to draw such a conclusion and the severe symptoms with which the diver presented indicated a life-threating disorder.

What I wish to emphasize is that such cases remind us why we strongly support the concept of having multiplace chambers with trained medical or nursing personnel inside the chamber. This debate is nothing new, especially in selected, elective patients, but this is a timely reminder to all my hyperbaric medical colleagues that hyperbaric chambers are Class IIB medical devices (see above). We should all know the risks, list the hazards and propose mitigating measures to reduce those risks for patients, medical personnel and by-standers. Nevertheless, fatal accidents still occur in hyperbaric chambers (in recent times in Indonesia, Italy, Mexico, South Africa, Spain and the USA), so everyone must remain alert!

\section{References}

1 Murcia today. Exploding hyperbaric chamber kills Girona coral fisherman. [cited 2017 July 30]. Available at: https:// murciatoday.com/exploding-hyperbaric-chamber-kills-gironacoral-fisherman_122788-a.html.

2 Council Directive 93/42/EEC of 14 June 1993 concerning medical devices. Official Journal of the European Communities no L 169/1 - 169/43 - 14 June 1993.

3 Mendez N, Huchim-Lara O, Rivera-Canul N, Chin W, Tec J, Cordero-Romero S. Fatal cardiopulmonary decompression sickness in an untrained fisherman diver in Yucatan, Mexico: a clinical case report. Undersea Hyperb Med, 2017;44: 279-81.

Key words

Medical society, General interest, Hyperbaric facilities; Fire or explosion; Case reports

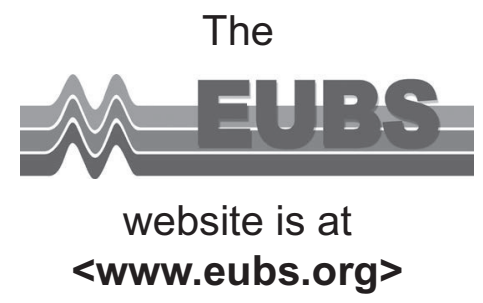

Members are encouraged to log in and keep their personal details up to date. 


\section{David Smart, President SPUMS}

The 46th SPUMS Annual Scientific Meeting (ASM) held in Bali this year was a great success and a credit to conveners Katie Commons and Clinton Gibbs and scientific convener Denise Blake. Our international speakers Debbie Pestell, Jürg Wendling and Neal Pollock and Ultrasound Workshop supervisor Ian Gawthrope provided an excellent foundation for many other speakers on the conference theme of occupational diving. The venue at Rama Candidasa was ideal for our conference requirements with terrific food, a high standard of amenities and outstanding service from staff. In addition, travel linkages were smooth, assisted by Diversion Dive travel, and the practical diving workshops of a high standard from Tauch Terminal and Bali Diving Academy. We were grateful for support from the Office of Naval Research, USA, towards speaker costs. Our meeting was linked to the Asian Hyperbaric and Diving Medicine Association, which offered good networking opportunities with our Asian Colleagues. Numbers at this year's conference were close to 150 , a great result, and our largest attendance in recent years. Thanks to all concerned.

\section{SPUMS ASMs - volunteers to convene future ASMs}

A key part of SPUMS' mission and aims is to convene members of the Association annually at a scientific conference and to develop fellowship and friendship amongst its members. This year's meeting achieved that aim with conspicuous success. It is important that we maintain this momentum. Cathy Meehan is chair of the SPUMS "Future ASM Committee". This committee generates ideas for future ASM venues, undertakes research regarding their viability, then presents the options for the SPUMS Executive Committee (ExCom) to choose the best option. The Future ASM committee is open for any SPUMS member to join - we particularly need volunteers as conveners for future ASMs, commencing 2019. Conveners' costs are covered if they volunteer to run an ASM.

SPUMS has specific criteria for the ASM and has welldeveloped guidelines to follow. Future ASMs need venues that can accommodate up to 150 delegates, which are located a single sector from major Australian (and New Zealand) cities, can cater for 100 divers, have adequate conference facilities and offer value for money. Once every 4-5 years, a more exotic destination, with more complex travel is accessed (e.g., Palau). Once the criteria are worked through, the number of venues that can accommodate our unique style of ASM is quite limited. On that basis, it is likely that we will return to some venues regularly, because they tick the right boxes.

In addition, once every 4-5 years, SPUMS will join our European and South African colleagues for the Tricontinental conference. The last one held on Reunion Island in 2013 was very successful; however, SPUMS member attendance was low (by SPUMS ASM standards), at around 40 delegates. In 2018, the second Tri-continental meeting will be held in Durban, South Africa. I have very recently signed a Memorandum of Understanding on behalf of SPUMS to organise and participate in the meeting to be held 23-28 September, 2018. This meeting will be in lieu of the SPUMS ASM next year, and presents a great opportunity to mix with our colleagues from around the world, be exposed to cutting edge diving and hyperbaric medical science and to explore another part of the world. I encourage all SPUMS members to make the extra effort to attend.

\section{Election of Society Officers}

The Annual General Meeting 2017 was the first SPUMS general election since we adopted our new Purposes and Rules. Our 2017-2020 committee was elected as follows:

\author{
President - David Smart \\ Immediate Past President - Mike Bennett \\ Secretary - Douglas Falconer \\ Treasurer - Sarah Lockley \\ General Member - Cathy Meehan \\ General Member - Jenny Coleman \\ General Member - Peter Smith \\ General Member - Tamara Ford \\ General Member - Ian Gawthrope
}

The following ex officio positions are due for expressions of interest and new appointments (current appointees shown)
ANZHMG Chair - John Orton (retired) (elected by the ANZHMG)
Webmaster - Joel Hissink
Education Officer - David Wilkinson
Journal Editor - Mike Davis (retiring 2018)

The new ANZHMG Chairperson will be elected at the ANZHMG AGM in Adelaide on 17 August. A call for expressions of interest for Webmaster and Education Officer has been circulated to all members and successful candidates will be appointed once the EOIs are reviewed. I offer my sincere thanks to the previous committee, and outgoing members Simon Mitchell, John Orton, Denise Blake and Joel Hissink. An appointee to succeed Mike Davis as Editor of Diving and Hyperbaric Medicine is currently under negotiation by SPUMS and EUBS.

Finally - SPUMS NEEDS MORE MEMBERS, AND MEMBER SUPPORT WITH RECRUITMENT! Membership numbers are currently around 450. We have targeted 500 as a goal by the end of the year. Please encourage doctors whom you know, who are interested in diving medicine, to join SPUMS.

\section{Key words}

Medical society; General interest; Meetings 


\title{
Original articles Chain of events analysis for a scuba diving fatality
}

\author{
John Lippmann ${ }^{1,2}$, Christopher Stevenson², David McD Taylor ${ }^{3,4}$, Jo Williams², \\ Mohammadreza Mohebbi ${ }^{5}$
}

\author{
${ }^{1}$ Divers Alert Network (DAN) Asia-Pacific, Ashburton, Victoria, Australia \\ ${ }^{2}$ School of Health and Social Development, Deakin University, Geelong, Victoria, Australia \\ ${ }^{3}$ Emergency Department, Austin Hospital, Victoria, Australia \\ ${ }^{4}$ Department of Medicine, University of Melbourne, Australia \\ ${ }^{5}$ Biostatistics Unit, Deakin University, Geelong, Victoria, Australia
}

Corresponding author: John Lippmann, Divers Alert Network (DAN) Asia-Pacific, PO Box 384, Ashburton, Victoria 3147 , Australia

johnl@danap.org

Key words

Scuba diving; Incidents; Deaths; Investigations; Analysis; Epidemiology

\begin{abstract}
(Lippmann J, Stevenson C, Taylor D McD, Williams J, Mohebbi M. Chain of events analysis for a scuba diving fatality. Diving and Hyperbaric Medicine. 2017 September;47(3):144-154.)

Introduction: A scuba diving fatality usually involves a series of related events culminating in death. Several studies have utilised a chain of events-type analysis (CEA) to isolate and better understand the accident sequence in order to facilitate the creation of relevant countermeasures. The aim of this research was to further develop and better define a process for performing a CEA to reduce potential subjectivity and increase consistency between analysts.

Methodology: To develop more comprehensive and better-defined criteria, existing criteria were modified and a template was created and tested using a CEA. Modifications comprised addition of a category for pre-disposing factors, expansion of criteria for the triggers and disabling agents present during the incident, and more specific inclusion criteria to better encompass a dataset of 56 fatalities. Four investigators (raters) used both the previous criteria and this template, in randomlyassigned order, to examine a sample of 13 scuba diver deaths. Individual results were scored against the group consensus for the CEA. Raters' agreement consistency was compared using the Index of Concordance and intra-class correlation coefficients (ICC).

Results: The template is presented. The index of concordance between the raters increased from 62\% (194/312) using the previous criteria to $82 \%(257 / 312)$ with use of this template indicating a substantially higher inter-rater agreement when allocating criteria. The agreement in scoring with and without template use was also quantified by ICC which were generally graded as low, illustrating a substantial change in consistency of scoring before and after template use.

Conclusion: The template for a CEA for a scuba diving fatality improves consistency of interpretation between users and may improve comparability of diving fatality reports.
\end{abstract}

\section{Introduction}

Some accident investigations utilise a root cause analysis (RCA) technique. This follows the 'domino mechanism'1 whereby an initial causal factor leads to other factors ending in injury or death. However, it can be difficult to determine a single causal factor as the more thoroughly an incident is investigated, the more potential causal factors appear. ${ }^{2}$

A scuba diving fatality usually involves a series of related events culminating in death. Identification of various contributory factors is hampered by the reality that most diving incident reports are relatively sparse on detail. Given the difficulty in tracking a root cause, such an analysis is more appropriately described and conducted as a "chain of events' or 'sequential analysis' where there may be multiple possibilities at some of the stages.

The process of chain of events analysis (CEA) was first applied to diving incidents in the examination of 947 fatalities in the USA. ${ }^{3}$ The incident sequence was divided into four components: (1) the trigger; (2) the disabling agent; (3) the disabling injury and (4) the cause of death. Later, CEA was applied to a series of 351 Australian compressedgas diving fatalities. ${ }^{4}$ Modifications included the re-defining of several subcategories within the four major categories of the CEA to better reflect the available data. Subsequently, others have used similar categories for their analyses. ${ }^{5}$ However, in the absence of definitive criteria, there is a potential for subjectivity in the categorisation of events 
Table 1

Predisposing factors

Definition: A predisposing factor (as used here) is a relevant factor that was present prior to the dive, and/or prior to the trigger occurring, and which is believed to have predisposed to the incident and/or to key components in the accident chain (e.g., the trigger or disabling agent).

Health-related: May include factors such as a history of cardiovascular disease, epilepsy, diabetes as well as factors such as mental or physical fatigue.

Organisational/training/experience/skills-related: Includes factors that may have impacted a diver's practical readiness to dive. Factors such as the suitability of training course design and conduct and the overall dive organisation by a dive operator are included as well as the level of skill and/or experience of the diver relevant to the circumstances.

Planning-related: Applies to obviously poor pre-dive planning decisions, whether made well before the dive or immediately prior. This includes factors such as a decision to dive in conditions that were obviously unsuitable, or should have reasonably have been assessed as unsuitable or a decision to dive alone, among others.

Poor communication or coordination: If communication between buddies and/or the dive supervisor (prior to or during the dive) is poor, the opportunity of misunderstandings and unexpected and inappropriate actions is increased.

Absence of appropriate equipment; using obviously faulty equipment: Although this is somewhat planning-related, this particular category is equipment-specific.

Activity-related: Some activities (e.g., spearfishing/seafood collecting, penetration diving) are associated with particular inherent risks. For example, spearfishing is well known to attract sharks and can predispose to an accident triggered by the arrival of an aggressive shark. Penetration diving can predispose to an incident triggered by entrapment.

Unsafe supervision: This can apply to supervision by the divemaster overseeing a dive, an instructor supervising students or to a diver's interactions with his buddy.

Other: Includes anything that cannot appropriately be allocated to one of the defined categories. This category should be used sparingly and only after a serious attempt to utilise an existing category.

Unknown/none: There is insufficient information on which to make a reasonable suggestion of a possible predisposing factor.

and classifications can vary substantively from case to case and study to study. A detailed breakdown of predisposing factors, triggers, disabling agents and disabling injuries are presented in Tables $1-4$.

To minimise subjectivity and increase consistency between investigators, it is important to carefully define the CEA categories. The aim of this study was to design a template to assist researchers to more objectively categorise the components of a CEA and so facilitate greater compatibility within and between diving incident studies. Where sufficient information is available, the template also provides the opportunity to identify and include some of the human factors which may have influenced events prior to the dive and during some segments of the chain of events. More consistent reporting will help to better inform the diving industry of various contributors to diving injuries and so enable the identification of appropriate countermeasures to help mitigate future deaths.

\section{Methods}

Access to data for this study was approved by the Justice Human Research Ethics Committee, Department of Justice, Victoria, Australia (No. CF/06/31). The program of research was also approved by the Human Research Ethics Committee at Deakin University, Victoria, Australia (No. 2013-210).

\section{TEMPLATE CREATION}

Each of the 54 Australian scuba diving fatalities from 2004 to 2010 was re-examined and a CEA applied, using the previous criteria., ${ }^{4,6-12}$ A sequence category of 'pre-disposing factors' was added, the criteria for the other sequential event categories were expanded, and more specific inclusion criteria were created to better encompass the dataset. As a result, a broader yet better-defined template for the CEA was constructed. 


\section{TEMPLATE ASSESSMENT AND ADJUSTMENT}

The resulting template was subsequently utilised by four investigators experienced in analysing diving deaths in the examination of 13 of the 14 scuba diving fatalities that occurred in Australia in 2011, for which the usual coronial reports were available at the time. ${ }^{13}$ The process involved the following steps:

- Careful scrutiny of the available data on the relevant medical and diving history of the victim and circumstances of each incident;

- consideration of the possible causative factors and chain of events;

- comparison with the categories and subcategories of the available template;

- $\quad$ selection and recording of the preferred categories and subcategories.

For example, in deciding whether there were any potential predisposing factors, the investigator considered the victim's health, training, experience and skills as well as the planning and supervision of the dive, the equipment used and the nature of the activity. If there was no obvious predisposing factor, it was marked as 'unknown/none'. If a possible factor was identified which did not align with a defined category, it was allocated to 'Other' and noted for further consideration of template modification.

Two investigators were randomly assigned to perform an initial analysis of each incident without using the template (but using the previous criteria). The other two used the template. Subsequently, the roles were reversed and those who initially used the template were asked to conduct a second analysis using the original criteria, while the others used the template. Analyses were conducted two to four weeks apart. All results were collated, discussed and a consensus was reached for the final CEA to be used with this fatality series. The template was further adjusted to reflect feedback received and the final template is presented under results.

The Index of Concordance (IC) ${ }^{14}$ between investigators was calculated with and without template use. The IC is defined as the number of inter-rater agreements divided by the number of coding attempts and multiplied by 100 to yield a percentage. ${ }^{14}$ The IC between investigators when using the template was calculated and an IC of greater than $70 \%$ was taken as indicating agreement. ${ }^{15}$ In addition, each rater's selection was compared to the consensus selections and graded either ' 1 ' if in agreement or ' 0 ' if not. In this manner, a score of $0-4(0=$ no agreement; $4=$ all raters matched consensus) was given for each of the categories for each incident. These scores were summed to create a total incident score.

The Intraclass Correlation Coefficient (ICC(2)) was calculated for each category of the CEA and for the total incident score, comparing these scores with and without use of the template. In a repeated measures ANOVA involving four raters and 13 subjects, ICC(2) assumes both raters and subjects are random effects. Cut-off values of $<0.7,0.7-0.9$ and $>0.9$ have been considered as poor, acceptable and good agreement. ${ }^{16}$

\section{Results}

\section{TEMPLATE DESCRIPTION}

The major sequence categories and their definitions in this CEA were as follows:

- Predisposing factor: A relevant factor(s) that was present prior to the dive, and/or prior to the trigger occurring, and which was believed to have predisposed to the incident and/or to key components in the accident chain (e.g., the trigger or disabling agent);

- Trigger: The earliest identifiable event that appeared to transform an unremarkable dive into an emergency;

- Disabling agent: An action or circumstance (associated with the trigger) that caused injury or illness, e.g., an action of the diver or other persons, function of the equipment, effect of a medical condition or a force of nature;

- Disabling injury: Injury or condition directly responsible for death or incapacitation followed by death from drowning;

- Cause of death: As specified by the medical examiner, which could be the same as the disabling injury or could be drowning secondary to injury.

Note that, although the disabling injury is often more informative in determining why the diver became incapacitated, the actual cause of death can sometimes provide important information for preventative and emergency management strategies.

Suggested sub-categories for each of the categories are shown in Tables 1-4, whilst Table 5 provides illustrative examples of how these taxonomies may be used. Figure 1 provides a summarised flowchart for a CEA.

\section{TEMPLATE ASSESSMENT}

The index of concordance between the raters when using the template was $82 \%$ (257/312) compared with $62 \%$ $(194 / 312)$ when not using it. This absolute IC difference of $20 \%$ is substantial and clinically significant. Table 6 shows the comparison of the raters' agreement with and without template use for each scuba-related category and total score.

Table 7 shows the mean and standard deviation (SD) scores with each category of the CEA both with (T) and without (NT) template use, as well as the ICC(2) for each category and for the total scores. As it is illustrated, all post-template mean scores are higher with smaller SDs, indicating a higher agreement and less between-raters heterogeneity. Three out of four categories had poor agreement consistency, with an 
Table 2

Triggers

Definition: A trigger (as used here) is the earliest identifiable event that appeared to transform an unremarkable dive into an emergency.

Environment-related: Relates to the diving environment and may arise from the topography or conditions of the dive site itself or as a result of contact with other users or inhabitants.

- Conditions

Water - e.g., difficulty in dealing with current, swell, surge, chop, water outflow/inlet; visibility, temperature;

Weather - e.g., problem arising from rain, wind, lightning;

- Marine animal contact - e.g., aggression from shark;

- Watercraft-related - e.g., boat impact, propeller injury;

- Entrapment - e.g., tangled in line or net, disoriented in cave or wreck;

- Events triggered from the direct effects of immersion, submersion and/or sensory deprivation, e.g., cardiac arrhythmia.

Equipment-related: Includes a problem with any item of the diver's equipment that precipitates an accident. The list below is not exhaustive and other equipment may trigger an accident and so can be included.

- Regulator, surface-supplied breathing apparatus or rebreather functional problem leading to gas supply reduction or interruption;

- Buoyancy compensator device (BCD) failure - e.g., sticky inflator, dump valve failure but not including its misuse;

- Weights-related - e.g., unintentional release, unable to be released but not including over- or under-weighting from the outset of the dive;

- Mask - e.g., leak, broken strap, broken/displaced lens;

- $\quad$ Fins - e.g., poor fit, broken strap, loss of fin;

- $\quad$ Exposure suit - e.g., tight wetsuit, flooded drysuit but not including drysuit 'blow-up';

- Cylinder - e.g., valve-failure, slippage but not including problems from gas content or valve insufficiently opened;

- Faulty depth or contents gauge;

- Hose failure;

- Breathing hose entanglement.

Gas supply-related: Includes any problem relating to the on-going supply, purity and suitability of the breathing gas but not loss of gas supply due to equipment failure.

- Inappropriate breathing gas mixture;

- Contamination;

- Exhaustion of breathing gas supply.

Buoyancy-related: Includes buoyancy problems generally related to poor knowledge or skills but not problems arising from equipment failure. It includes factors such as:

- Overweighted or underweighted;

- Poor skills;

- Drysuit 'blow-up';

- Loss of buoyancy control arising from deployment of surface marker buoy.

Exertion-related: Includes problems arising from situations such as carrying equipment, exiting the water post-dive, dragging a heavy object underwater, etc. but not exertion as a result of sea conditions.

Anxiety/stress-related: Anxiety/stress can often be associated with diving, especially in the relatively inexperienced, and can be a trigger for an accident. However, in order for this to be listed as a trigger, it cannot be assumed but must have been observed and reported by a witness.

Primary diver error: Diver error, which is an inherent part of many diving accidents, can be a precursor to or the actual trigger in a sequence. It can be closely associated with another trigger(s) and, in such cases, can be reported in combination.

Other: Includes anything that cannot appropriately be allocated to one of the defined categories. This category should be used sparingly and only after a serious attempt to utilise an existing category.

Unknown: There is insufficient information on which to make a reasonable suggestion of a possible trigger(s). 
Table 3

Disabling agents

Disabling agents in a scuba diving fatality

Definition: A disabling agent (as used here) is an action or circumstance (associated with the trigger) that caused injury or illness. It may be an action of the diver or other persons, reaction of the equipment, effect of a medical condition or a force of nature.

Gas supply-related: Includes any problem relating to the on-going supply, purity and suitability of the breathing gas but not loss of gas supply due to equipment failure.

- Inappropriate breathing gas mixture;

- Contamination;

- Exhaustion of breathing gas supply.

Ascent-related: Includes any problem that was likely precipitated by or associated with the ascent from a dive. This may include but is not restricted to:

- Breath holding during ascent;

- Gas sharing during ascent;

- Rapid ascent.

Medical-related: Includes any problem that was likely precipitated by or associated with a pre-existing or imminent medical condition (which may or may not be the same as the disabling injury). It may include but is not restricted to:

- Cardiovascular disease;

- Other medical condition.

Buoyancy-related: Includes buoyancy problems related to poor knowledge or skills and secondary to some trigger. It can also include loss of buoyancy control subsequent to equipment failure (e.g., loss of buoyancy subsequent to a faulty BCD).

- Inadequate buoyancy control underwater;

- Lack/loss of buoyancy on surface;

- Drysuit 'blow-up' subsequent to equipment failure.

Environment-related: Includes problems where a diver is disabled as a result of environmental circumstances such as:

- Adverse sea conditions;

- Entrapment - this entrapment is secondary to an initial trigger such as narcosis, silting, poor buoyancy or surge. It often leads to exhaustion of the breathing gas (in which case, the disabling agent is recorded as environmental - entrapment, then out of breathing gas.

- Impact with watercraft, rocks, reef;

- Dangerous marine animal contact (e.g., shark attack).

Equipment-related: Includes a consequence of a problem associated with an item of the diver's equipment, but secondary to some accident trigger.

Other: Includes anything that cannot appropriately be allocated to one of the defined categories. This category should be used sparingly and only after a serious attempt to utilise an existing category.

Unknown: There is insufficient information on which to make a reasonable suggestion of a possible disabling agent.

ICC(2) less than 0.70 illustrating poor agreement between before and after template use. This illustrates a systematic improvement in between-rater scores after template implementation.

\section{Discussion}

In this study, the use of the template improved consensus in the evaluation of the diving fatalities as indicated by the large increase in IC when using the template. This was supported by the relatively low ICC(2) ratings which indicate a marked difference in scoring (consistency of agreement) with and without template use. An ICC of 0.70-0.90 may be acceptable for use in research purposes but not for policymaking. ${ }^{17}$

An advantage of using ICC(2) to compare measurement methods is that it can be used when the measurements are given on different scales or metrics, as the $\operatorname{ICC}(2)$ is a dimensionless ratio. Because reliability depends on the heterogeneity of the true error-free values in the sampled population, it is essential that reliability 


\section{Table 4}

Disabling injuries

Disabling injuries and causes of death

Definition: A disabling injury (as used here) is directly responsible for death or incapacitation followed by death from drowning.

Asphyxia: Asphyxia with or without aspiration of water and with no indication of a prior disabling injury

Cerebral arterial gas embolism: Gas in the cerebral arteries with or without evidence of lung rupture

Cardiac: Acute chest discomfort indicated by the diver, history of cardiac disease, or autopsy findings

Trauma: Witnessed trauma, traumatic findings at autopsy

Other medical: Stroke, gastrointestinal haemorrhage, carbon monoxide toxicity, etc

Other: This includes anything that cannot appropriately be allocated to one of the defined categories. This category should be used sparingly and only after a serious attempt to utilise an existing category.

Unknown: Body not recovered; no autopsy available; no indications of disabling injury at autopsy.

\section{Cause of death}

Definition: The cause of death is that specified by the medical examiner, which could be the same as the disabling injury or could be drowning secondary to injury.

Although the disabling injury is often more informative in determining why the diver became incapacitated, the actual cause of death can sometimes provide important information for preventative and emergency management strategies.

ICC(2)s are compared only if they have been estimated from the same population. ${ }^{18}$ Usually, assessments made by two different raters are less similar than are two assessments made by the same rater. Assessments from two raters may differ systematically due to bias between the raters (a rater 'effect'), and their measurement errors may also have different SDs. For example, assessments from a rater who can make more precise assessments will have a smaller SD than those made by a less precise rater. Since assessments in the future are to be made by different raters, we need to describe and quantify the differences between such assessments in order to judge whether differences are genuine or may be due to measurement error. As such, the ideal way to study this was for each rater to make at least two assessments of a sample of subjects. The design of such a study and the type of appropriate statistical analyses was guided by the fact that our interest lay in drawing inferences about a wider population of potential raters, not only the particular set of raters. Due to the fact that the raters in the study are considered a random sample from the population of potential raters, we analysed the study using a model that treats the rater 'effect' as a random effect. Thus, we used a two-way random-effects model, with random subject effects and random observer effects.

Although factors such as experience and medical history, where known, are included, the traditional sequential analysis for diving-related injury largely failed to address other human factors often associated with such incidents.
In a study of 1,000 (mainly non-fatal) diving incidents, The Diver Incident Monitoring Survey (DIMS) reported that $87 \%$ of the incidents were associated with diver error. ${ }^{19}$ Given the inhospitable environment in which diving takes place, in addition to adequate health and fitness, the foundation for safe diving includes 'human factors' such as adequate knowledge and skill acquisition and retention and clear, often rapid decision-making. A CEA may provide a useful tool to identify 'how' an incident occurred, i.e., the sequence of events in the dive incident itself. However, preceding and/ or underlying many of the components in such an analysis is the potential for diver error or inappropriate behaviour from human factors.

Using techniques from research into aviation accidents, a Human Factors Analysis and Classification System (HFACS) was applied to recreational scuba diving incidents in an attempt to determine 'why' they occurred..$^{20,21}$ The HFACS comprised two major divisions: 'active failures' (unsafe acts) which involved "diver error", and 'latent failures', which included factors that occurred prior to the incident and which influenced the active failure. A "Swiss cheese model" 22 was used to argue that a dive incident does not simply result from diver error alone but is ultimately the result of the alignment of several 'holes' in each of the layers of latent and active failures.

This protocol is more readily applicable to non-fatal incidents where greater detail is often available and where the diver 
Table 5

\section{PREDISPOSING FACTORS}

Examples of the use of the CEA template - predisposing factor, triggers and disabling agents

\section{Health-related:}

- A diver with significant cardiovascular disease may be predisposed to a cardiac event with immersion and/or exertion.

- A diver who is intoxicated is at an increased risk of making poor decisions.

Organisational/training/experience/skills-related:

- A dive organisation offers services to non-English-speaking (NES) clientele. A NES group booked for a dive and no arrangements were made to translate the dive briefing into the clients' language. As a result, several divers act contrary to the brief and one subsequently dies.

- A person untrained or poorly trained in the use of a buoyancy compensator device may be more likely to have a buoyancy-related problem, so triggering an accident.

Planning-related:

- A diver went diving alone and without a lookout to retrieve a craypot. He became entangled in a line and was unable to free himself.

- When two divers arrived at their planned dive site, although there were large waves constantly breaking over the entry and exit points from the rocks, they decided to dive there anyway. When trying to enter the water, one diver was swept off the ledge, struck his head on rocks and drowned.

Poor communication or co-ordination:

- One of a buddy pair was aware of a strong current on the other side of a 'swim-through', but failed to communicate this to the other, who subsequently swam into the current and was swept away.

Absence of appropriate equipment or using obviously faulty equipment:

- A diver who dives without a BCD may be predisposed to a buoyancy-related problem.

- A diver who dives with air from a faulty or poorly maintained compressor may be predisposed to contaminated air.

Unsafe supervision:

- Poor supervision by the divemaster and/or buddy may result in an inexperienced diver entering the water without all equipment in place and functional and so predispose to an accident.

\section{TRIGGERS}

Environment-related:

- A diver got into difficulties while struggling to make headway against a strong current.

Trigger $=$ Environment-related (current)

- A spearfisherman was confronted by an aggressive shark.

Predisposing: Activity-related (collecting seafood).

Trigger $=$ Environment-related $($ shark $)$

- A diver breathing air at a depth of 55 metres' sea water was suffering severe narcosis, misread his contents gauge and ran out of air.

Predisposing: Activity-related (deep air diving)

Trigger $=$ Environment-related (narcosis at depth)

Equipment-related:

- A diver's BCD inflator became stuck open resulting in a buoyant ascent.

Trigger $=$ Equipment-related (sticky BCD inflator)

- A diver's regulator failed, causing a loss of air supply.

Trigger $=$ Equipment-related (regulator failure)

\section{Gas supply-related:}

- A technical diver using a rebreather became unconscious at depth due to hypercapnia resulting from overloading of the $\mathrm{CO}_{2}$ scrubber.

Predisposing $=$ Faulty equipment $\left(\mathrm{CO}_{2}\right.$ scrubber inadequate $)$

Trigger $=$ Gas supply-related $\left(\mathrm{CO}_{2}\right.$ scrubber exhausted $)$

- A diver ran out of breathing gas while trying to complete required decompression and was forced to make a rapid ascent.

Predisposing $=$ Poor planning (unless something unpredictable occurred)

Trigger $=$ Gas supply-related (out of gas)

Buoyancy-related:

- After deploying his surface marker buoy (SMB), a diver became entangled in its line and was dragged to the surface.

Trigger $=$ Buoyancy-related (SMB entanglement)

- An inexperienced drysuit user became inverted, was unable to dump air from the suit and had an uncontrolled ascent.

Predisposing = Experience-related (inexperienced)

Trigger $=$ Buoyancy-related (drysuit blow-up)

Exertion-related:

- A (healthy) diver collecting abalone became exhausted and distressed while dragging his heavy catch bag and then 
struggled to stay afloat on reaching the surface.

Trigger = Exertion-related (heavy catch bag)

\section{Anxiety/stress-related:}

- A student on an introductory dive was seen to panic and rush to the surface after encountering a large stingray.

Predisposing $=$ Experience-related (inexperience)

Trigger $=$ Anxiety/stress-related (panic from stingray encounter)

\section{Primary diver error:}

- A rebreather diver on the surface forgot to turn off her bailout valve before removing her mouthpiece. Water entered the scrubber and when she replaced the mouthpiece and breathed from the unit she suffered a 'caustic cocktail'.

Trigger $=$ Diver error (equipment-related)

- A diver forgot to open his tank valve before jumping in. Being negatively buoyant, he sank without an available air supply.

Trigger $=$ Diver error (gas supply-related)

\section{DISABLING AGENTS}

Gas supply-related:

- A diver became entangled in a line inside a wreck, was unable to free herself and ran out of air.

Predisposing = Activity-related (wreck penetration)

Trigger $=$ Environmental (entanglement)

Disabling agent: Gas supply-related (out of gas)

\section{Ascent-related:}

- A preoccupied underwater photographer failed to check his air, ran out of air, held his breath during ascent and suffered a pulmonary barotrauma.

Trigger $=$ Gas-supply-related (out of air)

Disabling agent: Ascent-related (breath-holding)

\section{Medical-related:}

- Faulty oxygen sensors in a rebreather enabled the $\mathrm{PO}_{2}$ to rise sufficiently to cause a hyperoxic convulsion in the diver.

Predisposing = Equipment fault (old/poorly-calibrated oxygen sensors)

Trigger $=$ Gas supply-related (incorrect breathing gas mix from sensor failure)

Disabling agent: Medical-related (hyperoxic convulsion)

- A diver with a history of epilepsy is seen to become unconscious and have a seizure during a shallow air dive.

Predisposing $=$ Health-related (epilepsy)

Trigger $=$ Environmental (sensory effects)

Disabling agent: Medically-related (seizure)

\section{Buoyancy-related:}

- A diver surfaced in rough conditions after losing a fin due to a broken fin strap. He was negatively buoyant, failed to replace his regulator, inflate his BCD or ditch weights and was swamped by a wave and sank.

Trigger = Equipment-related (torn fin strap)

Disabling agent: Buoyancy-related (lack of buoyancy on surface)

- A drysuit inflator stuck open causing over-inflation and inversion in the water. During the process, the diver aspirated some water and became unconscious.

Trigger $=$ Equipment-related (drysuit inflator malfunction)

Disabling agent: Buoyancy-related (inversion underwater)

\section{Environment-related:}

- A diver lost control of her buoyancy, ascended into and became entangled in the shotline and subsequently ran out of air.

Trigger $=$ Buoyancy-related (poor buoyancy control)

Disabling agent: Environment - entrapment (out of air)

- A diver in rough water was thrown against rocks by a large wave, hit his head and became unconscious.

Trigger $=$ Environmental (rough conditions)

Disabling agent: Environmental (head impact with rocks)

- An abalone diver was approached and subsequently attacked by a shark.

Predisposing $=$ Activity-related (collecting seafood)

Trigger $=$ Environmental (aggressive shark)

Disabling agent: Environmental - shark attack

Equipment-related:

- A rebreather diver on the surface momentarily forgot to turn off her bailout valve before removing her mouthpiece, enabling water ingress. The water entered the scrubber and when she replaced the mouthpiece and breathed from the unit she suffered a 'caustic cocktail'.

Trigger $=$ Diver error (equipment-related).

Disabling agent: Equipment-related ('caustic cocktail'). 
Table 6

Comparison of investigator scores of scuba deaths with (T) and without template (NT) use; note that the score is not intended to be used when investigating a diving accident; it was just a simple method to try to quantify any gross difference in score allocation by the raters with and without template use; it does not represent how the data is used

\begin{tabular}{|c|c|c|c|c|c|c|c|c|c|c|}
\hline \multirow[t]{2}{*}{ Case } & \multicolumn{2}{|c|}{ Predisposing factor } & \multicolumn{2}{|c|}{ Trigger } & \multicolumn{2}{|c|}{ Disabling agent } & \multicolumn{2}{|c|}{ Disabling injury } & \multicolumn{2}{|c|}{ Total $(* / 16)$} \\
\hline & NT & $\mathrm{T}$ & NT & $\mathrm{T}$ & NT & $\mathrm{T}$ & NT & $\mathrm{T}$ & NT & $\mathrm{T}$ \\
\hline 1 & 4 & 4 & 3 & 4 & 4 & 4 & 3 & 4 & 14 & 16 \\
\hline 2 & 3 & 3 & 1 & 3 & 4 & 4 & 4 & 3 & 12 & 13 \\
\hline 3 & 3 & 4 & 3 & 3 & 3 & 4 & 4 & 4 & 13 & 15 \\
\hline 4 & 3 & 4 & 3 & 4 & 1 & 1 & 3 & 4 & 10 & 13 \\
\hline 5 & 4 & 4 & 2 & 4 & 3 & 4 & 3 & 4 & 12 & 16 \\
\hline 6 & 2 & 4 & 2 & 3 & 2 & 3 & 3 & 4 & 9 & 14 \\
\hline 7 & 3 & 4 & 2 & 4 & 2 & 3 & 2 & 3 & 9 & 14 \\
\hline 8 & 3 & 4 & 3 & 4 & 4 & 4 & 4 & 4 & 14 & 16 \\
\hline 9 & 3 & 4 & 2 & 3 & 3 & 4 & 4 & 4 & 12 & 15 \\
\hline 10 & 4 & 4 & 3 & 4 & 4 & 4 & 4 & 4 & 15 & 16 \\
\hline 11 & 1 & 3 & 2 & 2 & 2 & 4 & 4 & 4 & 9 & 13 \\
\hline 12 & 3 & 3 & 3 & 3 & 3 & 3 & 4 & 4 & 13 & 13 \\
\hline 13 & 2 & 2 & 2 & 4 & 3 & 4 & 3 & 4 & 10 & 14 \\
\hline
\end{tabular}

Table 7

Mean (SD) scores for category and total with (T) and without (NT) template use; ICC(2) - intraclass correlation coefficient; $\mathrm{CI}$ - confidence intervals

\begin{tabular}{lclllcl} 
Category & \multicolumn{2}{c}{ NT } & \multicolumn{2}{c}{ T } & ICC (2) & $(95 \%$ CI) \\
Predisposing factor & 2.9 & $(0.9)$ & 4.0 & $(1.0)$ & 0.68 & $(0-0.90)$ \\
Trigger & 2.4 & $(0.7)$ & 3.54 & $(0.66)$ & 0.49 & $(0-0.85)$ \\
Disabling agent & 2.9 & $(1)$ & 3.54 & $(0.88)$ & 0.86 & $(0.53-0.96)$ \\
Disabling injury & 3.46 & $(0.66)$ & 3.85 & $(0.38)$ & 0.42 & $(0-0.82)$ \\
Total (out of 16) & 11.7 & $(2.1)$ & 14.5 & $(1.27)$ & 0.73 & $(0.13-0.92)$
\end{tabular}

can provide feedback and personal insight into the various human factors involved. However, sometimes witness reports and other background information can indicate where, and which human factors contributed to the cascade of events leading to the death of a diver. So the addition of known or reasonably suspected human factor considerations to a CEA of diving fatalities is valuable in order to obtain a fuller picture of the entire scenario. Such human factors include pre-existing health conditions, inadequate training and skills, inappropriate/poorly-functional equipment; or more deeply-rooted organisational problems that may underpin some of these. An example of an underlying organisational problem is an inadequate training focus and practice in weight-ditching. Given the large number of diving fatality victims whose bodies are found with their weights still in situ, ${ }^{5,23}$ this is an important area to address from training agency level down.

Another important organisational-level consideration is improved education about how certain co-existing conditions can impact on diving safety. Pre-certification diving medical examinations are now uncommon in many countries, with an increasing reliance on a diver to honestly and accurately answer a self-reporting medical questionnaire. This places a greater onus on dive professionals and existing or intending divers to understand the questions and the associated implications, and to take these seriously and act accordingly. This has become increasingly evident with the increasing representation of divers with pre-existing medical conditions (especially cardiac-related) and older divers in dive fatality reports. ${ }^{4,13,24}$

In reality, multiple factors, discrete or linked, may simultaneously influence an action or circumstance within an incident. It is important for the investigator to logically and systematically consider all possibilities and influences when trying to determine a possible sequence of events. This can be a daunting task, prone to substantial subjectivity and variation between investigators.

When developing this template, one of the main aims was to reduce the variability in categorisation in existing protocols in order to make determinations more uniform between assessors and so increase comparability both within and between studies. This was done by increasing specificity within the categories. In addition, where possible, the categories were designed to be mutually exclusive and as encompassing as practicable to minimise the need to record a component as 'other' and so improve the consistency. ${ }^{25}$

A certain degree of subjectivity is inescapable given the frequent gaps in the information available to diving 
Figure 1

Flowchart of chain of events analysis of a scuba diving accident

\begin{tabular}{|ll|}
\hline & Predisposing factors \\
- & Health \\
& Organisational/training/ \\
- & Planning \\
- & Poor communication or \\
& co-ordination \\
- & Equipment \\
- & Activity \\
- & Unsafe supervision \\
- & Other \\
\hline
\end{tabular}

\begin{tabular}{|ll|}
\hline & \multicolumn{1}{c}{ Triggers } \\
- & Environmental \\
$\bullet$ & Equipment \\
$\bullet$ & Gas supply \\
- & Buoyancy \\
- & Exertion \\
- & Anxiety/stress \\
- & Primary diver error \\
- & Other \\
\hline & Unknown \\
\hline
\end{tabular}

\begin{tabular}{|ll|}
\hline & Disabling agents \\
$\bullet$ & Gas supply \\
$\bullet$ & Ascent \\
- & Medical \\
- & Buoyancy \\
- & Environment \\
- & Other \\
- & Unknown \\
\hline
\end{tabular}

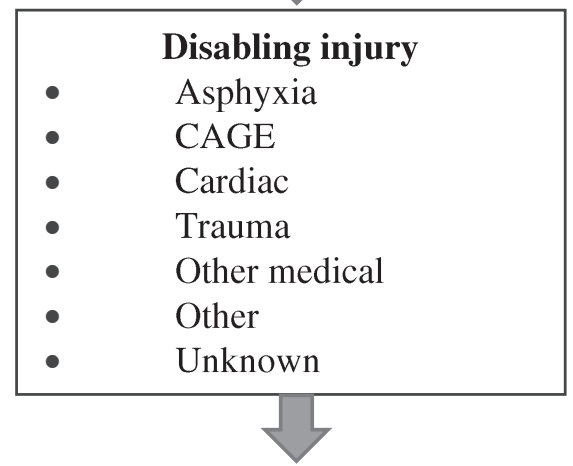

Cause of death

As determined by medical examiner incident investigators. However, by creating taxonomies that achieve reasonable consensus between users, subjectivity is reduced. To this end, it remains important to test the consistency between users when allocating events using such taxonomies. There are a variety of suggested methods for determining this but the IC is reportedly the industry standard for use with safety data. ${ }^{26}$

The study did highlight several minor categorisation problems, which were adjusted in the template presented here. Undoubtedly, more improvements will be identified with further use and the template will need to be modified accordingly. Users are encouraged to send feedback to the corresponding author.

\section{LIMITATIONS}

There was likely a net learning effect to both the raters who used the template first and those who did not. Despite the delay between allocations, those who did not use it initially may have been more familiar with the case by the time they re-examined it. Similarly, those who used it first may have recollected some of the categorisation within the template. The template allocations were mixed in an attempt to balance these issues and likely served to narrow the difference between allocations with and without the template. Given the limited information that is often available, and the continued challenges associated with the subjectivity and categorisation of aspects of diving deaths, there will inevitably be variation between reporters.

\section{Conclusions}

A CEA can be useful in diving fatality investigations to identify the likely sequences of events that lead to the divers' demise. However, there is often limited information on which to base such an analysis and this can lead to substantial variation in the interpretation of events by different investigators. An effective template for allocating the components of a chain of events may be useful in reducing the variability between investigators within a study and increase the comparability of different diving fatality studies. Although still imperfect and likely to be modified with future use, this CEA template has been shown to improve consistency of interpretation between users.

\section{References}

1 Heinrich HW. Industrial Accident Prevention. New York: McGraw Hill; 1936. p. 37.

2 Wallace B, Ross A. Beyond human error - taxonomies and safety science. Boca Raton, FL: Taylor and Francis; 2006. p. 25.

3 Denoble PJ, Caruso JL, de L Dear G, Vann RD. Common causes of open-circuit recreational diving fatalities. Undersea Hyperb Med. 2008;35:393-406.

4 Lippmann J, Baddeley A, Vann R, Walker D. An analysis of the causes of compressed gas diving fatalities in Australia from 
1972-2005. Undersea Hyperb Med. 2013;40:49-61.

5 Vinkel J, Bak P, Hyldegaard O. Danish diving fatalities 1999-2012. Diving Hyperb Med. 2016;46:142-9.

6 Lippmann J, Lawrence C, Fock A, Jamieson S. Provisional report on diving-related fatalities in Australian waters 2010. Diving Hyperb Med. 2015;44:154-75.

7 Lippmann J, Lawrence C, Wodak T, Fock A, Jamieson S. Provisional report on diving-related fatalities in Australian waters 2009. Diving Hyperb Med. 2013;43:194-217.

8 Lippmann J, Lawrence C, Wodak T, Fock A, Jamieson S, Walker D, Harris R. Provisional report on diving-related fatalities in Australian waters 2008. Diving Hyperb Med. 2013;43:16-34.

9 Lippmann J, Walker D, Lawrence C, Fock A, Wodak T, Jamieson S. Provisional report on diving-related fatalities in Australian waters 2007. Diving Hyperb Med. 2012;42:151-70.

10 Lippmann J, Walker D, Lawrence C, Fock A, Wodak T, Jamieson S. Provisional report on diving-related fatalities in Australian waters 2006. Diving Hyperb Med. 2011;41:70-84.

11 Walker D, Lippmann J, Lawrence C, Fock A, Wodak T, Jamieson S. Provisional report on diving-related fatalities in Australian waters 2005. Diving Hyperb Med. 2010;40:131-49.

12 Walker D, Lippmann J, Lawrence C, Houston J, Fock A. Provisional report on diving-related fatalities in Australian waters 2004. Diving Hyperb Med. 2009;39:138-61.

13 Lippmann J, Lawrence C, Fock A, Jamieson S. Provisional report on diving-related fatalities in Australian waters 2011. Diving Hyperb Med. 2016;46:207-40.

14 Ross AJ, Wallace B, Davies JB. Technical note: measurement issues in taxonomic reliability. Saf Sci. 2004;42:771-8.

15 Wallace B, Ross A. Beyond human error - taxonomies and safety science. Boca Raton, FL: Taylor and Francis; 2006. p. 86.

16 Shrout PE, Fleiss JL. Intraclass correlations: uses in assessing rater reliability. Psychological Bulletin. 1979;86:420-8.

17 Martins WP, Nastri CO. Interpreting reproducibility results for ultrasound measurements. Ultrasound Obstet Gynecol. 2014;43:479-80.

18 Bland JM, Altman DG. Statistical methods for assessing agreement between two methods of clinical measurement. Lancet. 1986;1:307-10.

19 Acott CJ. Human error and violations in 1,000 diving incidents: a review of data from the Diving Incident Monitoring study (DIMS). SPUMS Journal. 2005;35:11-7.

20 Lock G. Human factors within recreational scuba diving - an application of the human factors analysis and classification system (HFACS). 21 March 2011. [cited 2016 August 10]. Available from: https://cognitasresearch.files.wordpress. com/2012/08/human-factors-in-sport-diving-incidents.pdf

21 Lock G. 2014 DISMS Annual Report. Cognitas Incident and Management Ltd. 01 Feb 2015. [cited 2016 Sept 27]. Available from: https://cognitasresearch.files.wordpress.com/2015/02/2 014dismsannualreport_final.pdf.

22 Reason J. Human error: models and management. BMJ (Clinical Research Ed). 2000;320:768-70.

23 Lippmann J. Diving deaths downunder. In: Vann RD, Lang MA, editors. Recreational diving fatalities. Proceedings of the Divers Alert Network 2010 April Workshop. Durham, NC: Divers Alert Network; 2011; p. 86-97.

24 Denoble PJ, Pollock NW, Vaithiyanathan P, Caruso JL, Dovenbarger JA, Vann RD. Scuba injury death rate among insured DAN members. Diving Hyperb Med. 2008;38:182-8.

25 Wallace B, Ross A. Beyond human error - taxonomies and safety science. Boca Raton, FL: Taylor and Francis; 2006. p. 48.

26 Wallace B, Ross A. Beyond human error - taxonomies and safety science. Boca Raton, FL: Taylor and Francis; 2006. p. 93.

\section{Acknowledgements}

The authors are grateful to Chris Lawrence, Andrew Fock and Scott Jamieson for their help in the scoring exercises, and to Petar Denoble for his comments on the draft.

Submitted: 02 December 2016; revised 12 April 2017 Accepted: 23 June 2017 


\title{
Heat deactivation of the stonefish Synanceia horrida venom - implications for first-aid management
}

\author{
Samantha Barnett, Silvia Saggiomo, Michael Smout, Jamie Seymour \\ Australian Institute of Tropical Health and Medicine, James Cook University, Cairns, Queensland, Australia \\ Corresponding author: Silvia Saggiomo, Australian Institute of Tropical Health and Medicine, James Cook University, \\ 14-88 McGregor Road, Smithfield, Cairns, Queensland 4878, Australia \\ silvia.saggiomo@my.jcu.edu.au
}

Key words

Marine animals; Toxins; Envenomation; First aid; Water; Temperature; Myocytes, cardiac

\begin{abstract}
(Barnett S, Saggiomo S, Smout M, Seymour J. Heat deactivation of the stonefish Synanceia horrida venom - implications for first-aid management. Diving and Hyperbaric Medicine. 2017 September;47(3):155-158.)

Objectives: To investigate the effects of temperature and hot water immersion time on neutralising venom lethality of the Australian estuarine stonefish (Synanceia horrida).

Design: Depths of the spines were measured while venom was extracted from $S$. horrida individuals. The venom was then exposed to temperatures of $4^{\circ} \mathrm{C}, 37.0^{\circ} \mathrm{C}, 40.1^{\circ} \mathrm{C}, 42.3^{\circ} \mathrm{C}, 45.0^{\circ} \mathrm{C}, 47.7^{\circ} \mathrm{C}, 55.2^{\circ} \mathrm{C}$, and $60.0^{\circ} \mathrm{C}$ for either five or 20 minutes incubation periods. Venom samples were added to cultured human cardiomyocytes and cell viability curves were produced using the ACEA's xCELLigence real-time cell monitoring system.

Main outcome measures: Determination of venom lethality on cardiomyocytes at a range of temperatures.

Results: The average depth of the spine required to go into a victims' flesh before the venom gland compressed and expelled venom was $18 \mathrm{~mm}$. Cardiomyocytes exposed to heat-treated venom for five minutes required higher temperatures to neutralise $99 \%$ of the venom, namely $44.6^{\circ} \mathrm{C}$ in comparison to $42.1^{\circ} \mathrm{C}$ with an incubation time of 20 minutes.

Conclusion: This study supports the use of hot water immersion therapy in the treatment of S. horrida stings. It is suggested that due to the depth of the puncture wound longer incubation times should be sought to allow heat to penetrate the deeper portions of the dermis and effectively begin venom deactivation.
\end{abstract}

\section{Introduction}

Hot water immersion (HWI) therapy has been the principal first-aid treatment employed for the alleviation of pain in fish envenomation injuries throughout the past two centuries. ${ }^{1}$ HWI treatment has shown to be effective across a large suite of piscine families, perhaps the most notable being the highly venomous stonefishes (Synanceiidae family). ${ }^{2-6}$ Despite the duration of time HWI therapy has been practiced and the fact that it appears to be effective, no detailed studies have been performed to analyze how temperature and exposure time influence the lethal behaviour of fish venom.

Envenomation from stonefishes occurs when force is applied to the integument sheath that encases the dorsal spine of the animal, along with the compression of the dual venom sacs on either side of the spine. The venom sacs can be a third to a half of the length of the spine and subsequently excrete venom along a venom duct in the spine and into the contacting body. ${ }^{7,8}$ Despite injuries being minor for the most part, severe scenarios have been documented. The symptoms of envenomation encompass immediate and radiating pain, appreciable local morbidity and paralysis, gross oedema, headache and, in severe cases, hypotension, bradycardia, arrhythmia, heart failure and death. ${ }^{9-12}$
Studies suggest that owing to the proteinaceous nature of the venom, ${ }^{13-20}$ heating the solution will cause deactivation of the protein components and thus reduce the venom activity. It has been observed at temperatures of $50^{\circ} \mathrm{C}$ that venom toxicity is neutralized; ${ }^{20}$ however, treating patients with water at this temperature could result in skin burns and tissue necrosis. ${ }^{21}$ Current treatment protocols suggest that the victim be treated with HWI around $42-45^{\circ} \mathrm{C}$ for 30 to 90 minutes (min). ${ }^{22,23}$ This is a generalised procedure done for stings or stabs produced by stingrays, starfish, sea urchins, weeverfish, scorpionfish and stonefish. ${ }^{11}$ To date, no activity range for stonefish venom and its relationship with heat have been produced, thus no protocols specifically exist for stonefish envenomations.

Consequently, this study aims to investigate the thermo-labile behaviour of Synanceia horrida venom. More specifically, we explored temperatures that provide therapeutic benefit for treating stonefish envenomations achieved by examining the effect of venom toxicity with varying temperatures and heat exposure times.

\section{Methods}

Venom was collected from mature $S$. horrida housed at the James Cook University Cairns Campus research aquarium 
Figure 1

The Australian estuarine stonefish (Synanceia horrida)

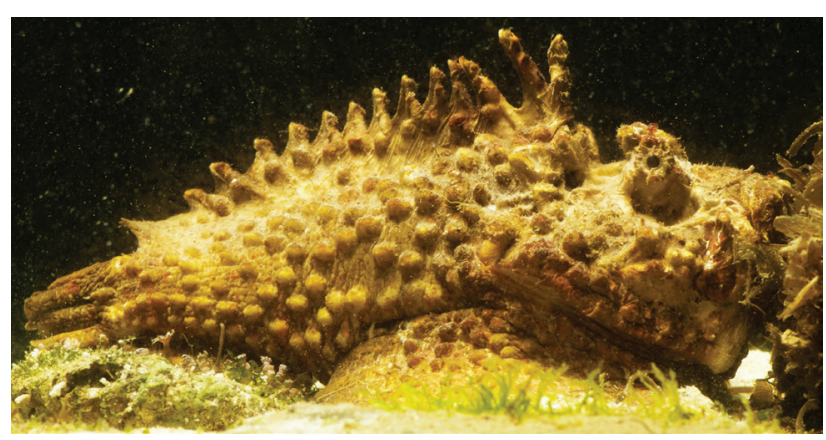

Figure 3

High resolution photographs of the dorsal spine of the Australian estuarine stonefish (Synanceia horrida) being compressed with a rubber strip: A - downwards pressure first being applied to spine; $\mathrm{B}$ - integument sheath surrounding the venom gland has been compressed and venom is being released from the hollow duct through the spine; $\mathrm{C}$ - venom gland is compressed and venom is still being released under pressure; D - venom gland fully compressed and has been emptied of its volume

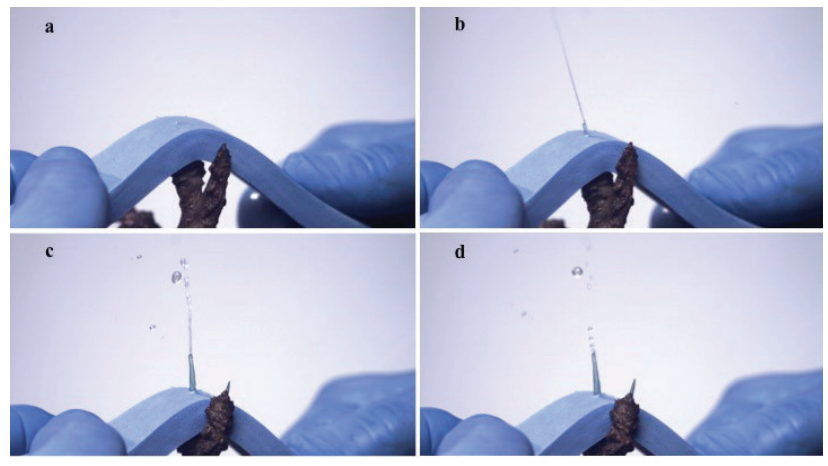

Figure 2

The intact exposed venom apparatus (dorsal spine and paired venom glands) of the Australian estuarine stonefish (Synanceia horrida)

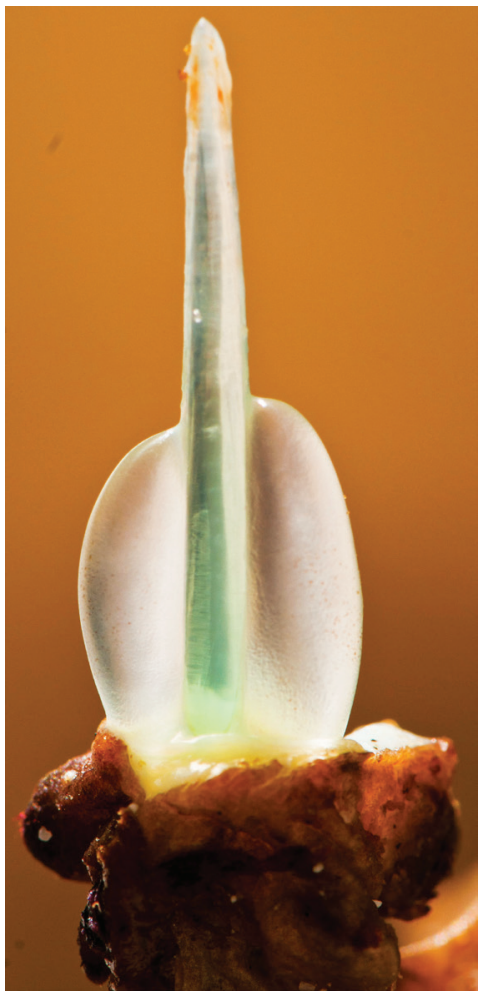

\section{Figure 4}

Cell survival of human cardiomyocytes when exposed to Synanceia horrida venom at different temperatures for two different incubation times (5 and 20 minutes); error bars represent standard deviation in replicates

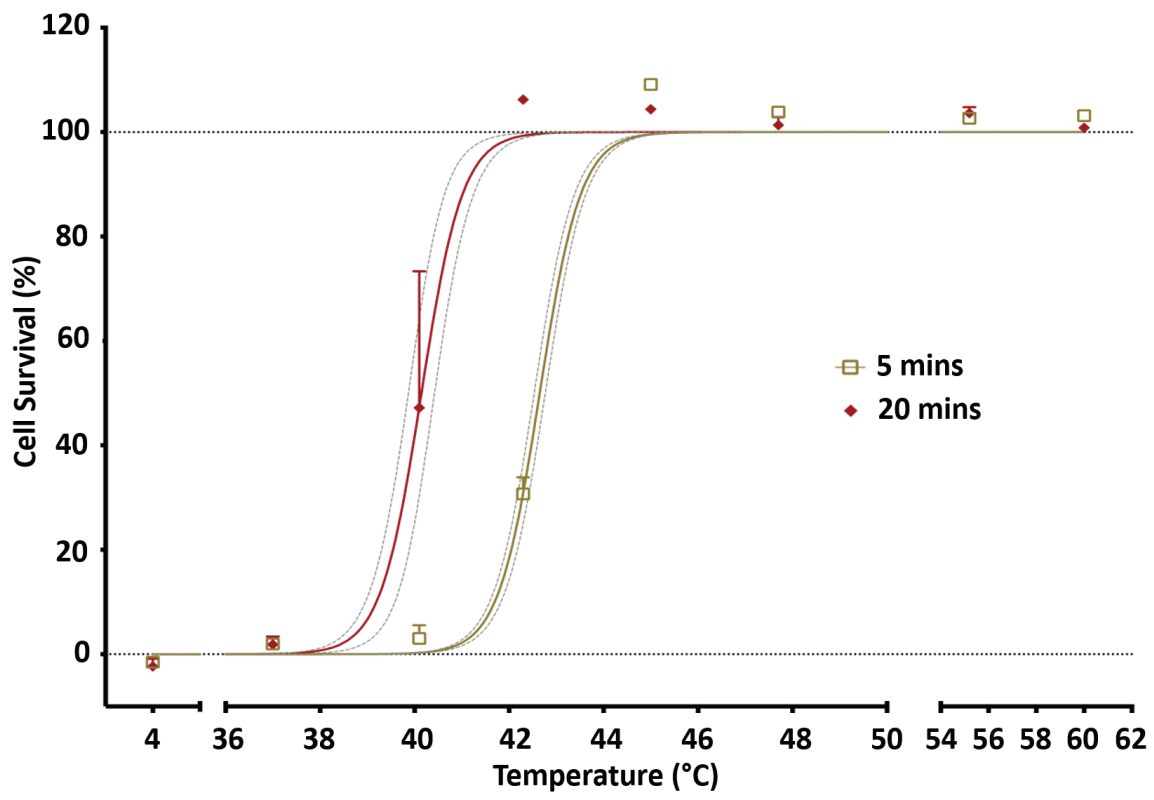


facilities (Figure 1). Venom was aspirated from the dorsal spine glands using a $29 \mathrm{~g}$ half-inch needle inserted through the skin membrane into the venom gland (Figure 2). Samples were frozen at $-80^{\circ} \mathrm{C}$, lyophilized and returned to a $-80^{\circ} \mathrm{C}$ freezer setting for storage until use. Venom was rehydrated with Dulbecco's phosphate buffered saline and centrifuged at $22,402 \mathrm{~g}$ for $2 \mathrm{~min}$. The centrifuged supernatant was cleaned using a $0.22 \mu \mathrm{m}$ filter and protein concentration determined using the Bradford Lowry protein assay. ${ }^{24}$

Subsequently, venom samples were heated to $4^{\circ} \mathrm{C}, 37.0^{\circ} \mathrm{C}$, $40.1^{\circ} \mathrm{C}, 42.3^{\circ} \mathrm{C}, 45.0^{\circ} \mathrm{C}, 47.7^{\circ} \mathrm{C}, 55.2^{\circ} \mathrm{C}$ and $60.0^{\circ} \mathrm{C}$ for incubation times of $5 \mathrm{~min}$ and $20 \mathrm{~min}$. Samples were then removed from the heating plate before being returned to an ice bath $\left(4.0^{\circ} \mathrm{C}\right)$ before testing. ACEA's xCELLigence system was used to assess the cytotoxicity of the venom on human cells in vitro. Samples were aliquoted into individual wells on the xCELLigence E-plate seeded with 5,000 human cardiomyocytes per well. A total of three replicates for each temperature and exposure time were used, with controls consisting of four replicates of unheated venom rehydrated with Dulbecco's phosphate buffered saline solution.

To understand the depth at which the integument sheath is broken and forces venom to be expelled through the spine, the first dorsal spine length was measured from spine tip to the beginning of the venom sac in individual animals. To achieve this measurement, a rubber sheet was compressed down onto the venom sac and the distance from the spine tip was recorded (Figure 3).

\section{STATISTICAL ANALYSIS}

Data were analyzed using an analysis of covariance for temperature as the independent variable, cell viability as the dependent variable and incubation time as the co-variate, with curves fitted using a variable hill slope (-1). The $0.01 \%$ inhibition concentration, Chi-square goodness of fit test (extra sum-of-squares F-test) and graphs were generated with GraphPad Prism v 6.0 (GraphPad Software Inc.).

\section{Results}

There was a significant difference between cell viability curves with incubation time (df $(1,44), F=146.70$, $P=<0.005$, Figure 4 ). The temperature range where time influenced activity was between 40 to $45^{\circ} \mathrm{C}$. Below $37^{\circ} \mathrm{C}$, both time points were insufficient to halt venom activity and above $48^{\circ} \mathrm{C}, 5 \mathrm{~min}$ incubation time was sufficient to destroy the venom activity. With $5 \mathrm{~min}$ incubation, the temperature required to neutralise venom from the $0.01 \%$ inhibitory concentration curve was $44.6^{\circ} \mathrm{C}(95 \%$ confidence limits of $44.5^{\circ} \mathrm{C}-44.8^{\circ} \mathrm{C}$ ). Incubating the venom for $20 \mathrm{~min}$ lowered the $0.01 \%$ inhibitory concentration temperature by approximately $2^{\circ} \mathrm{C}$, requiring only $42.1^{\circ} \mathrm{C}(95 \%$ confidence limits of $41.9^{\circ} \mathrm{C}-42.4^{\circ} \mathrm{C}$ ) to inactivate the venom.
The average depth in which venom was released from the spine upon compression of the venom sacs was approximately $18 \mathrm{~mm}$.

\section{Discussion}

Results demonstrate that exposure to heat significantly reduces the lethality of $S$. horrida venom through deactivation of venom components. More specifically, we demonstrated that exposure of $S$. horrida venom to temperatures above $39^{\circ} \mathrm{C}$ dramatically inhibits its cytotoxicity. This finding agrees with previous studies that suggest exposing venom to heat causes loss of its functionality and/or cytotoxicity. ${ }^{20,25}$ Moreover, this research shows that decreases in immersion temperature lead to longer incubation periods to render the venom biologically ineffective.

The finding that the average depth in which venom is released from the glands is approximately $18 \mathrm{~mm}$ from the spine tip is relevant to the first-aid for stonefish envenomation. An increased incubation time will increase the probability of heat penetrating deeper into tissues and deactivating the venom that has been deposited without damaging profounder tissue. When comparing this study to the current generalised first-aid management protocol, it is plausible to say that hot water immersion (HWI) therapy could possibly resolve stonefish venom intoxications in $20 \mathrm{~min}$ instead of the proposed $90 \mathrm{~min}$. Also, this can be achieved using a temperature at the lower range of the scale (i.e., $42^{\circ} \mathrm{C}$ ). This should also minimize the chance of the victim getting a skin burn from hotter temperatures. ${ }^{23}$

On the other hand, by subjecting the patient to a shorter incubation time, a higher temperature will be required. In this case, patients can be at a greater risk of suffering first aid complications (i.e., scalding injuries) caused by exposure to damaging temperatures around $46^{\circ} \mathrm{C} .{ }^{26}$ Also, some patients cannot tolerate high temperatures and discomfort can result. ${ }^{27}$ For these cases, further decreases in the HWI temperature could be helpful. Unfortunately, as this research only used two different incubation times, the time required to deactivate the venom at the $39^{\circ} \mathrm{C}$ threshold remains unknown. Moreover, further investigation using live animals is warranted to delineate both temperature and time thresholds in vivo.

\section{Conclusions}

An immersion time of $20 \mathrm{~min}$ at $42^{\circ} \mathrm{C}$ was sufficient to detoxify $S$. horrida venom in vitro. This could be recommended when clinicians select hot water immersion therapy to treat stonefish wounds. This procedure should maximise the successful deactivation of the toxin while minimizing the time in which the procedure is completed, diminishing the chances of the victim suffering secondary burns or discomfort. 


\section{References}

1 Russell FE. Weever fish sting: the last word. Br Med J (Clin Res Ed). 1983;287(6397):981-2.

2 Isbister GK. Venomous fish stings in tropical northern Australia. Am J Emerg Med. 2001;19:561-5.

3 Lee JYL, Teoh LC, Leo SPM. Stonefish envenomations of the hand - A local marine hazard: a series of eight cases and review of the literature. Ann Acad Med Singapore. 2004;33:515-20.

4 Sutherland SK, Tibballs J. Australian animal toxins: the creatures, their toxins, and care of the poisoned patient. Melbourne: Oxford University Press; 2001.

5 Church J, Hodgson W. The pharmacological activity of fish venoms. Toxicon. 2002;40:1083-93.

6 Williamson J, Fenner P, Burnett JW, Rifkin JF. Venomous and poisonous marine animals: a medical and biological handbook. Sydney: University of New South Wales Press; 1996.

7 Halstead BW, Courville DA. Poisonous and venomous marine animals of the world. Washington, DC: United States Government Printing Office; 1965.

8 Halstead BW, Chitwood MJ, Modglin FR. Stonefish stings, and the venom apparatus of Synanceja horrida (Linnaeus). Trans Am Microsc Soc. 1956;75:381-97.

9 Phoon WO, Alfred ER. A study of stonefish (Synanceja) stings in Singapore with a review of the venomous fishes of Malaysia. Singapore Med J. 1965;5:158.

10 Saunders P, Rothman S, Medrano V, Chin H. Cardiovascular actions of venom of the stonefish Synanceja horrida. Am J Physiol (Legacy Content). 1962;203:429.

11 Auerbach, PS. Marine envenomations. $N$ Engl J Med. 1991;325:486-93.

12 Smith JLB. Two rapid fatalities from stonefish stabs. Copeia. 1957;249.

13 Austin L, Gillis RG, Youatt G. Stonefish venom: some biochemical and chemical observation. Aust J Exp Biol Med Sci. 1965;43:79.

14 Deakins DE, Saunders PR. Purification of the lethal fraction of the venom of the stonefish Synanceja horrida (Linnaeus). Toxicon. 1967;4:257-60.

15 Garnier P, Goudeyperriere F, Breton P, Dewulf C, Petek F, Perriere C. Enzymatic properties of the stonefish (Synanceia verrucosa Bloch and Schneider, 1801) venom and purification of a lethal, hypotensive and cytolytic factor. Toxicon. 1995;33:143-55.
16 Kreger AS. Detection of a cytolytic toxin in the venom of the stonefish (Synanceia trachynis). Toxicon. 1991;29:733-43.

17 Poh CH, Yuen R, Khoo HE, Chung M, Gwee M, Gopalakrishnakone P. Purification and partial characterization of stonustoxin (lethal factor) from Synanceja horrida venom. Comp Biochem Physiol Part B. 1991;99:793-8.

18 Saunders P. Venom of the stonefish Synanceja horrida. Arch Int Pharmacodyn Ther. 1959;123:195-203.

19 Saunders P, Tökés L. Purification and properties of the lethal fraction of the venom of the stonefish Synanceja horrida (Linnaeus). Biochimica et Biophysica Acta. 1961;52:527-32.

20 Weiner S. Observations on the venom of the stonefish (Synanceja trachynis). Med J Aust. 1959;46:620-7.

21 Tay TKW, Chan HZ, Ahmad TST, Teh KK, Low TH, Wahab AN. Stonefish envenomation of hand with impending compartment syndrome. J Occup Med Toxicol. 2016;11:23.

22 Atkinson, PRT, Boyle A, Hartin D, McAuley D. Is hot water immersion an effective treatment for marine envenomation? Emerg Med J. 2006; 23:503-8.

23 Fenner P. Marine envenomation: an update - a presentation on the current status of marine envenomation first aid and medical treatments. Emerg Med. 2000;12:295-302.

24 Bradford MM. A rapid and sensitive method for the quantitation of microgram quantities of protein utilizing the principle of protein-dye binding. Anal Biochem. 1976;72:24854.

25 Carrette TJ, Cullen P, Little M, Peiera PL, Seymour JE. Temperature effects on box jellyfish venom: a possible treatment for envenomed patients? Med J Aust. 2002;177:654-5.

26 Moritz AR, Henriques FC. Studies of thermal injury: II. The relative importance of time and surface temperature in the causation of cutaneous burns. Am J Pathol. 1947;23:695-720.

27 Perkins RA, Morgan SS. Poisoning, envenomation and trauma from marine creatures. Am Fam Physician. 2004;69:885-90 +893 .

Submitted: 11 April 2017; revised 06 June 2017

Accepted: 28 June 2017

\section{Funding}

This study was internally funded by James Cook University.

Conflicts of interest: nil 


\title{
Decompressing rescue personnel during Australian submarine rescue operations
}

\author{
Michael P Reid ${ }^{1}$, Andrew Fock², David J Doolette ${ }^{3}$ \\ ${ }^{1}$ Submarine Underwater Medicine Unit, Royal Australian Navy, Sydney, Australia \\ ${ }^{2}$ Hyperbaric Unit, The Alfred Hospital, Melbourne, Australia \\ ${ }^{3}$ Navy Experimental Diving Unit, United States Navy, Panama City, USA
}

Corresponding author: Michael Reid, John Hunter Hospital, Department of Anaesthesia, Locked Bag 1, Hunter Region Mail Centre, NSW, 2310, Australia

mreid11@me.com

\section{Key words}

Decompression tables; Decompression sickness; Probability; Models; Oxygen; Military diving; Environmental medicine

\begin{abstract}
(Reid MP, Fock A, Doolette DJ. Decompressing rescue personnel during Australian submarine rescue operations. Diving Hyperbaric Medicine. 2017 September;47(3):159-167.)

Introduction: Personnel rescuing survivors from a pressurized, distressed Royal Australian Navy (RAN) submarine may themselves accumulate a decompression obligation, which may exceed the bottom time limits of the Defense and Civil Institute of Environmental Medicine (DCIEM) Air and In-Water Oxygen Decompression tables (DCIEM Table 1 and 2) presently used by the RAN. This study compared DCIEM Table 2 with alternative decompression tables with longer bottom times: United States Navy XVALSS_DISSUB 7, VVAL-18M and Royal Navy 14 Modified tables.

Methods: Estimated probability of decompression sickness $\left(\mathrm{P}_{\mathrm{DCS}}\right)$, the units pulmonary oxygen toxicity dose (UPTD), the volume of oxygen required and the total decompression time were calculated for hypothetical single and repetitive exposures to $253 \mathrm{kPa}$ air pressure for various bottom times and prescribed decompression schedules.

Results: Compared to DCIEM Table 2, XVALSS_DISSUB 7 single and repetitive schedules had lower estimated $\mathrm{P}_{\mathrm{DCS}}$, which came at the cost of longer oxygen decompressions. For single exposures, DCIEM schedules had $\mathrm{P}_{\mathrm{DCS}}$ estimates ranging from $1.8 \%$ to $6.4 \%$ with 0 to 101 UPTD and XVALSS_DISSUB 7 schedules had $\mathrm{P}_{\mathrm{DCS}}$ of less than 3.1\%, with 36 to 350 UPTD. Conclusions: The XVALSS_DISSUB 7 table was specifically designed for submarine rescue and, unlike DCIEM Table 2, has schedules for the estimated maximum required bottom times at $253 \mathrm{kPa}$. Adopting these tables may negate the requirement for saturation decompression of rescue personnel exceeding DCIEM limits.
\end{abstract}

\section{Introduction}

The ambient pressure inside a distressed submarine (DISSUB) may become elevated above $101 \mathrm{kPa}$ owing to compression of the remaining gas space by partial flooding or release of high-pressure gas supplies. ${ }^{1}$ Locating the DISSUB and delivering the rescue system to the site may take several days resulting in the crew of the DISSUB becoming saturated (inert gas tissue tensions equilibrate with the inspired inert gas pressures) at elevated pressure. A Submersible Rescue Vehicle (SRV) that can mate with the escape hatch of the DISSUB may rescue survivors (Figure 1). ${ }^{1,2}$ To accomplish the evacuation, ambient pressure inside the SRV must be equalized with the DISSUB internal pressure. ${ }^{1}$ The SRV remains pressurized at the DISSUB pressure during the transit to the surface where the survivors are again transferred under pressure to a recompression chamber (RCC) located on-board a rescue ship in order to complete saturation decompression. Rescue personnel who are exposed to the DISSUB pressure accrue their own decompression obligation.
The current Royal Australian Navy (RAN) DISSUB rescue system uses the James Fisher Defence 'LR5' SRV, which can rescue up to 14 seated, $80 \mathrm{~kg}$ survivors per sortie (excluding stretcher cases), a transfer under pressure (TUP) compartment, and two RCCs that can each accommodate seven survivors and one medical attendant (Figure 2). On the surface, the SRV mates to the TUP compartment and survivors are transferred, one at a time, between the TUP compartment and the RCCs using one-man, portable chambers (Figure 2). This process is labour intensive and susceptible to delays owing to inclement weather or the need to transfer immobilised patients. Rescue sorties are separated by a surface interval time (SIT) of many hours, as the SRV can only redeploy to the DISSUB two hours before the previous cohort of survivors are due to complete decompression so that the RCCs are available for the next cohort of survivors.

A Collins class submarine can accommodate 65 people, including crew and other personnel. It is estimated that six SRV sorties will be required to evacuate the DISSUB of rescue personnel and survivors, based on the average 
Figure 1

Overview of Royal Australian Navy submarine rescue operations

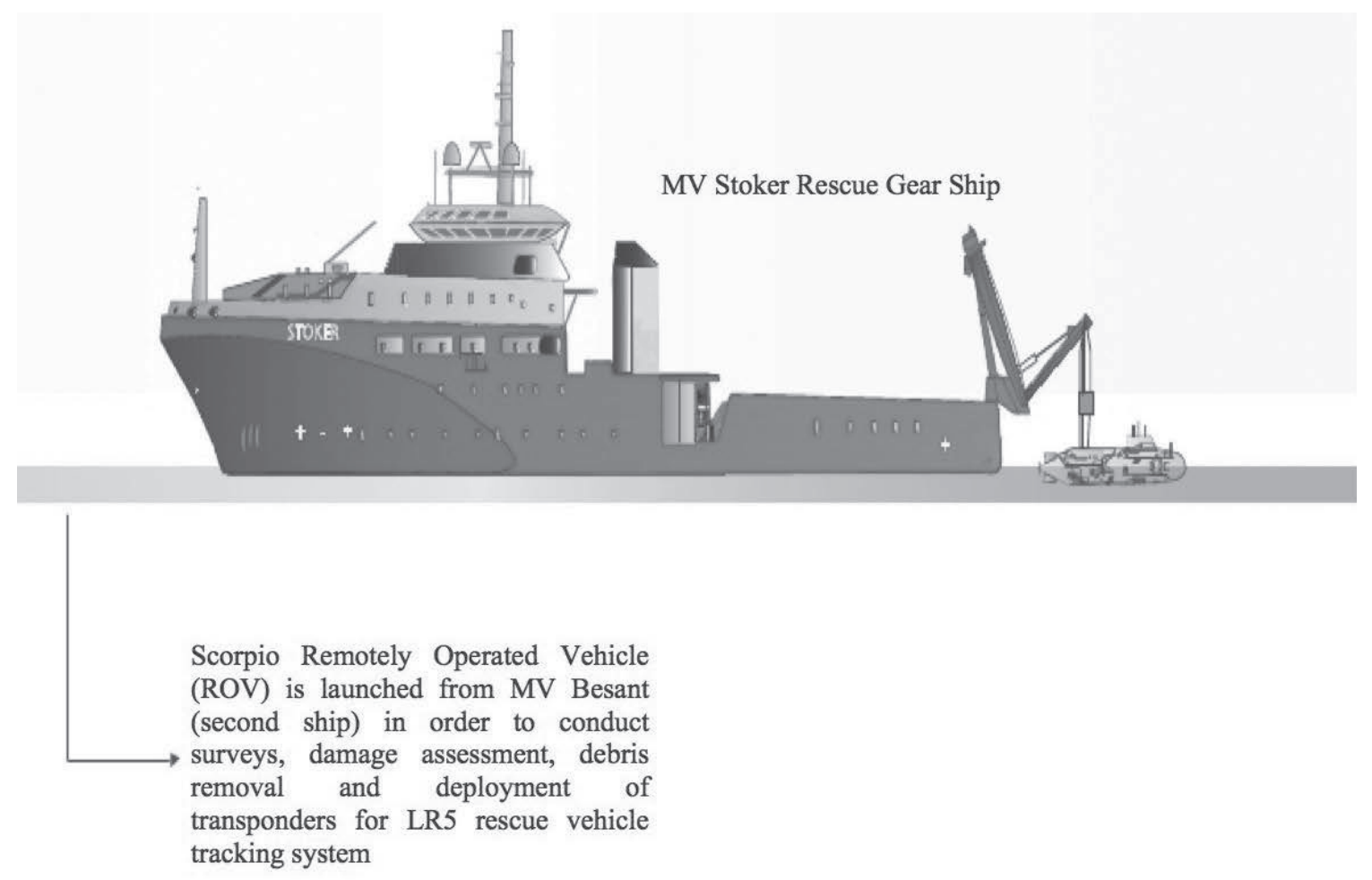

I.R5 Suhmersible Rescue Vehicle
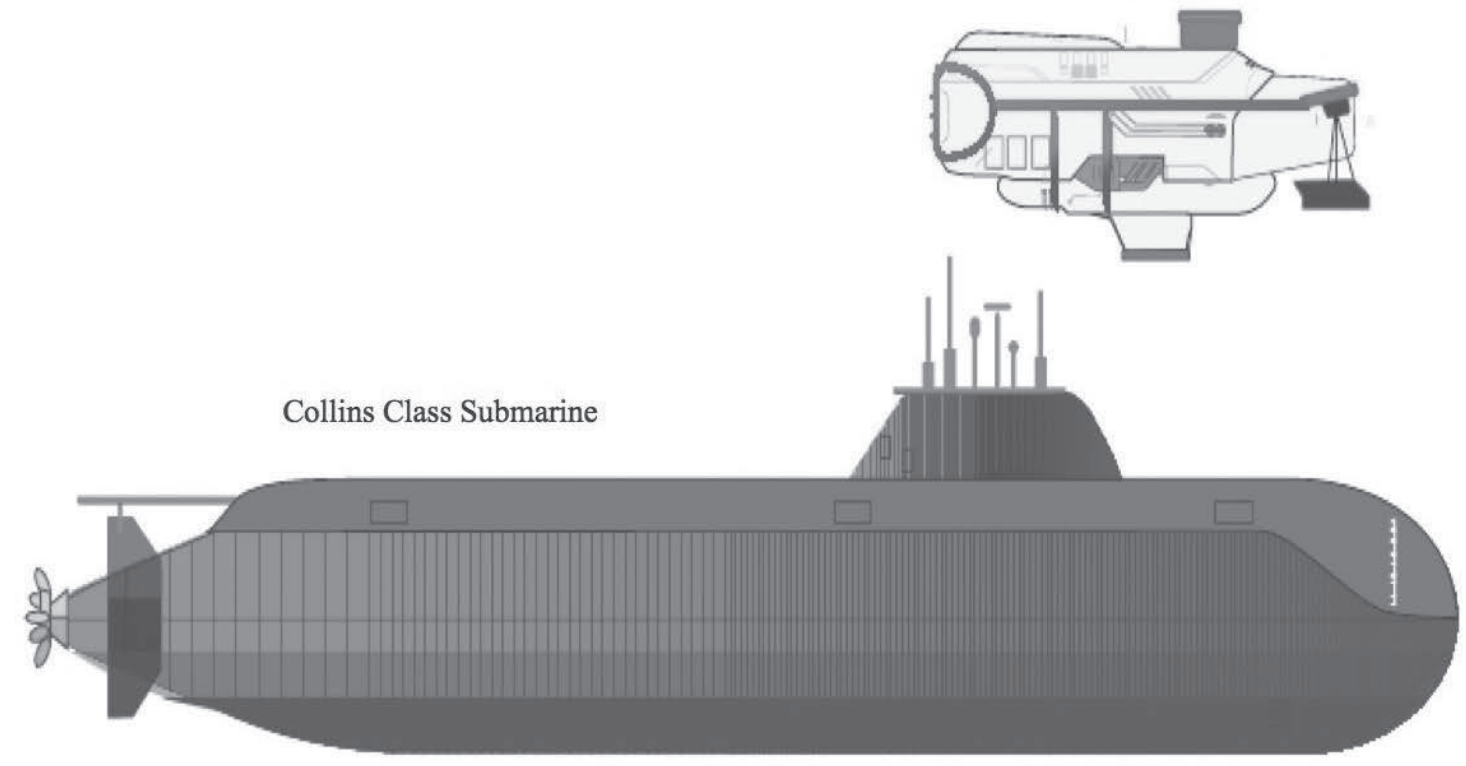

weight of a RAN submariner (96 kg) (Ponton, K, personal communication; 2013) and assuming all personnel can be seated. The number of sorties will increase if patients need to be immobilised on stretchers.

Pilots housed in the forward SRV compartment remain at $101 \mathrm{kPa}$ hence do not require decompression, but separate medical personnel attending survivors inside the aft section of the SRV, the TUP compartment, and the RRCs each accrue their own decompression obligation. Each SRV sortie requires approximately 240 minutes (min) and the medical attendant in the aft compartment of the SRV will be at the DISSUB pressure along with the rescued survivors for approximately $180 \mathrm{~min}$ after equalization with the DISSUB internal pressure and during the return to the rescue ship. The SRV aft compartment remains pressurized for a further 60-150 min to permit transfer of 14 survivors from one SRV sortie to the deck RCCs. An additional $15 \mathrm{~min}$ is required to 
Figure 2

Deck layout of the RAN submarine rescue system on-board the rescue vessel. The SRV (LR5) is in position to mate with the TUP compartment, which in turn can mate with three portable, one-man recompression chambers (OMRCs). OMRCs are manually wheeled across the deck to mate with the Type B RCCs
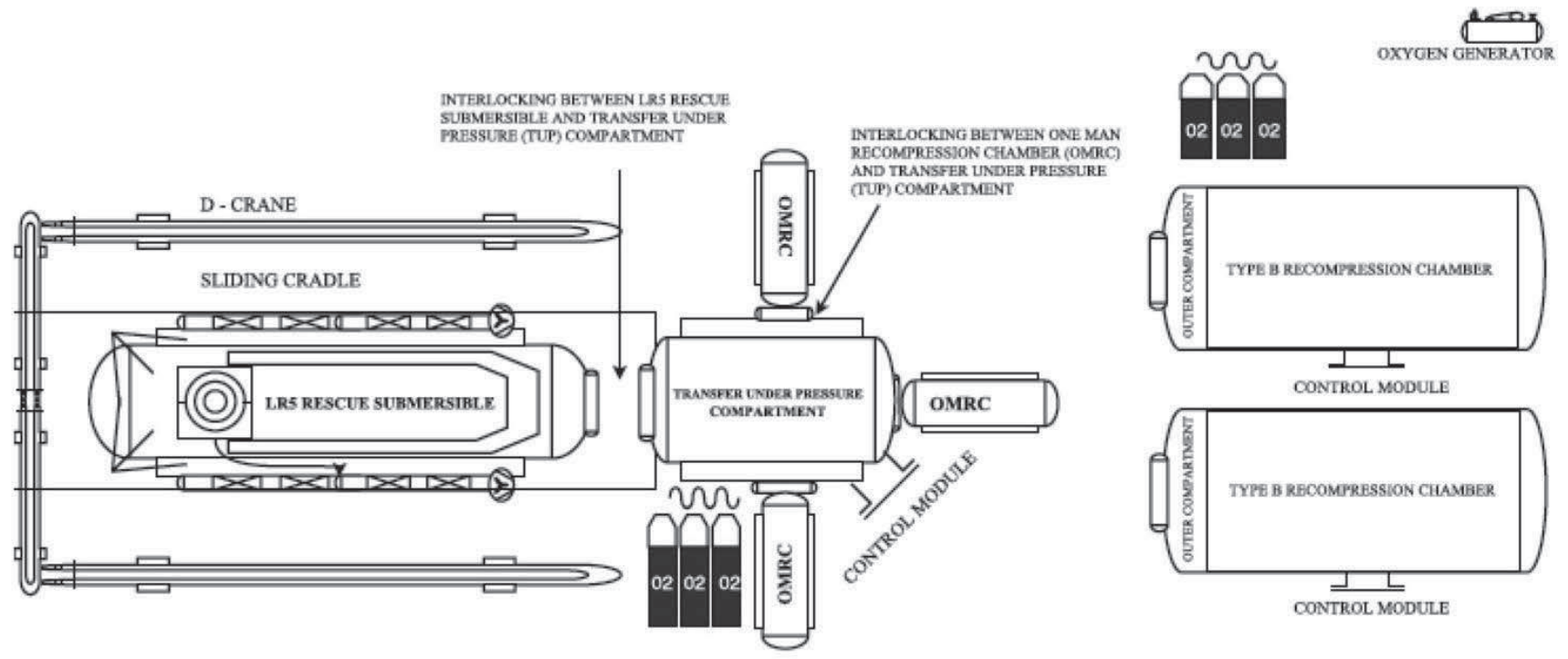

close the RCC inner lock and commence survivor saturation decompression. This frees the RCC outer lock for oxygen $\left(\mathrm{O}_{2}\right)$-accelerated decompression of medical attendants. Since neither the SRV nor the TUP compartments are equipped for $\mathrm{O}_{2}$-accelerated decompression, the SRV medical attendant transfers to the RCC outer lock for $\mathrm{O}_{2}$-accelerated decompression, whilst the TUP medical attendant undergoes air decompression in the TUP compartment.

RAN DISSUB planning assumes all souls could be rescued from a DISSUB pressure of $253 \mathrm{kPa}$. Higher DISSUB pressures are possible, but RAN analysis of Collins Class submarines has assessed that likely conditions associated with such pressures are not survivable, and would require the crew to escape rather than await rescue (classified or restricted access). ${ }^{3-5}$ At present, the only decompression tables authorized for decompression of RAN medical attendants are the Defense and Civil Institute of Environmental Medicine Air and In-Water $\mathrm{O}_{2}$ schedules (DCIEM Tables 1 and 2 respectively). ${ }^{6,7}$ Both tables are designed for underwater diving operations and have a maximum bottom time at $253 \mathrm{kPa}$ of $280 \mathrm{~min},{ }^{7}$ insufficient to accommodate the 345 min exposure possible for the SRV medical attendant.

Fresh medical attendants could lock-in before the limits of DCIEM tables are exceeded; however, there may not always be sufficient personnel for the longest exposures. Current RAN planning requires medical attendants who have exceeded the limits of the DCIEM tables to be decompressed on the same saturation schedule as survivors (Hissink J, personal communication, 2013).

The National Oceanic and Atmospheric Administration
(NOAA) 17.11 standard $\mathrm{O}_{2}$-accelerated saturation decompression table is currently favoured for saturation decompression of survivors owing to its relatively short total decompression time of 680 min from $253 \mathrm{kPa}{ }^{8}$ Nevertheless, saturation decompression of medical attendants from exposures of $345 \mathrm{~min}$ or less is unnecessary and costly in terms of $\mathrm{O}_{2}$ supply, $\mathrm{RCC}$ space, and human resources on-board the rescue ship.

As a result of these constraints, the first author was tasked by the Officer-in-Charge, Submarine Underwater Medicine Unit to investigate alternative decompression tables that would permit decompression of personnel with bottom times up to $345 \mathrm{~min}$ at $253 \mathrm{kPa}$. VVAL-18M is the algorithm underlying the air decompression tables in the United States Navy (USN) Diving Manual, Revision 6, and is intended for diving operations, but has schedules for bottom times up to 420 min at $253 \mathrm{kPa}^{9}{ }^{910}$ The Royal Navy Table 14 (RN14) was originally a diving table, retrospectively modified for submarine rescue, and has schedules for bottom times up to $350 \mathrm{~min}$ at $253 \mathrm{kPa} .{ }^{11,12}$ The USN XVALSS_DISSUB 7 Table were specifically designed for submarine rescue and has schedules for bottom times up to $460 \mathrm{~min}$ at $253 \mathrm{kPa} .^{13}$

To evaluate the utility of these alternative tables, this paper compares the estimated probability of decompression sickness $\left(\mathrm{P}_{\mathrm{DCS}}\right)$, the units pulmonary toxicity dose (UPTD), volume of oxygen required and the total dive time (TDT) of single and repetitive exposures to $253 \mathrm{kPa}$ followed by $\mathrm{O}_{2}-$ accelerated decompression prescribed by DCIEM, VVAL18M, XVALSS DISSUB 7, and RN14-Modified tables for exposures relevant to SRV and TUP medical attendants. ${ }^{6,7,9-13}$ A companion paper evaluates strategies for decompressing RCC medical attendants. ${ }^{14}$ 


\section{Methods}

The approach was to analyse hypothetical dive profiles (pressure/time/breathing gas histories) representing single or repetitive exposures to a DISSUB ambient pressure of $253 \mathrm{kPa}$ for various bottom times, and followed by decompression prescribed by each of the four candidate decompression tables that had a schedule for that exposure. In all these profiles, an $85 \%$ inspired oxygen fraction $\left(\mathrm{F}_{\mathrm{i}} \mathrm{O}_{2}\right)$ was assumed during $\mathrm{O}_{2}$ breathing, to account for leakage of chamber atmosphere into a demand-valve oral nasal mask. ${ }^{15}$ If higher $\mathrm{FiO}_{2}$ can be achieved, the actual $\mathrm{P}_{\text {DCS }}$ will be lower than estimated.

Single dive bottom times ranged from 60 to $460 \mathrm{~min}$ followed by decompression prescribed by the DCIEM InWater $\mathrm{O}_{2}$ Decompression Table (DCIEM Table 2, for dives up to $280 \mathrm{~min}$ bottom time), ${ }^{7} \mathrm{RN} 14-$ Modified (for dives up 350 min bottom time), ${ }^{11,12}$ VVAL-18M In-Water $\mathrm{O}_{2}$ Decompression tables (for dives up 420 min bottom time), ${ }^{9,10}$ and XVALSS_DISSUB 7 Table (for all dives). ${ }^{13}$ Repetitive exposures comprised two identical bottom times of 60 and $150 \mathrm{~min}$ at $253 \mathrm{kPa}$ separated by a surface interval time (SIT) of 12.5 hours (h), with decompression from each dive as prescribed by the candidate tables, except for the RN14Modified tables that do not permit repetitive diving. ${ }^{11,12}$

The 12.5-h SIT was in accord with the time between SRV sorties if survivors must be decompressed from $253 \mathrm{kPa}$ saturation. In addition to examining dive profiles representing the full bottom time of schedules, some dive profiles representing likely medical attendant exposures with decompression schedules selected using standard round-up conventions were also examined. In all, 160 single and 12 repetitive dive profiles were examined.

The $\mathrm{P}_{\mathrm{DCS}}$ for each complete dive profile was calculated using the Navy Medical Research Institute 98, model 2 (NMRI98) and Bubble Volume Model 3 [BVM(3)] probabilistic models for DCS incidence and time of occurrence. ${ }^{16,17}$ Each of these models uses a dive profile as input and calculates the theoretical time course of gas partial pressures in each of three well-stirred compartments with different half-times. In the NMRI-98 model, the hazard (instantaneous risk) is the sum across compartments of positive values of functions of the gas supersaturation. The $\mathrm{P}_{\mathrm{DCS}}$ is a function of the timeintegral of the hazard from the beginning of a dive profile until the point in time long after the dive when the hazard finally declines to zero. ${ }^{16}$

For repetitive dives, the cumulative $\mathrm{P}_{\mathrm{DCS}}$ is the sum of the risks of all dives performed and the gas pressures are tracked throughout the repetitive dives and intervening SIT. BVM(3) includes a single bubble in each compartment and the hazard is a function of the calculated bubble volume in the three modelled tissue compartments. ${ }^{17}$ The parameters that govern the gas kinetics and bubble dynamics of these models (for instance compartment half-times and gas diffusivities) are selected by a best practicable fit to a large, diverse database of dive profiles (approximately $5 \%$ incidence of DCS) from carefully controlled and monitored air and nitrox man dives. ${ }^{16,17}$ These two models are fit to similar data sets. Widely dissimilar estimates between these two structurally different models would be considered evidence of inappropriate extrapolation to dives unlike the calibration data.

The oxygen consumption, UPTD, and TDT were calculated for each of these dive profiles. The UPTD concept is based on inspired $\mathrm{PO}_{2}$ and exposure time ( $\mathrm{t}$ ) isopleths for equivalent decrements in vital capacity (as a marker for pulmonary oxygen toxicity). The UPTD is the exposure time in minutes at 1 atmosphere absolute inspired $\mathrm{PO}_{2}$ required to produce the equivalent pulmonary oxygen toxicity to any arbitrary $\mathrm{PO}_{2}$ time exposure. ${ }^{18}$ The original UPTD was based on the equation:

$$
\left(\mathrm{PO}_{2}-0.5\right) t^{1.2}=c
$$

Instead we used the alternative equation:

$$
-0.011\left(\mathrm{PO}_{2}-0.5\right) t=c
$$

This results in:

$$
\text { UPTD }=t\left(\frac{\mathrm{PO}_{2}-0.5}{0.5}\right)
$$

which is of similar form to the original UPTD derivation. ${ }^{18,19}$ We provide UPTD to compare the oxygen exposure of the different profiles without comment on the estimated percentage decrement in vital capacity. Furthermore, cumulative UPTD for repetitive dives does not account for recovery from air breathing.

Oxygen consumption was based on a conservative respiratory minute ventilation of $15 \mathrm{~L} \cdot \mathrm{min}^{-1}$, adjusted for Boyle's Law and at body temperature and pressure, saturated (BTPS). Fifteen $\mathrm{L} \cdot \mathrm{min}^{-1}$ was based on a tidal volume of 10 $\mathrm{mL} \cdot \mathrm{kg}^{-1}$, a resting adult respiratory rate of 15 breaths $\cdot \mathrm{min}^{-1}$ and a body weight of $100 \mathrm{~kg}$ (the latter based on the 95th percentile for weight in Australian submariners being 96 $\mathrm{kg}){ }^{20}$ TDT was calculated using recommended air-breaks. VVAL-18M and RN14-Modified tables recommend a 5-min air-break after every 30 min of oxygen breathing, ${ }^{9-12}$ whereas XVALSS_DISSUB 7 tables require a 15-min air-break after every 60 min of oxygen breathing. ${ }^{13}$ DCIEM Table 2 does not require air-breaks. ${ }^{7}$

\section{Results}

\section{SINGLE DIVES}

Figures 3 and 4 give the BVM(3)-estimated $\mathrm{P}_{\mathrm{DCS}}$ for DCIEM 2, VVAL-18M, XVALSS_DISSUB 7 and RN14-Modified decompression following single dives at a depth of 253 $\mathrm{kPa}$ for the full bottom time of the published schedules. NMRI-98-estimated $\mathrm{P}_{\text {DCS }}$ were not substantially different for any of the dive profiles evaluated, providing confidence that both models were used in their reliable range. Owing to differences in table increments and limits, a direct comparison could not be made between all dive profiles. 
Figure 3

$\mathrm{P}_{\mathrm{DCS}}(\%, \mathrm{y}$-axis) in rescue personnel for single dives with bottom times from 60-280 min (x-axis) at $253 \mathrm{kPa}$ estimated using the BVM(3) model; at each bottom time the cluster of bars gives the $\mathrm{P}_{\mathrm{DCS}}$ for decompression according to each table that has a schedule for that bottom time; the order in the bar cluster is always the same but not all bars may appear

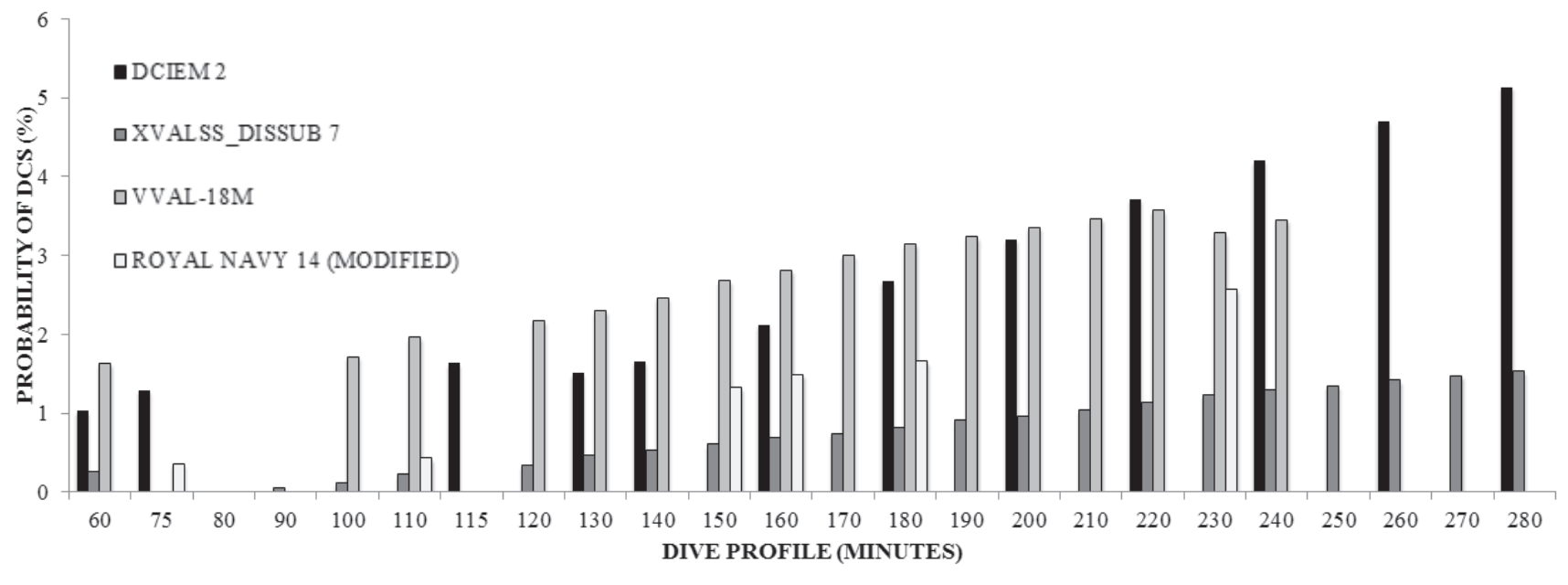

Figure 4

$\mathrm{P}_{\mathrm{DCS}}(\%, \mathrm{y}$-axis) in rescue personnel for single dives with bottom times from 290-460 min (x-axis) at $253 \mathrm{kPa}$ estimated using the $\mathrm{BVM}(3)$ model; at each bottom time the cluster of bars gives the $\mathrm{P}_{\mathrm{DCS}}$ for decompression according to each table that has a schedule for that bottom time; the order in the bar cluster is always the same but not all bars may appear

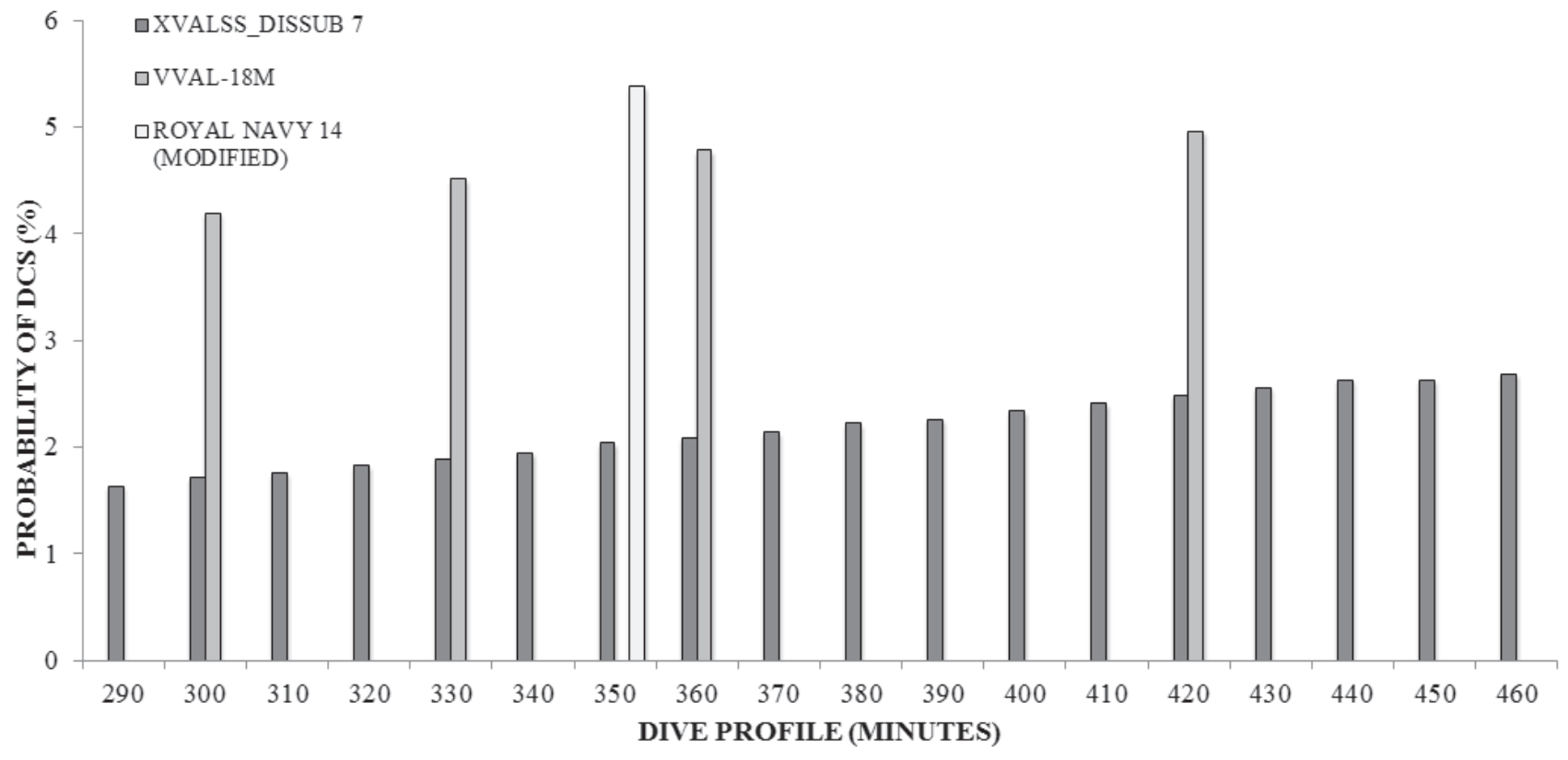

All decompression tables provided reasonably low risk decompression for single dives, with few profiles exceeding $5 \% \mathrm{P}_{\mathrm{DCS}}$. For single dive profiles XVALSS_DISSUB 7 tables produced the lowest $\mathrm{P}_{\mathrm{DCS}}$ risk estimates, whereas DCIEM Table 2 produced the highest.

The hyperbaric exposures required of the medical attendant in the SRV aft compartment are too long to allow for repetitive diving and have to be undertaken as single dives by 'clean' personnel (who have not had an hyperbaric exposure in the preceding $18 \mathrm{~h}$ ). However, medical attendants inside the TUP compartment may have exposures of $150 \mathrm{~min}$ or less, which could be undertaken as a repetitive exposure after the 12.5-h SIT imposed by the turn-around time for SRV sorties.

\section{REPETITIVE DIVES}

The $\mathrm{P}_{\mathrm{DCS}}$ of repetitive dives can be increased compared to single dives because of residual inert gas or bubbles from the preceding dive. The BVM(3)-estimated cumulative $\mathrm{P}_{\mathrm{DCS}}$ for repetitive 60 -min dives at $253 \mathrm{kPa}$ with a SIT of 
Figure 5

Unit pulmonary toxicity dose (UPTI) in rescue personnel for single dives with bottom times from $75-460 \mathrm{~min}$ at $253 \mathrm{kPa}$; at each bottom time the cluster of bars gives the UPTD for decompression according to each table that has a schedule for that bottom time; the order in the bar cluster is always the same but not all bars may appear

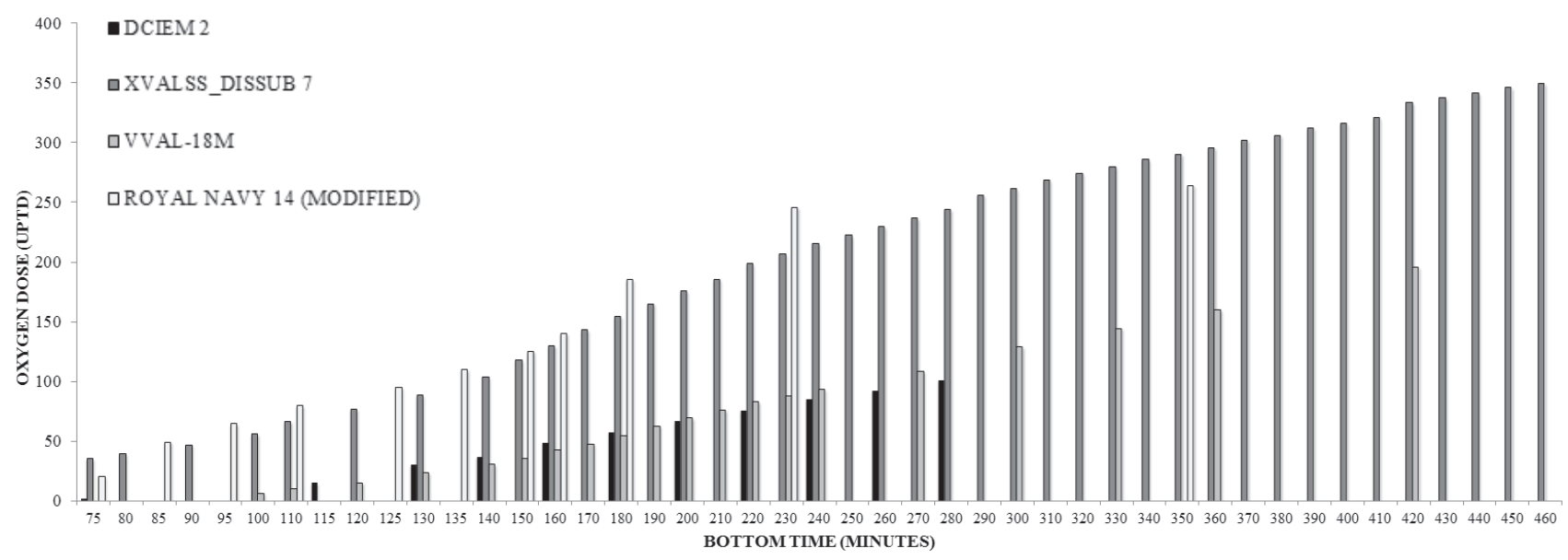

$12.5 \mathrm{~h}$ using DCIEM 2 was $2.1 \%$, using VVAL-18M was $2.0 \%$, and for XVALSS_DISSUB 7 the cumulative $\mathrm{P}_{\mathrm{DCS}}$ was $0.6 \%$. Likewise, the NMRI-98-estimated cumulative $\mathrm{P}_{\mathrm{DCS}}$ for repetitive 60-min dives at $253 \mathrm{kPa}$ with a SIT of $12.5 \mathrm{~h}$ using DCIEM 2 was $2.9 \%$, using VVAL-18M was $2.8 \%$ and for XVALSS_DISSUB 7 was $1.1 \%$.

Repetitive 150-min dives with a 12.5-h SIT decompressed using VVAL-18M tables have a cumulative BVM(3)estimated $\mathrm{P}_{\text {DCS }}$ of $5.6 \%$. Cumulative NMRI-98-estimated $\mathrm{P}_{\text {DCS }}$ for VVAL-18M tables was 6.0\%. DCIEM 2 and XVALSS_DISSUB 7 tables do not permit a repetitive 150 -min dive at $253 \mathrm{kPa}$ with a SIT of 12.5 hours. ${ }^{7,13}$ The RN14-Modified table does not support any repetitive diving. ${ }^{11,12}$

The cumulative $\mathrm{P}_{\text {DCS }}$ for these repetitive dives gives a sense of the overall risk to an individual TUP operator. However, another consideration is the probability of any DCS occurring in the rescue cohort, even if conducting single-dives. Depending on which decompression tables are selected, between 12 to 18 SRV/TUP hyperbaric personnel-exposures will be required to rescue 65 survivors. The probability of a single DCS incident in the course of a diving operation is greater than the $\mathrm{P}_{\mathrm{DCS}}$ of a single or repetitive dive. The probability of at least one incidence of DCS in a series of identical dives can be determined using binomial theorem, and is one minus the probability of no DCS in all dives.

For illustrative purposes, consider that all rescue sorties were for a $345-\mathrm{min}$ bottom time at $253 \mathrm{kPa}$. For decompression according to the closest VVAL-18M schedule (360 min), which results in a $\mathrm{BVM}(3)$-estimated $\mathrm{P}_{\mathrm{DCS}}$ of $4.8 \%$, the probability of at least one case of DCS among all SRV and TUP medical attendants performing 18 hyperbaric exposures is $1-(1-0.048)^{18}=58.7 \%$. If instead decompression is conducted according to the closest XVALSS_DISSUB 7 schedule (350 minutes), which has a BVM(3)-estimated $\mathrm{P}_{\mathrm{DCS}}$ of $2.0 \%$, the probability of at least one case of DCS among all SRV and TUP medical attendants is $1-(1-0.02)^{18}=30.4 \%$.

\section{OXYGEN EXPOSURE AND REQUIREMENTS}

Figure 5 summarizes the UPTD units for single dive profiles at $253 \mathrm{kPa}$ following decompression with each of the XVALSS_DISSUB 7, DCIEM 2, RN14-Modified and VVAL-18M tables. XVALSS_DISSUB 7 tables delivered the highest UPTD, whereas DCIEM Table 2 delivered the lowest. No UPTD exceeded repetitive excursion (REPEX) recommendations for daily UPTD dose limits. ${ }^{21}$ For example, medical attendants performing a single dive are permitted a daily and total cumulative dose of 850 units, whereas personnel performing two dives during the rescue operation are restricted to a daily and total pulmonary toxicity dose of 700 and 1400 units respectively. ${ }^{21}$

XVALSS_DISSUB 7 and RN14-Modified had higher oxygen requirements in comparison to VVAL-18M and DCIEM Table 2. For the longest predicted bottom time of $345 \mathrm{~min}$ (at $253 \mathrm{kPa}$ and adjusted for Boyles Law/BTPS) XVALSS_DISSUB 7, VVAL-18M and RN14-Modified required 4689, 2221 and 3033 litres respectively. Figure 6 compares TDTs for DCIEM 2, VVAL-18M, XVALSS DISSUB 7 and RN14-Modified tables. XVALSS_DISSUB 7 tables had the longest TDTs whereas DCIEM Table 2 had the shortest.

\section{Discussion}

The NMRI-98 and BVM(3) probabilistic decompression models were selected to evaluate candidate decompression 
Figure 6

Total decompression time ( $\mathrm{min}$ ) in rescue personnel for single dives with bottom times from $75-460 \mathrm{~min}$ at $253 \mathrm{kPa}$, calculated using recommended, minimum air-breaks; at each bottom time, the cluster of bars gives the total decompression time for each table that has a schedule for that bottom time; the order in the bar cluster is always the same but not all bars may appear

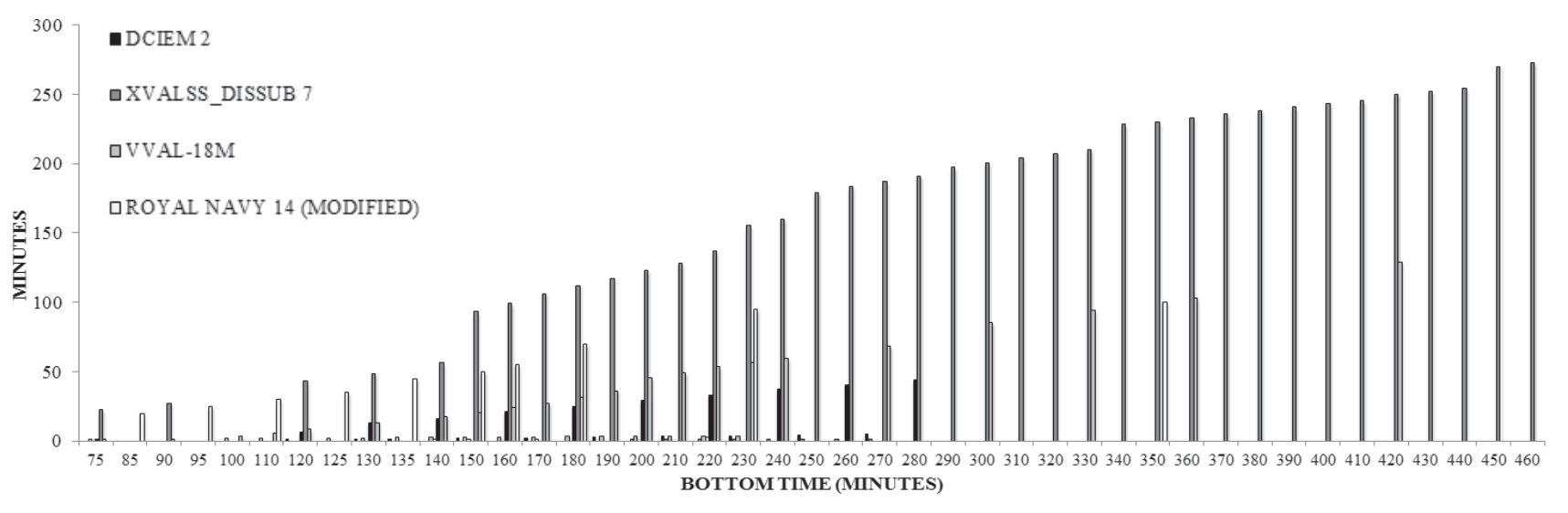

tables for use in DISSUB rescue operations. Both of these models were used in the original development and evaluation of the XVALSS_DISSUB 7 tables. ${ }^{13}$ These tables were calculated using the deterministic Thalmann algorithm, but with a parameter set (XVALSS_DISSUB 7) developed to produce schedules with a low NMRI-98 estimated $\mathrm{P}_{\mathrm{DCS}}$. The man-tested schedules were evaluated with both NMRI-98 and BVM(3). ${ }^{13}$ The present work differs in that it evaluates schedules relevant to a RAN DISSUB scenario. It must be acknowledged that the results presented are modelestimated $\mathrm{P}_{\mathrm{DCS}}$ and not the result of actual man-trials of the schedules likely in the RAN DISSUB scenario. However, these probabilistic models have been used extensively and provide credible estimates of $\mathrm{P}_{\mathrm{DCS}}$. A similar estimate from the two structurally different models provides additional confidence in the results.

The RAN does not have a policy on acceptable $\mathrm{P}_{\mathrm{DCS}}$ for diving or DISSUB rescue operations. The DCIEM Table 2 , which is approved for RAN use, had DCS incidence of $3.2-3.5 \%$ during development and validation. ${ }^{22-24}$ Most US Navy air and nitrox decompression procedures have an upper limit of 5\% $\mathrm{P}_{\mathrm{DCS}}$ for normal exposure diving. ${ }^{25}$ Severe central nervous system DCS is uncommon in air dives with less than about $7 \%$ estimated $\mathrm{P}_{\mathrm{DCs}}{ }^{26}$ These figures provide some objective criteria for evaluation of $\mathrm{P}_{\mathrm{DCS}}$ estimates. However, any DCS in medical attendants will result in serious strain on resources and, therefore, the lowest practicable $\mathrm{P}_{\mathrm{DCS}}$ is desirable. Nevertheless, low $\mathrm{P}_{\mathrm{DCS}}$ must be balanced against TDT, UPTD and oxygen use.

For single dives, the major advantage of using XVALSS DISSUB 7, VVAL-18M and RN14-Modified over DCIEM Table 2 is their longer table limits, ${ }^{7,9-13}$ which covers the worst case, 345-min dive profile for SRV medical attendant and, therefore, obviates the need for these attendants to undergo saturation decompression with the survivors. For single dives XVALSS_DISSUB 7 tables provided the lowest $\mathrm{P}_{\mathrm{DCS}}$ for dive profiles up to $420 \mathrm{~min}$ at $253 \mathrm{kPa}$ (Figures 3 and 4).

The advantages of these lower $\mathrm{P}_{\mathrm{DCS}}$ become particularly evident when assessing the probability of having at least one DCS among the medical attendants in the course of the rescue operation. It was specifically to mitigate this risk of DCS over the course of multiple dives during submarine rescue, that the XVASS_DISSUB 7 Table was developed. Human validation trials of XVALSS_DISSUB 7 for repetitive diving were tested under dry hyperbaric conditions relevant to submarine rescue personnel. Eight two-dive, repetitive profiles at depths between 193 to $284 \mathrm{kPa}$, were tested with a total of three cases of DCS during 125 dives. ${ }^{13}$

One DCS case occurred after each of the three repetitive $284 \mathrm{kPa}$ schedules: two 180-min exposures separated by a 56-minute SIT; two 240-min exposures separated by a 66min SIT and two 360-min exposures separated by a 126-min SIT. ${ }^{13}$ It is notable that these DCS occurred after repetitive dives to greater pressure and with shorter SIT than the RAN requirement and it is possible that a lower incidence of DCS would result in the RAN DISSUB scenario.

The risks of oxygen toxicity with these procedures are acceptable as no estimated UPTD exceeded REPEX recommendations. ${ }^{21}$ The risk of central nervous system (CNS) oxygen toxicity is considered low as the deepest decompression stops for RN14-Modified, XVALSS DISSUB 7, DCIEM 2 and VVAL-18M tables are 243, 223, 193 and $162 \mathrm{kPa}$ respectively ${ }^{7,9-13}$ and within a dry environment, the risk of oxygen toxicity seizures ranges from $1: 1,000$ to $1: 50,000 .{ }^{27}$

The lower oxygen requirements and shorter TDT for the DCIEM Table 2 and VVAL-18M Table justifies their retention and/or introduction for Australian submarine rescue (Figure 6). Although decompressing personnel with 
either DCIEM Table 2 or VVAL-18M tables carries a higher risk of DCS, there may be exceptional circumstances such as severe limitation of oxygen supply or time constraints where one must tolerate higher DCS risks in order to preserve oxygen for patients or expedite the rescue.

\section{Conclusion}

The introduction of candidate tables will enable decompression of medical attendants with bottom times up to $460 \mathrm{~min}$ at $253 \mathrm{kPa}$, thus negating the previous RAN requirement to decompress personnel exceeding the 280-min DCIEM Table 2 limit (at $253 \mathrm{kPa}$ ) with schedules designed for saturation divers. The XVALSS_DISSUB 7 Table provides acceptable $\mathrm{P}_{\mathrm{DCS}}$ limits of less than $3.1 \%$ for single dive profiles up to $460 \mathrm{~min}$ at $253 \mathrm{kPa}$. The VVAL-18M Table allows decompression up to 420 -min at $253 \mathrm{kPa}$ with shorter TDT and lower oxygen requirements, albeit with higher $\mathrm{P}_{\text {DCS }}$ estimates (up to 5.9\%). The RN14-Modified Table has little utility for Australian submarine rescue owing to an inability to plan repetitive diving.

\section{References}

1 Eckenhoff RG. Pressurized submarine rescue. Groton (CT): Navy Submarine Medical Research Laboratory (US); 1984 Jun. Report No.: NSMRL-1021. Available from: http://archive. rubicon-foundation.org/8416. [cited 2017 April 11].

2 Walker, R. A complete sbmarine escape and rescue organisation. SPUMS Journal. 1997;27:95-101.

3 Royal Australian Navy SUBSAFE guide. Canberra: Department of Defence; 1995. Classified.

4 Royal Australian Navy SUBSUNK Medical Guide. Canberra: Department of Defence; 2015. Available from: http://drnet. defence.gov.au/navy/FLD/FleetHealth/Pages/Policy\%20 and\%20Organisational\%20Links.aspx (restricted access). [cited 2017 Apr 11].

5 Royal Australian Navy. Numerical investigation into the compartment flood level during rush escape. Canberra (ACT): Department of Defence (AUS); 2011. Report No.: 1058246. Sponsored by the Department of Defence. (classified)

6 Royal Australian Navy diving manual. Canberra: Department of Defence; 2010. p.183.

7 DCIEM diving manual. North York (ON): Defense and Civil Institute of Environmental Medicine.; 1992. p. 60.

8 National Oceanic and Atmospheric Administration Diving Manual. Maryland: United States Department of Commerce; 2001. p. 17.7.

9 Gerth WA, Doolette DJ. VVal-18 and VVal-18M Thalmann algorithm air decompression tables and procedures. Panama City (FL): United States Navy Experimental Dive Unit; 2007 May. Report No.: 07-09. Available from: http://archive. rubicon-foundation.org/8349. [cited 2017 April 11].

10 United States Navy diving manual, revision 6: Washington: Department of Defense; 2008. p. 992.

11 United Kingdom Military diving manual, volume 2. Hants: Ministry of Defence; 1999. p. 556.

12 North Atlantic Treaty Organisation. The submarine search and rescue manual: Brussels: ATP 57; 2009. p. 645.
13 Gerth WA. Oxygen-accelerated decompression of submarine rescue and diving recompression system (SRDRS) operators and tenders. Panama City (FL): Navy Experimental Dive Unit; 2005 Apr. Report No.: NEDU TR 05-04. Available from: http:// archive.rubicon-foundation.org/3485.

14 Reid M, Doolette D, Fock A. Decompressing inside recompression chamber attendants during Australian submarine rescue operations. Diving Hyperb Med. 2017;47:168-72.

15 Stephenseon RN, MacKenzie I, Watt SJ, Ross JA. Measurement of oxygen concentration in delivery systems used for hyperbaric oxygen therapy. Undersea Hyperb Med. 1996;23:185-8.

16 Parker EC, Survanshi SS, Massell PB, Weathersby PK. Probabilistic models of the role of oxygen in human decompression sickness. J Appl Physiol. 1998;84:1096-102.

17 Gerth WA, Vann RD. Probabilistic gas and bubble dynamics models of decompression sickness occurrence in air and nitrogenoxygen diving. Undersea Hyperb Med. 1997;24:275-92.

18 Bardin H, Lambertsen CJ. A quantitative method for calculating pulmonary oxygen toxicity. Use of the unit pulmonary toxicity dose. Institute for Environmental Medicine Report. Philadelphia: University of Pennsylvania; 1970 Apr. [cited 2017 Aug 11]. Available from: http://archive.rubiconfoundation.org/10898.

19 Harabin AL, Homer LD, Weathersby PK, Flynn ET. An analysis of decrements in vital capacity as an index of pulmonary oxygen toxicity. J Appl Physiol. 1987;63:11301135.

20 Jamil SM, Spragg RG. Acute lung injury: acute respiratory distress syndrome. In: Papadakos PJ, Lachman B, editors. Mechanical ventilation clinical applications and pathophysiology. Philadelphia: Saunders; 2008. p. 28-39.

21 Hamilton RW, Kenyon DJ, Peterson RE. Repetitive excursion habitat diving procedures: repetitive vertical excursions, oxygen limits, and surfacing techniques. Rockville (MA): National Undersea Research Program; 1988 May Report No.: 88-1B. Available from: http://archive.rubicon-foundation. org/4866. [cited 2017 April 11].

22 Lauckner GR, Nishi RY, Eatlock BC. Evaluation of the DCIEM 1983 decompression model for compressed air diving (Series A-F). North York (ON): Defense and Civil Institute for Environmental Medicine; 1984 Oct. Report No.: 84-R-72. Available from: http://archive.rubicon-foundation.org/4283. [cited 2017 April 11].

23 Lauckner GR, Nishi RY, Eatlock BC. Evaluation of the DCIEM 1983 decompression model for compressed air diving (Series G-K). North York (ON): Defense and Civil Institute for Environmental Medicine; 1984 Nov. Report No.: 84-R-73. Available from: http://archive.rubicon-foundation.org/4284. [cited 2017 April 11].

24 Lauckner GR, Nishi RY, Eatlock BC. Evaluation of the DCIEM 1983 decompression model for compressed air diving (Series L-Q). North York (ON): Defense and Civil Institute for Environmental Medicine; 1985 Apr. Report No.: 85-R-18. Available from: http://archive.rubicon-foundation.org/4285. [cited 2017 April 11].

25 Gerth WA, Doolette, DJ. Schedules in the integrated air decompression table of U.S Navy diving manual, revision 6: Computation and estimated risks of decompression sickness. Panama City (FL): Navy Experimental Dive Unit; 2009 Jun. Report No.: NEDU TR 09-05. Available from: http://archive. rubicon-foundation.org/9898. [cited 2017 April 11]. 
26 Thalmann ED. Suitability of the United States Navy MK 15 (VVAL 18) decompression algorithm for air diving. Panama City (FL): United States Navy Experimental Dive Unit; 2003 Aug. Report No.: 03-12. Available from: http://archive. rubicon-foundation.org/4586. [cited 2017 April 11].

27 Manning E. Central nervous system oxygen toxicity and hyperbaric oxygen seizures. Aerosp Med Hum Perform. 2016;87:477-86.

\section{Acknowledgements}

Technical advice was provided by Mark Carey (James Fisher Defence), Kate Ponton (Defence Science and Technology Organisation), Brett Westcott (Submarine Escape and Rescue Manager), John Pennefather (Submarine Underwater Medicine Unit) and Wayne A Gerth (Navy Experimental Diving Unit). Lieutenant Commander Giselle Mouret (RANR, deceased) kindly provided French translation services.
Funding

The Royal Australian Navy provided funding for a USA visa for MPR.

Conflicts of interest: nil

Ethics statements

The Australian Defence Human Research Ethics Committee (ADHREC) confirmed in an email dated 23 October 2012 that this study was exempt from ethical review. The opinions expressed in this paper are those of the authors and do not necessarily reflect those of the Royal Australian Navy or US Department of the Navy.

Submitted: 30 August 2016; revised 21 January and 14 May 2017 Accepted: 22 June 2017

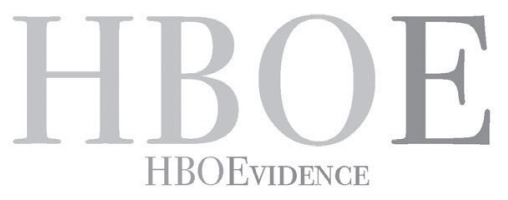

Available on the

App Store

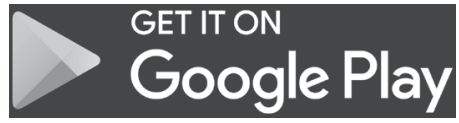

The database of randomised controlled trials in diving and hyperbaric medicine maintained by Michael Bennett and his colleagues at the Prince of Wales Hospital Diving and Hyperbaric Medicine Unit, Sydney is at:

<http://hboevidence.unsw.wikispaces.net/>

\author{
Assistance from interested physicians in preparing critical appraisals (CATs) is \\ welcomed, indeed needed, as there is a considerable backlog. \\ Guidance on completing a CAT is provided. \\ Contact Professor Michael Bennett: <m.bennett@unsw.edu.au>
}




\title{
Decompressing recompression chamber attendants during Australian submarine rescue operations
}

\author{
Michael P Reid ${ }^{1}$, Andrew Fock², David J Doolette ${ }^{3}$ \\ ${ }^{1}$ Submarine Underwater Medicine Unit, Royal Australian Navy, Sydney, Australia \\ ${ }^{2}$ Hyperbaric Unit, The Alfred Hospital, Melbourne, Australia \\ ${ }^{3}$ Navy Experimental Diving Unit, United States Navy, Panama City, USA
}

Corresponding author: Michael Reid, John Hunter Hospital, Department of Anaesthesia, Locked Bag 1, Hunter Region Mail Centre, NSW, 2310, Australia

mreid11@me.com

\section{Key words}

Decompression tables; Decompression sickness; Probability; Models; Oxygen; Military diving; Environmental medicine

\begin{abstract}
(Reid MP, Fock A, Doolette DJ. Decompressing recompression chamber attendants during Australian submarine rescue operations. Diving and Hyperbaric Medicine. 2017 September;47(3):168-172.)

Introduction: Inside chamber attendants rescuing survivors from a pressurised, distressed submarine may themselves accumulate a decompression obligation which may exceed the limits of Defense and Civil Institute of Environmental Medicine tables presently used by the Royal Australian Navy. This study assessed the probability of decompression sickness $\left(\mathrm{P}_{\mathrm{DCS}}\right)$ for medical attendants supervising survivors undergoing oxygen-accelerated saturation decompression according to the National Oceanic and Atmospheric Administration (NOAA) 17.11 table.

Methods: Estimated probability of decompression sickness $\left(\mathrm{P}_{\mathrm{DCS}}\right)$, the units pulmonary oxygen toxicity dose (UPTD) and the volume of oxygen required were calculated for attendants breathing air during the NOAA table compared with the introduction of various periods of oxygen breathing.

Results: The $\mathrm{P}_{\mathrm{DCS}}$ in medical attendants breathing air whilst supervising survivors receiving NOAA decompression is up to $4.5 \%$. For the longest predicted profile ( 830 minutes at $253 \mathrm{kPa}$ ) oxygen breathing at 30,60 and 90 minutes at $132 \mathrm{kPa}$ partial pressure of oxygen reduced the air-breathing-associated $\mathrm{P}_{\mathrm{DCS}}$ to less than $3.1 \%, 2.1 \%$ and $1.4 \%$ respectively.

Conclusions: The probability of at least one incident of DCS among attendants, with consequent strain on resources, is high if attendants breathe air throughout their exposure. The introduction of 90 minutes of oxygen breathing greatly reduces the probability of this interruption to rescue operations.
\end{abstract}

\section{Introduction}

The ambient pressure inside a distressed submarine (DISSUB) may be elevated above $101 \mathrm{kPa}$ due to compression of the remaining gas space by partial flooding or released high-pressure gas supplies. ${ }^{1}$ Locating the DISSUB and delivering the rescue system on to the site may take several days resulting in the crew of the DISSUB becoming saturated (inert gas tissue tension equilibration with the inspired inert gas pressures) at elevated pressure. As detailed in a companion paper, ${ }^{2}$ the submarine crew may be rescued by a Submersible Rescue Vehicle (SRV) which can mate to the DISSUB's escape hatch.

Survivors can transfer under pressure to a recompression chamber (RCC) at the surface where they undergo saturation decompression from the DISSUB internal pressure. ${ }^{2}$ Royal Australian Navy (RAN) DISSUB planning assumes all souls could be rescued from a DISSUB pressure of $253 \mathrm{kPa}$ (2.5 bar). Higher DISSUB pressures are possible, but RAN analysis of Collins Class submarines (classified) has assessed that likely conditions associated with such pressures are not survivable, and would require the crew to escape rather than await rescue. ${ }^{3-5}$

The current RAN rescue system uses the James Fisher Defence 'LR5' SRV which can rescue up to 14 seated survivors per sortie, a transfer under pressure (TUP) compartment, and two RCCs that can accommodate seven survivors and one medical attendant each (see accompanying paper for more details ${ }^{2}$ ). Separate medical personnel attend survivors inside the SRV, the TUP compartment, and inside each RCC. Decompression of the SRV and TUP medical attendants was the subject of the companion paper. ${ }^{2}$ The hyperbaric exposure for RCC medical attendants begins with pressurization of the RCC to DISSUB internal pressure ready for transfer under pressure of survivors. The RCC attendant hyperbaric exposure consists of 60 to 150 minutes (min) at the equivalent to DISSUB internal pressure, while survivors are transferred under pressure from the SRV to the RCCs, plus the time required for saturation decompression.

The National Oceanic and Atmospheric Administration (NOAA) 17.11 standard table is currently favoured by the 
RAN for oxygen-accelerated saturation decompression of survivors, owing to its relatively short total decompression time (TDT) of $680 \mathrm{~min}$ from a $253 \mathrm{kPa}$ air-saturation depth. ${ }^{6}$ The RCC medical attendants may, therefore, be exposed to hyperbaric pressure for a total of 740 to $830 \mathrm{~min}$. Unlike survivors, the RCC medical attendants' duties will prevent them from remaining at rest, and they therefore cannot breathe oxygen $\left(\mathrm{O}_{2}\right)$ throughout the decompression. These RCC medical attendants will themselves be exposed to the risk of decompression sickness (DCS).

Each cohort of fourteen survivors must be decompressed before the next SRV sortie is completed, and six or more sorties may be required to evacuate the DISSUB of rescue personnel and survivors, particularly if some survivors are immobilised secondary to their injuries. With the current RAN rescue plan, inside chamber attendants assisting survivors will be required to supervise two saturation decompressions separated by a 34-hour (h) surface interval.

As the NOAA tables were originally designed for decompressing uninjured scientific divers from underwater habitats, there are no accompanying instructions within the NOAA diving manual on how to decompress supervising attendants. ${ }^{6}$ At present, the only tables authorized for decompression of RAN medical attendants are the Defense and Civil Institute of Environmental Medicine (DCIEM) tables. ${ }^{7,8}$ These tables were designed for underwater diving operations and have a table limit of $280 \mathrm{~min}$ at 253 $\mathrm{kPa}$ (DCIEM 2 Table). ${ }^{8}$ In order to supervise survivors during $830 \mathrm{~min}$ in the $\mathrm{RCC}$, it would require a new attendant to be locked-in after each 280 -min period, and the previous attendant decompressed according to the DCIEM schedule. This is impractical for supervising the saturation decompression, as it would require three attendants to be rotated through each $\mathrm{RCC}$, or six attendants in total, to supervise survivors from each SRV sortie. These human resource constraints prompted an investigation into the DCS risk for RCC attendants supervising the NOAA 17.11 schedule and whether the introduction of $\mathrm{O}_{2}$ breathing periods could mitigate this risk and avoid the need to decompress with DCIEM tables.

\section{Methods}

The approach was to analyse hypothetical dive profiles (pressure/time/breathing gas histories) representing RCC attendant hyperbaric exposures whilst supervising saturation decompression from $253 \mathrm{kPa}$. Dive profiles were for either 60 or $150 \mathrm{~min}$ exposure at $253 \mathrm{kPa}$, followed by the 680 min of decompression stops required by the NOAA 17.11 table for a $253 \mathrm{kPa}$ saturation depth. Different dive profiles represented either air breathing throughout the exposure or incorporating periods of $\mathrm{O}_{2}$ breathing.

The methods of analysing the dive profiles are covered in detail in the companion paper in this issue and given here in summary. ${ }^{2}$ The instantaneous risk and the probability of DCS $\left(\mathrm{P}_{\mathrm{DCS}}\right)$ for each dive profile was calculated using the Navy Medical Research Institute 98 (NMRI-98, Model 2) and Bubble Volume Model $3[\mathrm{BVM}(3)]$ probabilistic models. ${ }^{9,10}$ In the NMRI-98 model, instantaneous risk of DCS is a function of the gas supersaturation in three modelled tissue compartments. ${ }^{9}$ In the BVM(3) model, instantaneous risk is a function of the bubble volume in three modelled tissue compartments. ${ }^{10}$ The $\mathrm{P}_{\mathrm{DCS}}$ is a function of the time-integral of these instantaneous risks. ${ }^{9,10}$ In this paper the instantaneous risk was used to guide scheduling of $\mathrm{O}_{2}$ breathing periods.

The oxygen consumption and units pulmonary toxicity dose (UPTD) were calculated for each of these dive profiles using an equation derived from the Harabin et al. method. ${ }^{11} \mathrm{O}_{2}$ usage per attendant was based on a conservative respiratory minute ventilation rate of 15 litres $\cdot \mathrm{min}^{-1}$, adjusted for Boyles Law and body temperature and pressure, saturated (BTPS). This is a deliberate over-estimation, based on a $10 \mathrm{ml} \cdot \mathrm{kg}^{-1}$ tidal volume, resting adult respiratory rate of 15 breaths. $\mathrm{min}^{-1},{ }^{12}$ and a body weight of $100 \mathrm{~kg}$.

\section{Results}

Figure 1 shows the dive profile for $150 \mathrm{~min}$ at $253 \mathrm{kPa}$ followed by the NOAA 17.11 decompression stops. The upper panel shows the time course of the BVM(3)-estimated instantaneous risk for this profile if the attendant breathes air throughout. This risk occurs after decompression from 132 to $117 \mathrm{kPa}$ (10 to 5 feet' seawater, fsw) and after decompression to the surface. The lower panel illustrates the effect of introducing attendant $\mathrm{O}_{2}$ breathing for the entirety of the $132 \mathrm{kPa}$ decompression stop (90 min). This $\mathrm{O}_{2}$ breathing eliminates the $\mathrm{BVM}(3)$ estimated instantaneous risk at $117 \mathrm{kPa}$ and greatly reduces the magnitude and duration of the risk at the surface. Similarly, NMRI-98 estimated instantaneous risk (not shown) occurred principally after decompression from 132 to $117 \mathrm{kPa}$ and after decompression to the surface. Once again DCS risk was reduced by $\mathrm{O}_{2}$ breathing at $132 \mathrm{kPa}$. These results prompted evaluation of the $\mathrm{P}_{\mathrm{DCS}}$ when different periods of oxygen breathing were introduced at $132 \mathrm{kPa}$.

Figure 2 shows the estimated $\mathrm{P}_{\mathrm{DCS}}(\%)$ for RCC medical attendants supervising survivors during the NOAA 17.11 Table following 60 or $150 \mathrm{~min}$ at $253 \mathrm{kPa}$ with total hyperbaric times of 740 and 830 min respectively. It compares air decompression with varying periods of $\mathrm{O}_{2}$ breathing ( $3 \mathrm{~min}, 30 \mathrm{~min}, 60 \mathrm{~min}$ and $90 \mathrm{~min}$ ) at $132 \mathrm{kPa}$ ending just prior to ascent to $117 \mathrm{kPa}$. $\mathrm{O}_{2}$ breathing at this depth reduced estimated $\mathrm{P}_{\text {DCS }}$ from between $3.8 \%$ to $4.4 \%$ using air to $1.2 \%$ to $1.3 \%$ with $90-\mathrm{min}_{2}$ breathing for an 830-min dive.

We did not directly estimate $\mathrm{P}_{\mathrm{DCS}}$ for repetitive $\mathrm{RCC}$ medical attendant exposures under the assumption that the 34-h 
Figure 1

$\mathrm{BVM}(3)$ estimated instantaneous risk of DCS for RCC attendants supervising the NOAA 17.11 table whilst breathing air. Total exposure is 830 min commencing with $150 \mathrm{~min}$ at $253 \mathrm{kPa}$; the secondary y-axis is instantaneous DCS risk in arbitrary units representing bubble volumes in three modelled compartments. The upper panel represents air breathing throughout, the lower panel is air breathing with a single $90 \mathrm{~min}$ of $\mathrm{O}_{2}$ breathing at $132 \mathrm{kPa}$, indicated by the horizontal bar. The recompression just prior to surfacing reflects the origin of these schedules for decompression from sea-floor habitats for which recompression is required to allow divers to exit the habitat into the water
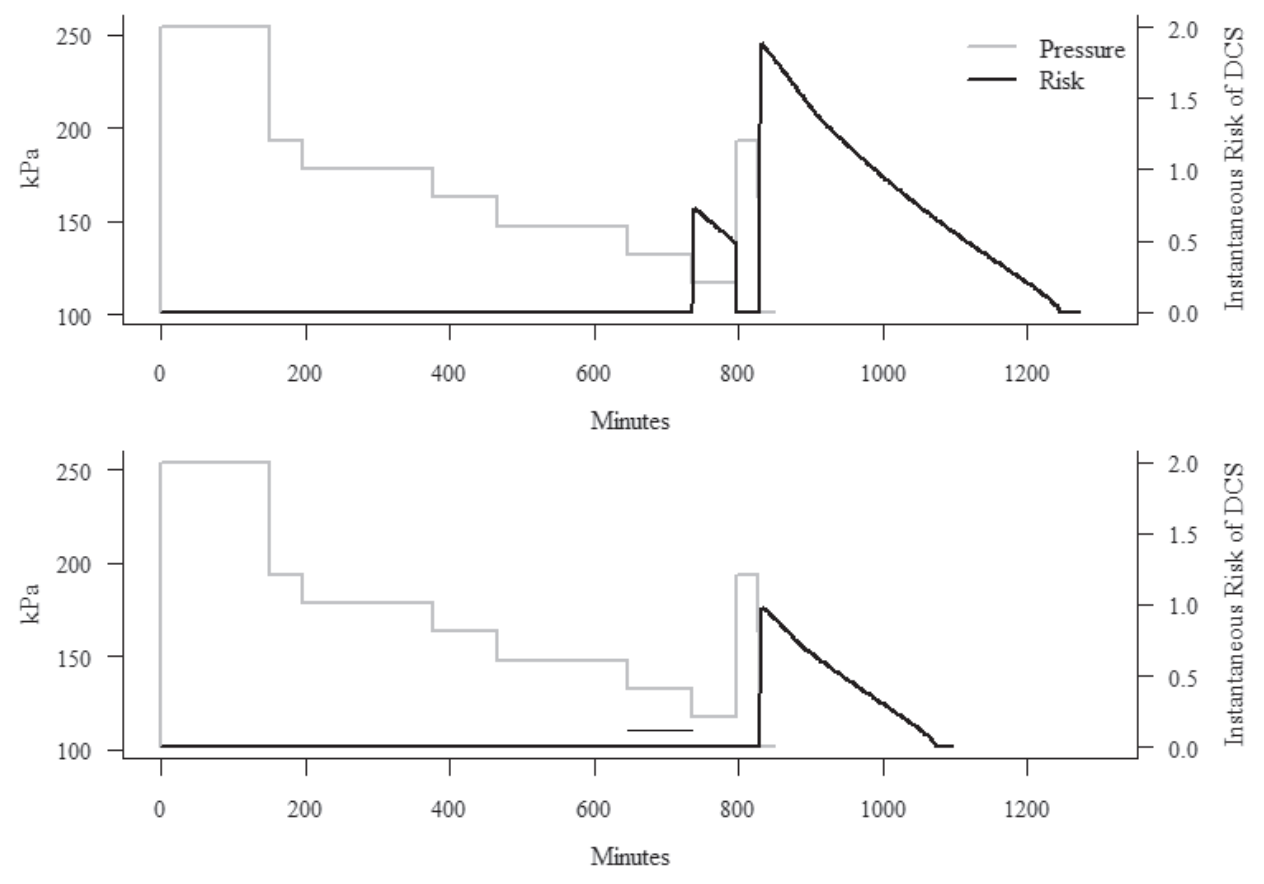

Figure 2

$\mathrm{P}_{\mathrm{DCS}}(\%)$ for medical attendants for single dives with bottom times of 740 and $830 \mathrm{~min}$ at $253 \mathrm{kPa}$ estimated using the NMRI98 and BVM3 model for the NOAA standard table; at each bottom time the cluster of bars gives the $\mathrm{P}_{\mathrm{DCS}}$ for decompression

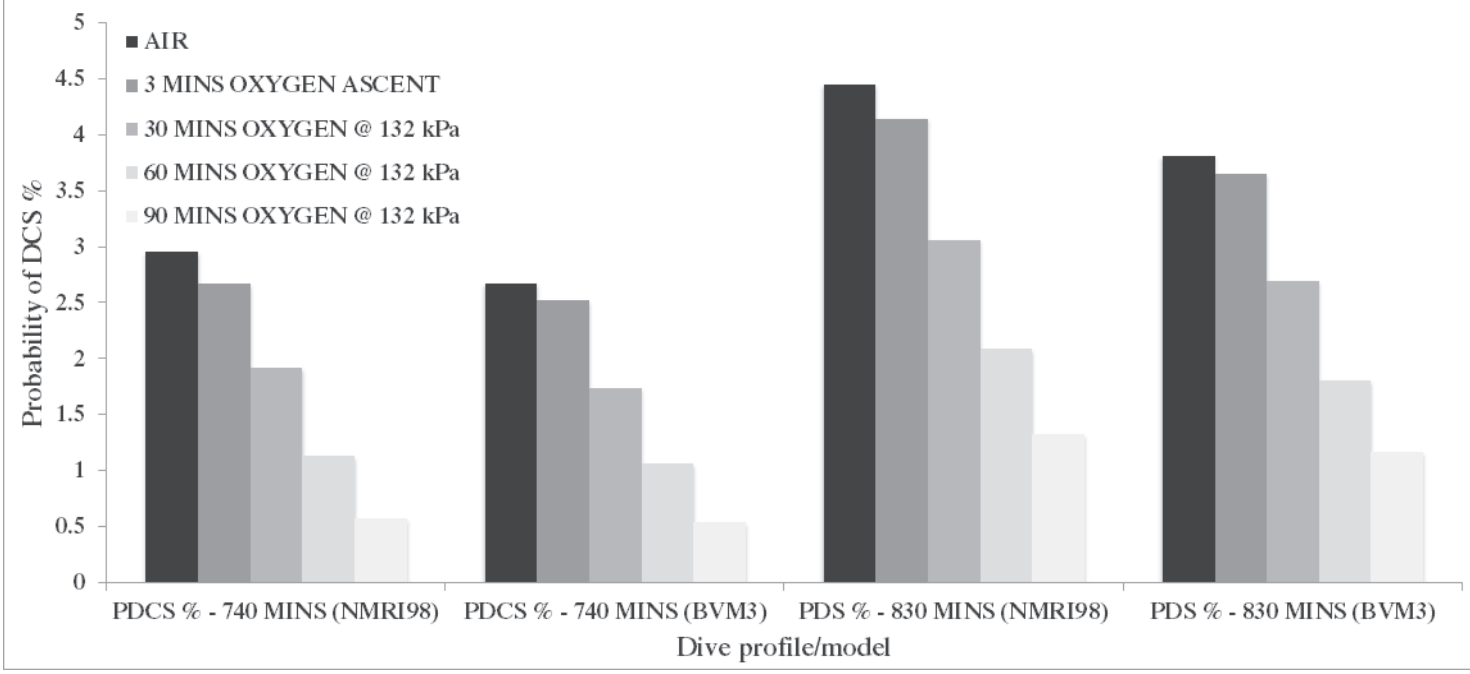

surface interval was sufficient that attendants would be 'clean' for each dive. The probability of at least one incident of DCS in a series of identical dives can be determined using binomial theorem, and is one minus the probability of no DCS in all dives. Using the BVM(3) estimate of 3.8\% $\mathrm{P}_{\mathrm{DCS}}$ (830-min exposure with air decompression), RCC attendants performing two such exposures will have a probability of a DCS case of:

$$
1-(1-0.038)^{2}=7.5 \%
$$

Twelve RCC attendant exposures will be required to rescue 65 survivors and the probability of at least one DCS case among all RCC attendants performing such exposures is:

$$
1-(1-0.038)^{12}=37.2 \%
$$


Table 1

Cumulative unit pulmonary toxicity dose (CUPTD) for NOAA attendants after 740- and 830-min dives commencing at $253 \mathrm{kPa}$ and total oxygen $\left(\mathrm{O}_{2}\right)$ usage: breathing air and with $3 \mathrm{~min}, 30 \mathrm{~min}, 60 \mathrm{~min}$ and $90 \mathrm{~min}$ of $\mathrm{O}_{2}$, all at $132 \mathrm{kPa}$ just prior to ascent to $117 \mathrm{kPa}$ pressure

Oxygen breathing period
None
3 min final ascent
$30 \mathrm{~min} @ 132 \mathrm{kPa}$
$60 \mathrm{~min} @ 132 \mathrm{kPa}$
$90 \mathrm{~min} @ 132 \mathrm{kPa}$

CUPTD 740 min
2
6
35
68
101

CUPTD 830 min
4
8
37
71
104

Oxygen use (L)

0
86
862
1,723
2,585

The use of 90 min of oxygen breathing reduced the $\mathrm{P}_{\mathrm{DCS}}$ of the 830-min RCC attendant exposure to $1.2 \%$, and this reduces the probability of at least one case of DCS in two exposures to $2.4 \%$ and in twelve exposures to $13.5 \%$. These figures illustrate the advantages of adopting conservative decompression for rescue personnel.

Table 1 shows the UPTD for RCC medical attendants after 740 and 830-min dives commencing at $253 \mathrm{kPa}$ whilst breathing air, and for the four different periods of $\mathrm{O}_{2}$ breathing. Table 1 also shows the estimated $\mathrm{O}_{2}$ usage for a single attendant for these $\mathrm{O}_{2}$ periods. None of these UPTD exceeded Repetitive Excursion (REPEX) recommendations. ${ }^{13}$

\section{Discussion}

Probabilistic decompression model estimates of the $\mathrm{P}_{\mathrm{DCS}}$ for attendants supervising United States Navy (USN) treatment tables have been reported. ${ }^{14}$ The estimated $\mathrm{P}_{\mathrm{DCS}}$ for Treatment Table 6 ranges from $6.2 \%$ to $11.2 \%$, depending on the number of extensions for attendants breathing air throughout. ${ }^{15,16}$ The introduction of periods of $\mathrm{O}_{2}$ breathing for the attendant decreases the estimated $\mathrm{P}_{\mathrm{DCS}}$ to near $0 \% .{ }^{15}$ Such $\mathrm{O}_{2}$ decompression of attendants has been adopted by many organisations including the RAN, ${ }^{7}$ and the same probabilistic modelling was used to investigate $\mathrm{P}_{\mathrm{DCS}}$ in attendants supervising the NOAA 17.11 table during submarine rescue.

The RAN does not have a policy on acceptable $\mathrm{P}_{\text {DCS }}$ for diving or DISSUB rescue operations. However, the DCIEM tables, which are approved for RAN use, had a DCS incidence of $3.2 \%$ to $3.5 \%$ during development and validation. ${ }^{16-19}$ Most USN air and nitrox decompression procedures have an upper limit of $5 \% \mathrm{P}_{\mathrm{DCS}}$ for normal exposure diving. ${ }^{20,21}$ The highest estimated $\mathrm{P}_{\mathrm{DCS}}$ for RCC medical attendants breathing air throughout decompression was $4.4 \%$, toward the upper end of the normal exposure air diving range. RCC attendants need to perform at least two dives, separated by a 34-h surface interval, and collectively, twelve RCC attendant exposures will be required to rescue 65 survivors. The probability of a single DCS incident in the course of a series of dives is greater than the $\mathrm{P}_{\mathrm{DCS}}$ of a single dive and any DCS in rescue personnel will result in a serious strain on resources.
It is neither necessary nor desirable for RCC medical attendants to breathe $\mathrm{O}_{2}$ throughout the decompression, as do the survivors, as attendants have a lower decompression obligation and their duties inside the chamber put them at greater risk of central nervous system (CNS) oxygen toxicity than the survivors. RCC medical attendant $\mathrm{O}_{2}$ breathing should be later in the decompression cycle to minimise the re-uptake of nitrogen during any subsequent hyperbaric air breathing and scheduled at the shallowest decompression stop so as to minimise $\mathrm{O}_{2}$ toxicity and $\mathrm{O}_{2}$ usage. Scheduling $\mathrm{O}_{2}$ breathing to occur at $132 \mathrm{kPa}(10 \mathrm{fsw})$ fulfils these objectives.

The highest UPTD dose of 104 units does not exceed REPEX recommendations. ${ }^{13}$ The risk of $\mathrm{CNS} \mathrm{O}_{2}$ toxicity is considered low as the deepest decompression stop whilst breathing oxygen is $132 \mathrm{kPa}$ (Table 1). Within a dry environment, the risk of $\mathrm{O}_{2}$ toxicity seizures ranges from $1: 1,000$ to $1: 50,000 .^{22}$

The introduction of 90 min of $\mathrm{O}_{2}$ breathing for $\mathrm{RCC}$ medical attendants requires an additional 2,585 litres of $\mathrm{O}_{2}$ per person (Table 1), of which there are sufficient $\mathrm{O}_{2}$ supplies held onboard existing vessels. Assessing the effect of breathing $\mathrm{O}_{2}$ on ascent ( $3 \mathrm{~min}$ ) and at 30 and $60 \mathrm{~min}$ at $132 \mathrm{kPa}$ provides options in case of critical $\mathrm{O}_{2}$ supply constraints.

\section{Conclusions}

RCC medical attendants supervising saturation decompression of DISSUB survivors are themselves at risk of DCS, and if breathing air for the entirety of their hyperbaric exposure, this risk is considered high. RCC medical attendants are critical to the success of the rescue operation and any DCS occurring within this cohort will place further strain on chamber space availability and human and oxygen supply resources, Introducing $90 \mathrm{~min}$ of $\mathrm{O}_{2}$ breathing at $132 \mathrm{kPa}$ greatly reduces the probability of this disruption to the rescue operation.

\section{References}

1 Eckenhoff RG. Pressurized submarine rescue. Groton (CT): Navy Submarine Medical Research Laboratory (US), 1984 Jun. Report No.: NSMRL-1021. Available from: http://archive. rubicon-foundation.org/8416. [cited 2014 Jan 07]. 
2 Reid M, Doolette D, Fock A. Decompressing rescue personnel during Australian submarine rescue operations. Diving Hyperb Med. 2017;47:159-167.

3 Royal Australian Navy SUBSAFE Guide, 1995 Oct. Report No.: N93-24191. Canberra: Department of Defence; 1995. Sponsored by the Department of Defence (restricted access). [cited 2017 Apr 21].

4 Royal Australian Navy SUBSUNK medical guide. Canberra (ACT): Royal Australian Navy; 2015 Dec. Available from: http://drnet.defence.gov.au/navy/FLD/FleetHealth/Pages/ Policy\%20and\%20Organisational\%20Links.aspx (restricted access). [cited 2017 Apr 11].

5 Royal Australian Navy numerical investigation into the compartment flood level during rush escape. Canberra (ACT): Department of Defence (AUS); 2011. Report No. 1058246. Sponsored by the Department of Defence. (classified)

6 National Oceanic and Atmospheric Administration. Diving manual. Maryland: United States Department of Commerce; 2001. p. 17.7.

7 Royal Australian Navy. Diving manual. Canberra: Department of Defence; 2010. p.183.

8 Defense and Civil Institute of Environmental Medicine. Diving Manual. North York (ON): Defense and Civil Institute of Environmental Medicine; 1992. p. 60.

9 Parker EC, Survanshi SS, Massell PB, Weathersby PK. Probabilistic models of the role of oxygen in human decompression sickness. J Appl Physiol. 1998;84:1096-102.

10 Gerth WA, Vann RD. Probabilistic gas and bubble dynamics models of decompression sickness occurrence in air and nitrogen-oxygen diving. Undersea Hyperb Med. 1997; 24: 275-92.

11 Harabin AL, Homer LD, Weathersby PK, Flynn ET. An analysis of decrements in vital capacity as an index of pulmonary oxygen toxicity. J Appl Physiol. 1987;63:1130-5.

12 Jamil SM, Spragg RG. Acute lung injury: acute respiratory distress syndrome. In: Papadakos PJ, Lachman B, editors. Mechanical ventilation clinical applications and pathophysiology. Philadelphia: Saunders; 2008. p. 28-39.

13 Hamilton RW, Kenyon DJ, Peterson RE. Repetitive excursion habitat diving procedures: repetitive vertical excursions, oxygen limits, and surfacing techniques. Rockville (MA): National Undersea Research Program; 1988 May. Report No.: 88-1B. Available from: http://archive.rubicon-foundation. org/4866. [cited 2014 Jan 07].

14 Thalmann, ED. Principles of U.S. Navy recompression treatments for decompression sickness. In: Moon RE, Sheffield PJ, editors. Treatment of decompression illness. 45th UHMS Workshop, Kensington, MD: Undersea and Hyperbaric Medical Society, 1996; p. 75-95. Available from: http:// archive.rubicon-foundation.org/7999. [cited 2017 April 21].

15 Sheffield PJ, Pirone CJ. Decompression sickness in inside attendants. In: Workman, WT, editor. Hyperbaric facility safety; a practical guide. North York, ON: Best Publishing Company; 2000. p. 643.

16 Nishi RY, Tikuisis P. Current trends in decompression development: statistics and data analysis. North York (ON): Defense and Civil Institute of Environmental Medicine; 1996. Report No.: DCIEM 96-R-65. Available from: http://archive. rubicon-foundation.org/3870. [cited 2014 Jan 07].

17 Lauckner GR, Nishi RY, Eatlock BC. Evaluation of the DCIEM 1983 decompression model for compressed air diving (Series A-F). North York (ON): Defense and Civil Institute for Environmental Medicine; 1984 Oct. Report No.: 84-R-72. Available from: http://archive.rubicon-foundation.org/4283. [cited 2014 Jan 07].
18 Lauckner GR, Nishi RY, Eatlock BC. Evaluation of the DCIEM 1983 decompression model for compressed air diving (Series G-K). North York (ON): Defense and Civil Institute for Environmental Medicine; 1984 Nov. Report No.: 84-R-73. Available from: http://archive.rubicon-foundation.org/4284. [cited 2014 Jan 07].

19 Lauckner GR, Nishi RY, Eatlock BC. Evaluation of the DCIEM 1983 decompression model for compressed air diving (Series $L-Q)$. North York $(\mathrm{ON})$ : Defense and Civil Institute for Environmental Medicine; 1985 Apr. Report No.: 85-R-18. Available from: http://archive.rubicon-foundation.org/4285. [cited 2014 Jan 07].

20 Gerth WA, Doolette, DJ. Schedules in the integrated air decompression table of US Navy Diving Manual, Revision 6: Computation and estimated risks of decompression sickness. Panama City (FL): Navy Experimental Dive Unit; 2009 Jun. Report No.: NEDU TR 09-05. Available from: http://archive. rubicon-foundation.org/9898. [cited 2014 Jan 07].

21 Thalmann ED. Suitability of the United States Navy MK 15 (VVAL 18) decompression algorithm for air diving. Panama City (FL): Navy Experimental Dive Unit; 2003 Aug. Report No.: NEDU TR 03-12. Available from: http://archive.rubiconfoundation.org/4586. [cited 2014 Jan 07].

22 Manning E. Central nervous system oxygen toxicity and hyperbaric oxygen seizures. Aerospace Med Hum Perform. 2016;87:477-86.

\section{Acknowledgements}

Technical advice was provided by Mark Carey (James Fisher Defence), Kate Ponton (Defence Science and Technology Organisation), Brett Westcott (Submarine Escape and Rescue Manager), John Pennefather (Submarine Underwater Medicine Unit) and Wayne A Gerth (Navy Experimental Diving Unit). The late Lieutenant Commander Giselle Mouret (RANR) kindly provided French translation services.

\section{Funding Source}

The Royal Australian Navy provided funding for a US visa for MPR.

\section{Conflict of interest: nil}

\section{Ethics statements}

The Australian Defence Human Research Ethics Committee (ADHREC) confirmed in an email dated 23 October 2012 that this study was exempt from ethical review. The opinions expressed in this paper are those of the authors and do not necessarily reflect those of the Royal Australian Navy or US Department of the Navy.

Submitted: 30 August 2016; revised 05 January and 26 June 2017 Accepted: 29 June 2017 


\title{
Turkish recreational divers: a comparative study of their demographics, diving habits, health and attitudes towards safety
}

\author{
Bengusu Mirasoglu, Samil Aktas \\ Istanbul Faculty of Medicine, Underwater and Hyperbaric Medicine Department
}

Corresponding Author: Bengusu Mirasoglu, Istanbul Tip Fakultesi, Sualti Hekimligi ve Hiperbarik Tip Anabilim Dali, Capa/Fatih, 34093 Istanbul, Turkey

bengusu.mirasoglu@istanbul.edu.tr

Key words

Scuba diving; Sex; Medical conditions; Decompression sickness; Menstruation; Pregnancy; Survey

\begin{abstract}
(Mirasoglu B, Aktas S. Turkish recreational divers: a comparative study of their demographics, diving habits, health and attitudes towards safety. Diving and Hyperbaric Medicine. 2017 September;47(3):173-179.)

Introduction: In Turkey, scuba diving has become more popular and accessible in the past decade and there has been a commensurate rise in the number of certified divers. This new generation of recreational divers has not been described in detail previously. The aim of this study was to profile this group, while investigating any gender differences and making comparisons with the global diving community.

Methods: Turkish dive club members and diving forum/blog readers were invited to complete an online questionnaire investigating their demography, medical issues and diving history and habits.

Results: The questionnaire was completed by 303 female and 363 male divers. Significant differences were found between the sexes in terms of demographics, diving experience and attitudes toward safety. Previous or ongoing medical conditions were reported by 100 female divers and 141 males. Only $29 \%$ of females and $22 \%$ of males had been examined by a physician trained to conduct assessments of fitness to dive. Female divers did not report problems while diving during menstruation or while taking oral contraceptives. There was no significant difference in the occurrence of decompression sickness (DCS) and DCS-like symptoms between the sexes.

Conclusion: This is the largest study to date conducted on recreational divers in Turkey and so carries some value. It profiles their physical and behavioral attributes as well as differences in diving practices between the sexes in Turkey. Our findings should have implications for medical screening and dive training standards.
\end{abstract}

\section{Introduction}

Scuba diving is a popular recreational pursuit. In the USA, it is estimated that 3,145,000 divers made at least 9,396,000 dives in 2015 alone. ${ }^{1}$ According to PADI statistics, approximately 900,000 divers are certified each year. ${ }^{2}$ In countries like the UK and Australia, where diving has a longstanding background, many studies on diver demographics, behaviour and accident analyses have been published. ${ }^{3,4}$

In Turkey, however, scuba diving has become popular in only the past decade. The number of beginner divers is currently more than 5,000 per year, whereas before 2005, it was in the hundreds only. ${ }^{5}$ According to the Turkish Underwater Sports Federation's (TSSF) 2014 records, the number of certified divers has increased from 66,283 to 101,377 since 2010 . $^{6}$ This 'new' group of recreational divers has not been studied before so, given the increasing number of participants, a report seemed timely. Females account for a large part of this increase in the diver population. Currently, $21 \%$ of certified divers in Turkey are females, whereas they made up only $8 \%$ in 2000.5 Worldwide, females are reported to make up $28 \%$ of all recreational divers, so the proportional increase of female divers in Turkey is starting to come into line with the global trend. ${ }^{7}$ This raises questions about sex differences and susceptibility to diving-related diseases, but studies on the topic are limited. ${ }^{8,9}$ Also, any differences in diving behaviour and safety between the sexes are of interest. We aimed to profile Turkish divers, particularly this younger group, their demographics, diving habits, health status, attitudes toward diving safety and any difference between the sexes, while comparing them with other 'experienced' diving communities and their practices.

\section{Methods}

The study was approved by the Institutional Review Board of Istanbul University, Istanbul Faculty of Medicine, Turkey. From the 01 May until 16 June 2010, a weblink for a Turkish language online questionnaire (80 questions), which took about 20 minutes to complete, was sent to dive club members and posted on internet blogs and diving forums in Turkey. Information regarding the aim of the questionnaire and use of the results were provided with the link. The questionnaire was anonymous but nicknames were requested to eliminate duplicates. It was possible to leave the questionnaire at any point and unless participants saved it at the end, a record was not generated. 


\section{Table 1}

Body mass index (BMI, $\mathrm{kg} \cdot \mathrm{m}^{-2}$ ) of 303 female and 363 male Turkish recreational divers responding to the survey; number and percentage shown

\begin{tabular}{lrlrll} 
& \multicolumn{2}{c}{ Female divers } & \multicolumn{2}{c}{ Male divers } & $P$-value \\
BMI $<25$ & 264 & $(87)$ & 139 & $(38)$ & $<0.0001$ \\
BMI 25-25.9 & 31 & $(10)$ & 181 & $(50)$ & $<0.0001$ \\
BMI 30-34.9 & 4 & $(1.3)$ & 33 & $(9)$ & $<0.0001$ \\
BMI $>35$ & 4 & $(1.3)$ & 10 & $(3)$ & 0.14
\end{tabular}

The first part of the survey investigated diver demography, asking questions on anthropometric characteristics, occupation, education, family and medical background. All of these questions allowed the responders to reply descriptively, apart from two on alcohol and tobacco use that required yes/no responses. The second part aimed to collect data on diving history and diving habits, including years diving, number of dives, diving experience and training. They were also asked about diving beyond the safe recreational limits advised by their tables/dive computers. The third part of the survey addressed current health status including chronic diseases, medications, and decompression sickness (DCS) and other diving-related diseases. A DCS episode diagnosed by a physician and treated in a recompression chamber was defined as confirmed DCS. Numbness, extremity weakness, tingling, paresthesia and pain after a dive were accepted as DCS-like symptoms. Most of the questions in this section required yes/no answers, although some invited descriptive answers. An additional section to be completed by diving instructors included their instructing history, how they trained and differences in their approach to female and male divers. After one and a half months of the questionnaire being live, the data were collected and evaluated. The records were reviewed for duplicates and incomplete answers. Replies that had more than five answers missing were excluded.

\section{ANALYSIS}

All demographic data were expressed as mean ( \pm standard deviation) or percentages, as appropriate; categorized data were given as percentages. Statistical analyses were performed with Medcalc ${ }^{\circledR}$ for Windows (version 11.2.1.0). Simple z-test and ' $N$ - 1 ' chi-squared tests were used to compare proportions. Student's $t$-test was used to compare paired values. Significance was accepted at $P<0.05$.

\section{Results}

Upon initial examination, 317 female divers and 381 male divers supplied responses. Records were scrutinized for completion and duplication, with 27 failing to fulfill completion criteria and five being duplicates. A total of 666 records, 303 female and 363 male, were analyzed. The mean age of females was $32 \pm 8$ years (range: 19-59) and for males was $36 \pm 10$ (range: 15-67). Males were significantly older than females $(P<0.001)$. Mean body mass index (BMI,
Figure 1

Diving certification level of 303 female and 363 male Turkish recreational divers responding to the survey (percentages shown)

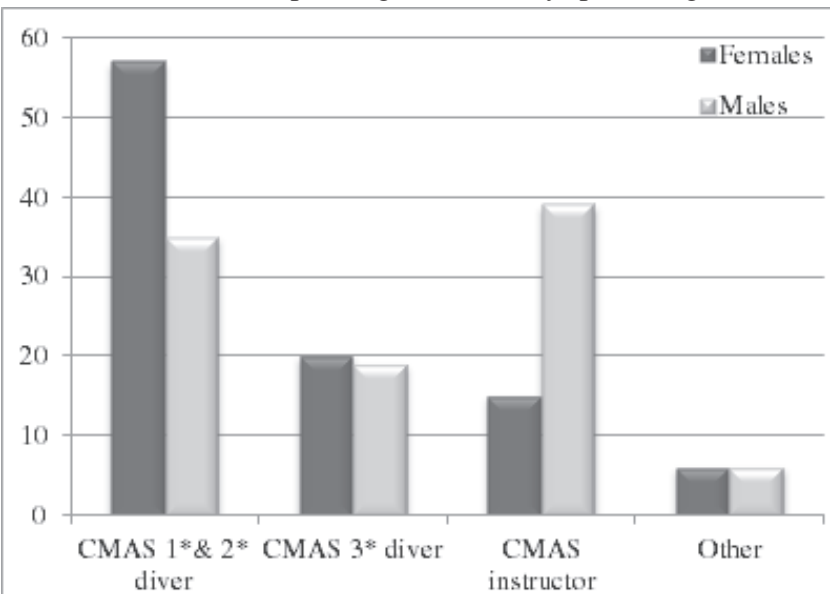

$\mathrm{kg} \cdot \mathrm{m}^{-2}$ ) was 22 for female and 26 for males. Eight female and forty-three male divers had a BMI $\geq 30$, categorizing them as obese. The distribution of BMI is given in Table 1 .

\section{DIVING HISTORY}

All divers replied to the question about diving certification (Figure 1). Details about diving history are given in Table $2 ; 17 \%$ of females and $9 \%$ of males had 20 or fewer dives. The total number of dives reported by females was 65,311 and 239,772 for males. $(P<0.0001)$. Males completed a significantly higher mean number of dives than females per year $(61 \pm 75$ vs $35 \pm 54, P<0.0001)$.

Diving behaviour is described in Table 3. Sixty-seven per cent of females $(n=204)$ and $81 \%$ of males $(n=294)$ declared they regularly made a safety stop, but only $57 \%$ of the females $(n=173)$ and $63 \%$ of males $(n=203)$ defined it as the 'industry standard' safety stop of 3-5 min at 3-6 metres' sea water (msw). Forty per cent of females $(n=121)$ and $62 \%$ of males $(n=225)$ reported using a dive computer to plan their dives.

Forty-two per cent of the female divers $(n=127)$ and $59 \%$ of males $(n=213, P<0.001)$ reported that they performed dives that required decompression stops. However, almost as many females did not reply to the question. Sixty-six per cent of female $(n=201)$ and $78 \%(n=284)$ of male divers did not adhere to the recreational diving depth limit, $30 \mathrm{msw}$ in Turkey $(P=0.0006) .{ }^{10}$ Moreover, another $11 \%(n=32)$ of female divers reported diving deeper than $55 \mathrm{msw}$, which is the limit for professional divers (with air) in Turkey. ${ }^{11}$

\section{HEALTH ISSUES}

Similar numbers of female and male divers were smokers (35\% and 34\% respectively). Ninety per cent of female and $84 \%$ of male divers reported that they consumed alcohol occasionally. Almost half of the females $(n=148)$ did or 
Table 2

Diving history of 303 female and 363 male Turkish divers responding to the survey; $n=$ number of respondents answering the question

\begin{tabular}{lccllccll} 
& \multicolumn{3}{c}{ Female divers } & \multicolumn{3}{c}{ Male divers } \\
& $n$ & Mean & $(\mathrm{SD})$ & Range & $n$ & Mean (SD) & Range \\
Years diving & 290 & 5.9 & $(4.4)$ & $2-25$ & 351 & 10 & $(8.1)$ & $3-48$ \\
Total dives & 271 & 241 & $(566)$ & $4-6000$ & 318 & 754 & $(1306)$ & $3-9000$ \\
Maximum depth (m) & 290 & 39.9 & $(14.3)$ & $8-89$ & 354 & 48.6 & $(17.7)$ & $8-137$
\end{tabular}

Table 3

Diving behaviour of 303 female and 363 male Turkish recreational divers responding to the survey (number of respondents and percentage shown)

\section{Female divers Male divers}

$\begin{array}{lrlrrr}\text { Safety stop } & n & (\%) & n & (\%) & P \text {-value } \\ \text { Always } & 204 & (67) & 294 & (81) & <0.0001 \\ \text { No response } & 74 & (24) & 45 & (12) & \\ \text { Decompression diving } & & & & \\ \text { Yes } & 127 & (42) & 213 & (59) & <0.0001 \\ \text { No } & 57 & (19) & 56 & (15) & \\ \text { No response } & 119 & (39) & 94 & (26) & \\ \text { Diving deeper than } \mathbf{3 0} \mathbf{~ m} & & & \\ \text { Yes } & 201 & (66) & 284 & (78) & 0.0006 \\ \text { No } & 35 & (12) & 31 & (9) & \\ \text { No response } & 67 & (22) & 48 & (13) & \\ \text { Diving deeper than } \mathbf{4 2} \mathbf{~ m} & & & \\ \text { Yes } & 116 & (38) & 248 & (68) & <0.0001 \\ \text { No } & 174 & (57) & 106 & (29) & \\ \text { No response } & 13 & (4) & 9 & (3) & \\ \text { Diving deeper than } \mathbf{5 5} \mathbf{~ m} & & & \\ \text { Yes } & 32 & (11) & 120 & (33) & <0.0001 \\ \text { No } & 258 & (85) & 234 & (64) & \\ \text { No response } & 13 & (4) & 9 & (3) & \end{array}$

would do so within 12 hours of diving, compared to $61 \%$ $(n=220)$ of the male respondents $(P=0.002)$.

Thirty-three per cent of females $(n=100)$ and $39 \%$ of males $(n=141)$ reported having an ongoing medical condition or having experienced one. For both sexes, the three most common medical conditions were ear, nose and throat problems, previous trauma and spinal disc herniation. Of the females, one reported having hypertension (HT) and hypercholesterolemia, one reported diabetes mellitus (DM) and HT, two reported cardiac problems (arrhythmia and rheumatic valve insufficiency) and one had hypoglycaemia. One female reported manic depression and had been evaluated by a general practitioner for diving. Of the male respondents, HT, DM or hypercholesterolemia were reported by five males, three of whom had two of these conditions at the same time. Three reported cardiac problems; previous surgery for aortic coarctation, arrhythmia and mitral valve prolapse. One male had a history of tuberculosis but had not been assessed for diving by a physician.

\section{Table 4}

Health issues and diving related accidents in 303 female and 363 male Turkish recreational divers responding to the survey (number of respondents and percentage shown)

\begin{tabular}{lrcrc} 
& Female divers & \multicolumn{2}{c}{ Male divers } \\
& $n$ & $(\%)$ & $n$ & $(\%)$ \\
Smoker & 105 & $(35)$ & 122 & $(34)$ \\
Alcohol & & & & \\
$\quad$ Occasional drinker & 274 & $(90)$ & 306 & $(84)$ \\
$\quad$ Never & 27 & $(9)$ & 43 & $(12)$ \\
Alcohol pre-dive & 148 & $(49)$ & 220 & $(61)$ \\
Exercise regularly & 45 & $(15)$ & 47 & $(13)$ \\
Medical assessment & & & & \\
Only before training & 168 & $(55)$ & 193 & $(53)$ \\
$\quad$ Regularly & 60 & $(20)$ & 111 & $(31)$ \\
Never & 63 & $(21)$ & 50 & $(14)$ \\
$\quad$ No response & 12 & $(4)$ & 9 & $(2)$ \\
Assessment by & & & & \\
$\quad$ Diving physician & 89 & $(29)$ & 143 & $(39)$ \\
Other (GP or specialist) & 133 & $(44)$ & 155 & $(43)$ \\
$\quad$ No response & 81 & $(27)$ & 65 & $(18)$ \\
DCS & & & & \\
Signs of DCS & 3 & $(1)$ & 11 & $(3)$ \\
Treated DCS & 1 & $(0.3)$ & 4 & $(1)$ \\
Other & & & & \\
$\quad$ Non-DCS trauma & 15 & $(5)$ & 27 & $(7)$ \\
Barotrauma & 8 & $(3)$ & 13 & $(4)$ \\
Hip/shoulder pain & 11 & $(4)$ & 31 & $(9)$ \\
& & & & \\
& & & &
\end{tabular}

Only $55 \%$ female and $53 \%$ male respondents indicated they had undertaken a medical assessment for diving at least once. Twenty-nine per cent of females and 39\% of males had been examined by a physician trained to conduct assessments of fitness to dive. Twenty per cent of females $(n=60)$ and $31 \%$ of males $(n=111)$ went for repeated medical examinations. Of these, 23 females and 77 males were instructors, so were obliged to do so. Details about health issues and divingrelated diseases are given in Table 4.

Twenty per cent of females $(n=61)$ and $16 \%$ of males $(n=59)$ were on regular medications (Figure 2). The most commonly taken medication was thyroid hormone by the female divers $(6 \%, n=18)$. Anti-hypertensives (antiHT) were the most common taken by males $(3 \% ; n=12)$. Only $0.6 \%$ of female respondents took this type of drug; however, $3 \%$ of females $(n=9)$ and $9 \%$ of males $(n=34)$ reported 
taking medication associated with cardiovascular diseases (CVD); antiHT, lipid lowering agents and antiplatelet drugs. Of these 43 divers, only one female and seven males had been assessed by a dive physician. One female diver was on antiepileptic drugs and she had not received any medical advice.

Middle ear and/or sinus barotrauma were reported by eight females and 11 male divers. One male had a penetrating injury made by a sharp object during diving.

\section{DECOMPRESSION SICKNESS}

Five confirmed DCS cases were reported. One female diver had neurological DCS. One male was a commercial diver and developed DCS during a work dive, two males reported having neurological DCS about twenty years previously and one had had non-neurological DCS. None of these divers had any signs or symptoms of chronic disease or were on any medication, but the male divers were all overweight. Of the three females and 11 males who reported having had 'DCS-like' symptoms, one female and eight males had a $\mathrm{BMI} \geq 30$. Otherwise they were all healthy and none were on medication. Neither DCS nor reported DCS-like symptoms were statistically significantly different in occurrence between the sexes. The rate of physician-diagnosed DCS per 1,000 dives was 0.015 for females and 0.017 for males (n.s.). The rates of reported DCS-like symptoms per 1,000 dives also did not differ statistically between the sexes.

\section{DATA SPECIFIC TO FEMALES}

Twenty-one (7\%) female divers reported undergoing cosmetic surgery of some type, of which four were silicone implants. None reported having problems related to these surgeries during diving.

Ninety-nine per cent of female divers $(n=299)$ answered the questions about children. Twenty-one per cent $(n=62)$ were mothers, with a total of 72 children and one still birth. Fifty-one females had one child and 11 females had two children, none had three or more. Of the 72 children, 41 were boys and 31 were girls.

Sixty of the 62 mothers answered the question about their children's birth. Fifty-six of the first-born children were healthy and on time while four were premature. There was a history of diving during the first trimester for two of these premature births. All of the second children $(n=11)$ had a full-term birth. One hundred and two females replied to the question "did you dive during pregnancy" and seven stated that they dived in the first trimester. Of these seven pregnancies, one ended in a still birth and two ended with premature births (as mentioned above).

With regards to menstruation, 285 females answered the related questions. Of these, 90\% $(n=255)$ usually complained of pain or discomfort during menstruation.

\section{Figure 2}

Numbers of respondents by gender reporting regular medications; AntiHT - hypertension medication; LLA - lipid lowering agent; OAD - oral anti-diabetics; OCP - oral contraceptive pills (not all respondents answered the questions)

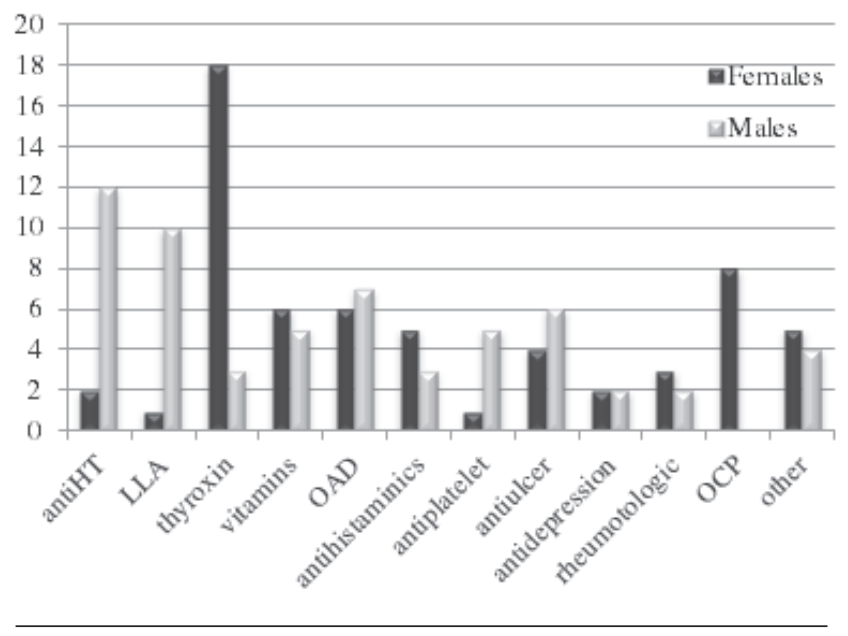

Regardless, 74\% ( $n=212)$ had dived during menstruation and did not encounter related problems. Of the 180 females who used tampons in daily life, $71 \%(n=127)$ also used them during diving and none reported experiencing a complication during or after diving. Nine female divers took oral contraceptive pills (OCP) and none of these divers had been diagnosed with decompression sickness (DCS) or reported experiencing DCS-like symptoms.

\section{Discussion}

Whilst there was a higher number of male than female respondents, this response rate is not completely representative of the proportion of male divers reported by Turkish national certification data $(79 \%)$, nor by global data (72\%). This may be owing to a male reluctance to complete surveys. ${ }^{6,12,13}$ Female respondents were significantly younger than the males, which is in general agreement with data from other countries. ${ }^{2,8,12}$ In comparison to data from a study conducted 10 years previously in Turkey, there was no significant change in the female divers' mean age $(P=0.13)$, but the mean BMI of the present group was significantly higher $(P=0.01) .{ }^{5}$ There are no previous data to compare these results to for male divers.

\section{DIVING HISTORY}

Our findings suggest that in the decade since our first study on female divers, the mean time elapsed since learning to dive had lengthened by three years and the mean number of dives had increased three-fold. ${ }^{5}$ It appears that Turkish female divers are now more actively involved in and continue diving after training. Despite this increase, males were still more experienced, with their mean time since learning to dive and the number of dives performed per year being significantly higher; $50 \%$ of female divers made less than 100 dives whereas the majority of males made over 100 . 
This probably reflects that $40 \%$ of males but only $15 \%$ of female respondents were diving instructors. This also reflects the data from other countries, where males have higher certifications and more dives per year. ${ }^{8,14}$

Whilst about two-thirds of divers reported making safety stops for all dives (including those not requiring decompression), only about half defined the nature of the stops used, e.g., at 3-5 msw for 3-5 minutes. Further research is needed, perhaps using depth-time loggers, before we can have a better understanding of the types of safety stops undertaken by recreational divers in Turkey.

Significantly more male than female divers performed decompression dives and did not adhere to recreational depth limits. It is not known whether this behaviour is related to overconfidence arising from experience or if it stems from cultural issues. However, many female divers also seem to violate recreational dive rules. This general noncompliance by divers in Turkey may well reflect an inadequacy in the training programmes offered by local diving schools. Unfortunately, 232 (32\%) of respondents did not answer this question, so this result may not be representative of the diver population as a whole. These findings are worthy of further study.

\section{HEALTH ISSUES}

Smoking was as prevalent among female divers as among male divers (35\% and 34\% respectively) but had declined from 10 years previously (42\%). This decrease seems promising, but smoking in divers is still greater than that observed in the general female population $(25 \%$ in 2002 , and $15 \%$ in 2010). ${ }^{15,16}$ Conversely, smoking among male divers was less prevalent than in the general male population $(48 \%) .{ }^{16}$ When compared with other diving populations, such as the UK and Australia, smoking amongst Turkish divers is much commoner. ${ }^{12,13}$ This is probably owing to increased awareness in other countries of the risks of smoking both for diving and generally.

Alcohol consumption among Turkish divers is not excessive and not very different from previously reported groups. ${ }^{12,13}$ However, a considerable number of divers, especially males, drink before diving despite evidence that diving under the influence of alcohol increases the risk of diving incidents. ${ }^{17}$ None of the questions asked if problems had occurred during a dive due to alcohol consumption beforehand.

The use of thyroid hormone in female divers is in line with that of the general Turkish population. ${ }^{18}$ Endemic iodine deficiency (ID) from lack of iodine in natural resources is a major health problem in Turkey; it causes impairment of thyroid hormone synthesis and so hypothyroidism, and is more common among females. Despite a mandatory salt iodization programme being initiated after severe ID was reported in the 1990s, severe to moderate ID persists in Turkey, along with a few other countries in the region. ${ }^{18,19}$
An inconsistency was evident between the cardiovascular diseases reported and cardiovascular medication used. Cardiac disease was reported by only 13 divers (five female and eight male), whereas 43 reported that they were taking cardiovascular medication. Similar usage is reported from other countries, but an adequate comparison with the general Turkish population could not be made as there are no clear national data available on cardiac diseases and dyslipidaemia or medication use. ${ }^{12,13}$ However, hypertension and diabetes are more comprehensively studied. The prevalence of HT in the general population for both women and men is $30 \%$, but in the present study, only $4 \%$ of male divers and fewer than $1 \%$ of female divers were reportedly taking anti-HT treatment. Even fewer reported having hypertension. These results are significantly lower than observed in the general Turkish population $(P<0.0001)$. Divers who reported being treated for diabetes were also less common than found in the general population $(14 \%) .^{20}$

One important implication identified was that Turkish divers may be at increased risk during diving due to a high prevalence of CVD and associated conditions. Divers Alert Network (DAN) report that a history of CVD and age greater than 40 years constitute one fourth of disabling injuries in diving fatalities. ${ }^{21}$ Fifty-five per cent of male Turkish divers were of age 35 or older and $10 \%$ were already on CVD medication. Additionally, smoking and obesity are factors known to increase the risk for cardiac incidents. ${ }^{22}$ In the present study, $34 \%$ of male respondents were smokers, $50 \%$ were overweight and $12 \%$ were obese or morbidly obese. In this regard, Turkish male divers seem to have an increased risk for cardiac injury.

Fitness to dive assessments were not commonly sought by Turkish divers. Evaluations made by a physician trained in diving medicine were even less common. Some people continue to dive with medical conditions that may have a negative impact on diving safety. Without more dive medicals, it cannot be established whether participants are fit to dive or not. Similar situations are reported from other countries where health clearance is based on selfdeclaration. ${ }^{12,13}$ It may be necessary to review the safety implications of the current system in Turkey.

\section{DECOMPRESSION SICKNESS}

There are few data about DCS occurrence in Turkish divers. In a previous study, DCS cases treated with recompression were analyzed but these were not specific to recreational diving. ${ }^{23}$ In a more recent Turkish study, of 132 recreational divers, DCS was reported by three $(2.2 \%){ }^{24}$ This was higher than that in our study $(0.8 \%)$. Further analysis of the relationship between DCS occurrence and factors like experience, diving depths and weight/BMI could not be carried out, as the group with confirmed DCS was too small.

There has been long-term discussion over the effect of sex, if any, on the occurrence of DCS. ${ }^{9}$ Recently, diving was shown 
to induce inflammatory changes which may have a role in DCS. ${ }^{25}$ There is cumulative evidence that inflammation pathways and cells work differently in females and males especially after exercise. ${ }^{26}$ Also, it is known that female hormonal cycles modulate endothelial functions that have been shown to be impaired by bubbles. ${ }^{25,27}$ Therefore, sex may still be a relevant issue for DCS. In this study, physiciandiagnosed DCS and DCS-like symptoms were slightly more prevalent in male divers than females but, given the small numbers, no inferences can be made from this survey. Nevertheless, our results are consistent with previous studies that have reported lower intravascular bubbles counts in females and a higher incidence of DCS in male divers. ${ }^{9,14,28}$

\section{DATA SPECIFIC TO FEMALES}

The mean age, maternity rate and number of children of female divers were consistent with the general Turkish population of the same socio-economic status. ${ }^{29}$ Whether diving influences an infant's sex has long been a speculative topic. However, we found no statistical difference between the proportion of male to female offspring born to Turkish diving mothers and that in the general Turkish population. ${ }^{30}$

Diving during pregnancy is not advised due to possible deleterious effects on foetal development. ${ }^{31}$ Of seven women who dived during the first trimester, one had a stillbirth and two babies were born prematurely. Once again, it is not possible to comment on the effect of diving while pregnant, given the small numbers in this survey.

Safety of diving during menstruation is a concern for many women. Mucosal congestion may predispose divers to barotrauma. ${ }^{7}$ Moreover, there are studies showing that the incidence of DCS may increase during the first phase of menstruation. ${ }^{27,32}$ However, the women in our study did not report any diving-related diseases while menstruating.

None of the female divers who were taking oral contraception (OCP) pills (OCP) reported any related problems. Interestingly, OCP use among the general Turkish female population $(6 \%)$ is twice that reported by the divers $(3 \%)$ in this study. ${ }^{33}$ This differs from European countries, where the rate of OCP use among divers is similar to or higher than the general female population (around 30\%). ${ }^{9,34,35}$ The low use of OCPs among Turkish females is not unexpected, and is explained by cultural differences; traditional contraceptive methods are commoner in Turkey. ${ }^{33}$ However, the fact that OCP use in divers is half that of the general population is surprising; this trend has only been reported in one other country (Australia; $9 \%$ in divers, $30 \%$ in the general poulation), but their uptake is still much higher than in Turkish female divers. ${ }^{12,35}$ It was thought that OCPs could heighten DCS risk and contraindicate diving because of an increased risk of thromboembolism. However, there is insufficient data to support this claim. ${ }^{7}$ Whether this anecdotal belief or cultural factors influenced the lower uptake of OCPs within the Turkish female diving population needs further investigation.

\section{LIMITATIONS}

This study has some limitations: mainly potential selection bias, limited numbers and incomplete survey responses. Most of the participants left one or two questions unanswered, thus there was a different number of respondents for every question, which might impair the consistency of the results. To avoid selection bias and confusion, the number of 'noresponse' answers for each question was also reported. Surveys of this type may also be subject to recall bias and verification of the data is not possible. Nevertheless, anonymous surveys may have benefits compared to face-toface surveys; the participants may be less likely to conceal medical conditions or dangerous diving practices, and so might provide less biased data.

\section{Conclusions}

This is the first large-scale recreational diving survey profiling female and male Turkish divers. Turkish divers are like those of other countries in terms of demographic composition, diving practices and medical conditions. Similarly, they continue diving with medical conditions that may contraindicate diving and most are not examined for fitness to dive. Consequently, dive organizations and local federations should probably consider regulating medical screening for divers. There are clear differences between Turkish female and male divers in their demographic characteristics, experience levels and diving behaviour. Safe diving practices seemed to be neglected by both genders, though more commonly by males. This neglect might indicate that the training standards of local dive schools, which are also relatively new to the industry, should be reviewed and regulated better. No statistical differences between females and males were found in the rates of confirmed DCS and DCS-like symptoms. However, it should be noted that this study did not evaluate susceptibility to DCS and does not rely on objective observations, but selfreported data only.

\section{References}

1 Sports and Fitness Industry Association. Participation in recreational diving report 2015. Silver Springs MD: SFIA, 2015. [cited in 2016 March 12]. Available from: https:// www.sfia.org/reports/331_Scuba-Diving-ParticipationReport-2015.

2 PADI Worldwide Corporate Statistics 2016. [cited 2017 May 18]. Available from: https://www.padi.com/sites/default/files/ documents/about-padi/statistics/PADI_2016_WW_Statistics. pdf.

3 Britısh Sub-Aqua Club annual diving incident reports for 2014 through 2016. [cited 2017 July 15]. Available from: https:// www.bsac.com/page.asp?section=1038.

4 Lippmann J, Lawrence C, Fock A, Jamieson S, Harris R. Provisional report on diving-related fatalities in Australian waters in 2011. Diving Hyperb Med. 2016;46:207-40.

5 Aktas S, Isıl S, Isler H. Türk kadın dalıcıları dalıs profili anketi. (Turkish female divers profile survey). Sualtı Bilim 
ve Teknolojisi Toplantısı Bildiriler Kitabı 1998 Aralık 12-13, [Underwater Sciences and Technology Meeting Proceedings 1998, Dec 12-13]; Istanbul: Istanbul Tip Fakültesi; 1998. p. 130-8. Turkish.

6 TSSF 2014 y1l aktivite raporu [Turkish Underwater Sports Federation 2014 activity report]. Istanbul; 2015. Turkish.

7 Edmonds C, Bennett M, Lippman J, Mitchell S, editors. Diving and subaquatic medicine, 5th ed. Boca Raton FL: CRC Press; 2015.

8 St Leger Dowse M, Bryson P, Gunby A, Fife W. Comparative data from 2250 male and female sports divers: diving patterns and decompression sickness. Aviat Space Environ Med. 2002;73:743-9.

9 Boussuges A, Retali G, Bodere-Melin M, Gardette B, Carturan D. Gender differences in circulating bubble production after SCUBA diving. Clin Physiol Funct Imaging. 2009;29:400-5.

10 TSSF. Donanımlı Dalıs Yönetmeligi. Resmi Gazete. Sayı: 26993. [Turkish Underwater Sports Federation, SCUBA diving regulations. Official gazete no. 26993]. 2008. [cited 2016 April 7]. Available from: http://www.resmigazete.gov. tr/eskiler/2008/09/20080910-10.htm. Turkish.

11 Profesyonel Sualtı Adamları Yönetmeligi. Resmi Gazete. Say1:23098. [Professional Diving Regualtions. Official gazete no 23098]. 1997. [cited 2016 March 12]. Available from: http://www.resmigazete.gov.tr/eskiler/2008/02/20080215-6. htm. Turkish.

12 Taylor DM, O’ Toole KS, Ryan CM. Experienced, recreational scuba divers in Australia continue to dive despite medical contraindications. Wilderness Environ Med. 2002;13:187-93.

13 St Leger Dowse M, Waterman MK, Penny CE, Smerdon GR. Does self-certification reflect the cardiac health of UK sport divers? Diving Hyperb Med. 2015;45:184-9.

14 Ranapurwala SI, Bird N, Vaithiyanathan P, Denoble PJ. Scuba diving injuries among Divers Alert Network members 20102011. Diving Hyperb Med. 2014;44:79-85.

15 Emri S, Basoglu A, Turnagöl H, et al. Epidemiology of smoking among Turkish adults: a national household survey, 2002. The Second International Symposium on Medical Geology, Nutrition, and Cancer, March 31-April 03, 2003. Istanbul, Turkey: Military Cultural Center; 2003. p. 33-6.

16 Global Adult Tobacco Survey, Turkey Report, 2010, In: Primary Health Care General Directorate. Ministry of Health publication number: 803. Ankara: Anıl Press; 2010.

17 Davis M. The Editor's offering. Alcohol and scuba diving. Diving Hyperb Med. 2012;42:193.

18 Erdogan MF, Agbaht K, Altunsu T, Ozbas S, Yucesan F, Tezel $\mathrm{B}$, et al. Current iodine status in Turkey. J Endocrinol Invest. 2009;32:617-22.

19 WHO Nutrition. The WHO Global Database on Iodine Deficiency. 2008. [cited 2017 May 18]. Available from: http:// www.who.int/vmnis/en/.

20 Satman I, Omer B, Tutuncu Y, Kalaca S, Gedik S, Dinccag N, et al. Twelve-year trends in the prevalence and risk factors of diabetes and prediabetes in Turkish adults. Eur J Epidemiol. 2013;28:169-80.

21 Mitchell SJ, Bove AA. Medical screening of recreational divers for cardiovascular disease: consensus discussion at the Divers Alert Network Fatality Workshop. Undersea Hyperb Med. 2011;38:289-96.

22 Pougnet R, Di Constanzo L, Lodde B, Henckes A, Dherbecourt $\mathrm{L}$, Lucas D, et al. Cardiovascular risk factors and cardiovascular risk assessment in professional divers. Int Marit Health. 2012;63:164-9.

23 Toklu AS, Cimsit M, Yildiz S, Uzun G, Korpinar S, Sezer H, et al. Decompression sickness cases treated with recompression therapy between 1963 and 1998 in Turkey: review of 179 cases. Undersea Hyperb Med. 2014;41:217-21.

24 Ozdemir L, Duru-Asiret G, Bayrak-Kahraman B, Devrez N, Akbayir A. Health-related adverse events and associated factors in recreational divers with different certification levels. J Travel Med. 2013;20:289-95.

25 Lambrechts K, Balestra C, Theron M, Henckes A, Galinat H, Mignant $\mathrm{F}$ et al. Venous gas emboli are involved in post-dive macro, but not microvascular dysfunction. Eur J Appl Physiol. 2017; 117:335-44.

26 Abbasi A, de Paula Vieira R, Bischof F, Walter M, Movassaghi $\mathrm{M}$, Berchtold NC et al. Sex-specific variation in signaling pathways and gene expression patterns in human leukocytes in response to endotoxin and exercise. J Neuroinflammation. 2016;13:289.

27 St Leger Dowse M, Gunby A, Phil D, Moncad R, Fife C, Morsman J, et al. Problems associated with scuba diving are not evenly distributed across a menstrual cycle. J Obstet Gynaecol. 2006;26:216-21.

28 Klingmann C, Gonnermann A, Dreyhaupt J, Vent J, Praetorius M, Plinkert PK. Decompression illness reported in a survey of 429 recreational divers. Aviat Space Environ Med. 2008;79:123-8.

29 TR Ministry of Development and TUBITAK. The 2013 Turkey Demographic and Health Survey. In: Hacettepe University Institute of Population Studies. Ankara: Elma Teknik Basım Matbaac1lık Ltd; 2014.

302014 Birth statistics. TUIK news bulletin no:18621. Turkish Statistical Insititute; 2015. [cited in 2016 March 15]. Available from: http://www.turkstat.gov.tr/PreHaberBultenleri. do?id=18621.

31 Conger J, Magann EF. Diving and pregnancy: what do we really know? Obstet Gynecol Surv. 2014;69:551-6.

32 Lee VM, St. Leger Dowse MS, Edge C, Gunby A, Bryson P. Decompression sickness in women: a possible relationship with the menstrual cycle. Aviat Space Environ Med. 2003;74:1177-82.

33 [Turkish oral contraception guide]. (Türkiye Oral Kontrasepsiyon Kilavuzu). Istanbul: Cortex Iletisim Hizmetleri AS; 2012. Turkish

34 St Leger Dowse M, Gunby A, Moncad R, Fife C, Smerdon G, Bryson P. The combined oral contraceptive_pill and the assumed 28-day cycle. J Obstet Gynaecol. 2007;27:481-4.

35 United Nations. World contraceptive pattern 2013. [cited 2017 May 18]. Available from: http://www.un.org/en/development/ desa/population/publications/pdf/family/worldContraceptive PatternsWallChart2013.pdf.

\section{Acknowledgement}

The authors would like to thank Peter Buzzacott for his invaluable help in developing and improving this manuscript.

\section{Funding and conflicts of interest: nil}

Submitted: 11 May 2016; revised 24 February and 13 June 2017 Accepted: 22 July 2017 


\title{
Diving with pre-existing medical conditions John Lippmann ${ }^{1,2}$, David McD Taylor ${ }^{3,4}$, Christopher Stevenson², Jo Williams², Simon J Mitchell ${ }^{5}$
}

\author{
${ }^{1}$ Divers Alert Network Asia-Pacific, Ashburton, Victoria, Australia \\ ${ }^{2}$ School of Health and Social Development, Deakin University, Victoria, Australia \\ ${ }^{3}$ Emergency Department, Austin Hospital, Victoria, Australia \\ ${ }^{4}$ Department of Medicine, University of Melbourne, Australia \\ ${ }^{5}$ Department of Anaesthesiology, University of Auckland, Auckland, New Zealand
}

Corresponding author: John Lippmann, DAN Asia Pacific, PO Box 384, VIC 3147, Australia johnl@danap.org

\section{Key words}

Fitness to dive; Scuba divers; Cardiovascular; Diabetes; Respiratory; Asthma; Survey

\begin{abstract}
(Lippmann J, Taylor D McD, Stevenson C, Williams J, Mitchell SJ. Diving with pre-existing medical conditions. Diving and Hyperbaric Medicine. 2017 September;47(3):180-190.)

Introduction: This is the second report based on a survey of Divers Alert Network Asia-Pacific (DAN AP) members who dive with cardiovascular and respiratory conditions and diabetes. It examines the medical management of the divers' conditions, any diving modifications used to mitigate the risk and outcomes.

Methodology: An online cross-sectional survey was sent to 833 divers who had declared a targeted medical condition when applying for DAN AP membership between July 2009 and August 2013.

Results: Two-hundred-and-sixty-eight respondents (32\%) provided sufficient information on their conditions to be included in the analyses. These included ischaemic heart disease (31), arrhythmias (20), cardiac septal defects (31), other cardiac conditions (10), hypertension (127), diabetes (25), asthma (40) and pneumothorax (5). Forty-nine per cent had sought specialist diving medical advice about their condition and $23 \%$ reported modifying their diving practices to mitigate their risk. The cohort had completed 183,069 career dives, 57,822 of these since being diagnosed with their medical condition. There were 27 individuals who reported having decompression illness ( 25 of whom were subsequently diagnosed with a persistent foramen ovale), and two individuals who experienced an arrhythmia during diving.

Conclusions: Some DAN AP members are diving with medical conditions which could potentially impact the safety of their diving. A minority modified their diving practices to mitigate the risk of their condition and approximately half sought specialist diving medical advice. The incidence of diving-related problems precipitated by known and managed pre-existing health conditions seems low but further studies of larger cohorts and incorporating fatality data would be necessary to confirm this. These results are limited by the $32 \%$ response rate and potential for bias towards selection of those most careful with their health.
\end{abstract}

\section{Introduction}

Medical conditions such as asthma, diabetes and many cardiac conditions were long considered absolute contraindications to scuba diving. ${ }^{1-3}$ Some sufferers have ignored such advice and, over time, diving medical organisations have progressively modified their advisories on diving with conditions such as asthma and diabetes to allow candidates meeting certain criteria to dive. As a result, it seems likely that an increasing number of divers with these and other comorbidities, including a variety of cardiovascular conditions, are diving. This has created an increasing need to learn more about the medical conditions of active divers, how these are managed and the impact, if any, that these conditions may have on diving practices and experiences.

This is the second report from a study investigating Divers Alert Network Asia-Pacific (DAN AP) members with declared cardiovascular and respiratory conditions and diabetes (targeted conditions). The first report provided information about the demographics, diving history and activity of members with the targeted conditions and compared these to members without these conditions. ${ }^{4}$ This article more closely examines the medical management of the divers' conditions, and how these divers modify their diving practices (if at all) in an attempt to mitigate the risk of a diving incident consequent upon their medical condition.

\section{Methods}

Ethics approvals were received from the Human Research Ethics Committees of Austin Health and Deakin University, both in Victoria, Australia. An anonymous, online, crosssectional medical conditions survey (MCS) was conducted on a cohort of 833 DAN AP adult (> 18 years old) members. The divers had joined DAN AP between 01 July 
2009 and 01 August 2013 and had, at the time of joining, declared that they suffered from hypertension, diabetes, respiratory or cardiac conditions. The cardiac conditions mainly comprised ischaemic heart disease (IHD) (previous myocardial infarction and angina), arrhythmias, and septal defects (i.e., persistent foramen ovale (PFO) and atrial and ventricular septal defects). These self-reported diagnoses were interpreted at face value.

An invitation to participate in the survey was sent to these members in August 2013, a reminder was sent in October 2013 and the survey was closed in December 2013. No inducements to participation were offered. Invitees were assured of their anonymity and that responding or otherwise would have no impact on their insurance status.

The MCS consisted of a two-part questionnaire. The first part sought details of the respondents' demographics and diving activity. The second part focused on their targeted medical condition and its management. There were also specific questions about any impact these conditions had on the respondents' diving practices and any adverse incidents that had occurred. The questionnaires are available at $<\mathrm{http}: / /$ www.danap.org/research/med_conditions/>.

All invitees were able to access the first part of the questionnaire, as well as the set of questions relating to their declared condition(s). Participants were invited to enter their responses directly into an online, dedicated, structured query language (MySQL) database (Oracle, Redwood CA).

\section{STATISTICAL ANALYSIS}

Responses were downloaded into MS Excel ${ }^{\circledR}$ (Microsoft Corporation, Redmond WA) for collation. Respondents reporting more than one relevant condition (e.g., hypertension and diabetes) were included in the analysis for each relevant condition. A descriptive analysis based on means and standard deviations or median and ranges as appropriate was conducted using SPSS Version 22 (IBM, Armonk, NY; 2013). A conservative minimum required sample size was calculated using the NSS online calculator, ${ }^{5}$ assuming a proportion with any specific characteristic of 0.5 and a confidence interval of \pm 0.05 . The minimum sample size required was calculated to be 263 .

\section{Results}

Three-hundred-and-forty-three of 833 divers (41.2\%) who had reported a targeted medical condition in their DAN membership application responded to the questionnaire. Two hundred and sixty-eight (32.2\% of invitees) of the respondents $(78.1 \%)$ provided sufficient information for inclusion in the study. The mean (SD) age of the invitees was 50 (12) years and $73 \%$ were males. By comparison, the mean (SD) age of the respondent cohort was 52.4 (12) years and the proportion of males was 70\%. Twenty-one respondents had multiple conditions. Males were in the majority for all diagnostic subgroups except septal defects (10 of 31 male). The mean (SD) age for males was 53.5 (11.8) years, and for females 48.7 (12.4) years (Table 1). The overall mean (SD) body mass index (BMI) was 27 (4) kg. $\mathrm{m}^{-2}$. Males had a mean (SD) BMI of 28 (4) kg.m ${ }^{-2}$ and females 26 (4) kg.m ${ }^{-2}$.

The medical conditions of interest were cardiac conditions (92 cases), hypertension (127), diabetes (25) and respiratory conditions, predominantly asthma (45).

Overall, these divers reported a median (interquartile range, IQR) of $350(150,800)$ total dives over a median time of $12(6,22)$ years, with a median of $150(60,350)$ dives done post-diagnosis. Males had dived much more frequently than females (median total dives of 1,000 $(388,2,125)$ and 400 $(225,1,000)$ respectively). Details of their diving histories and activities are reported in Table 2 . These 268 divers had completed 183,069 career dives including 57,822 since being diagnosed with their medical condition. Other than 27 individuals with decompression illness (DCI) (25 of whom were subsequently diagnosed with a PFO) and two cases of arrhythmias (see later), they reported no other relevant adverse events from diving.

\section{Table 1}

Demographic information of 268 individual divers with 289 targeted medical conditions (some with multiple conditions); BMI - body mass index; IHD - ischaemic heart disease; N/A - not applicable; * single case; mean (SD) shown for age and BMI

\begin{tabular}{|c|c|c|c|c|c|c|c|c|c|}
\hline \multirow[b]{2}{*}{ Group } & \multicolumn{7}{|c|}{ Medical condition } & \multirow[b]{2}{*}{$\begin{array}{l}\text { Pneumothorax } \\
(n=5)\end{array}$} & \multirow[b]{2}{*}{$\begin{array}{l}\text { Total } \\
(n=289)\end{array}$} \\
\hline & $\begin{array}{c}\text { IHD } \\
(n=31)\end{array}$ & $\begin{array}{l}\text { Arrhythmia } \\
\quad(n=20)\end{array}$ & $\begin{array}{c}\text { Septal defect } \\
\quad(n=31)\end{array}$ & $\begin{array}{l}\text { t Cardiac } \\
(\text { other, } n=10)\end{array}$ & $\begin{array}{c}\text { Hypertension } \\
(n=127)\end{array}$ & $\begin{array}{c}\text { Diabetes } \\
(n=25)\end{array}$ & $\begin{array}{l}\text { Asthma } \\
(n=40)\end{array}$ & & \\
\hline \multicolumn{10}{|c|}{ 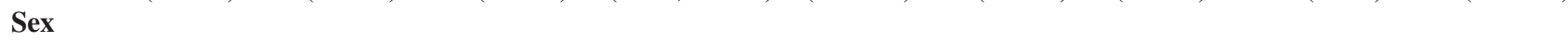 } \\
\hline $\mathrm{M} / \mathrm{F}$ & $28 / 3$ & $16 / 4$ & $10 / 21$ & $6 / 4$ & & $19 / 6$ & $24 / 16$ & $5 / 0$ & 187/102 \\
\hline \multicolumn{10}{|l|}{ Age (yrs) } \\
\hline All & $59.1(7.2)$ & $0.1(13.6)$ & $45.5(10.7)$ & $47.3(11.7)$ & & $54 \quad(11)$ & & & $52 \quad(12)$ \\
\hline Male & $58.3(6.7)$ & $63.0 \quad(8.8)$ & $49.2 \quad(8.9)$ & $46.8(14.1)$ & & $56.1(10.1)$ & $40.5(1$ & $48.4(12.1)$ & $53.5(11.8)$ \\
\hline Female & $71.0(0)^{*}$ & $43.5(18.2)$ & $46.6(11.0)$ & $48.0 \quad(8.7)$ & & $53.8 \quad(9.2)$ & $40.0(10.1)$ & N/A & $48.7(12.4$ \\
\hline \multicolumn{10}{|c|}{ BMI $\left(\mathrm{kg} \cdot \mathrm{m}^{-2}\right)$} \\
\hline All & $29.1(4.2)$ & $25.8 \quad(4.2)$ & 25.6 & $24.7 \quad(4.9)$ & & $29.4 \quad(5.1)$ & $25.8 \quad(3.7)$ & $27.0 \quad(2.2)$ & $27.3 \quad(4.2$ \\
\hline Male & $29.3(4.1)$ & $26.5(4.2)$ & $27.8 \quad(3.0)$ & $24.2 \quad(3.8)$ & & $29.7 \quad(4.8)$ & 26.4 (4.3) & $27.0 \quad(2.2)$ & 28.0 \\
\hline Female & $23.8(0)^{*}$ & 22.1 & $24.5 \quad(3.4)$ & $25.2(6.9)$ & $26.6(3.5)$ & $28.3 \quad(6.5)$ & $25.0 \quad(3.0)$ & N/A & $25.5 \quad(3.8$ \\
\hline
\end{tabular}




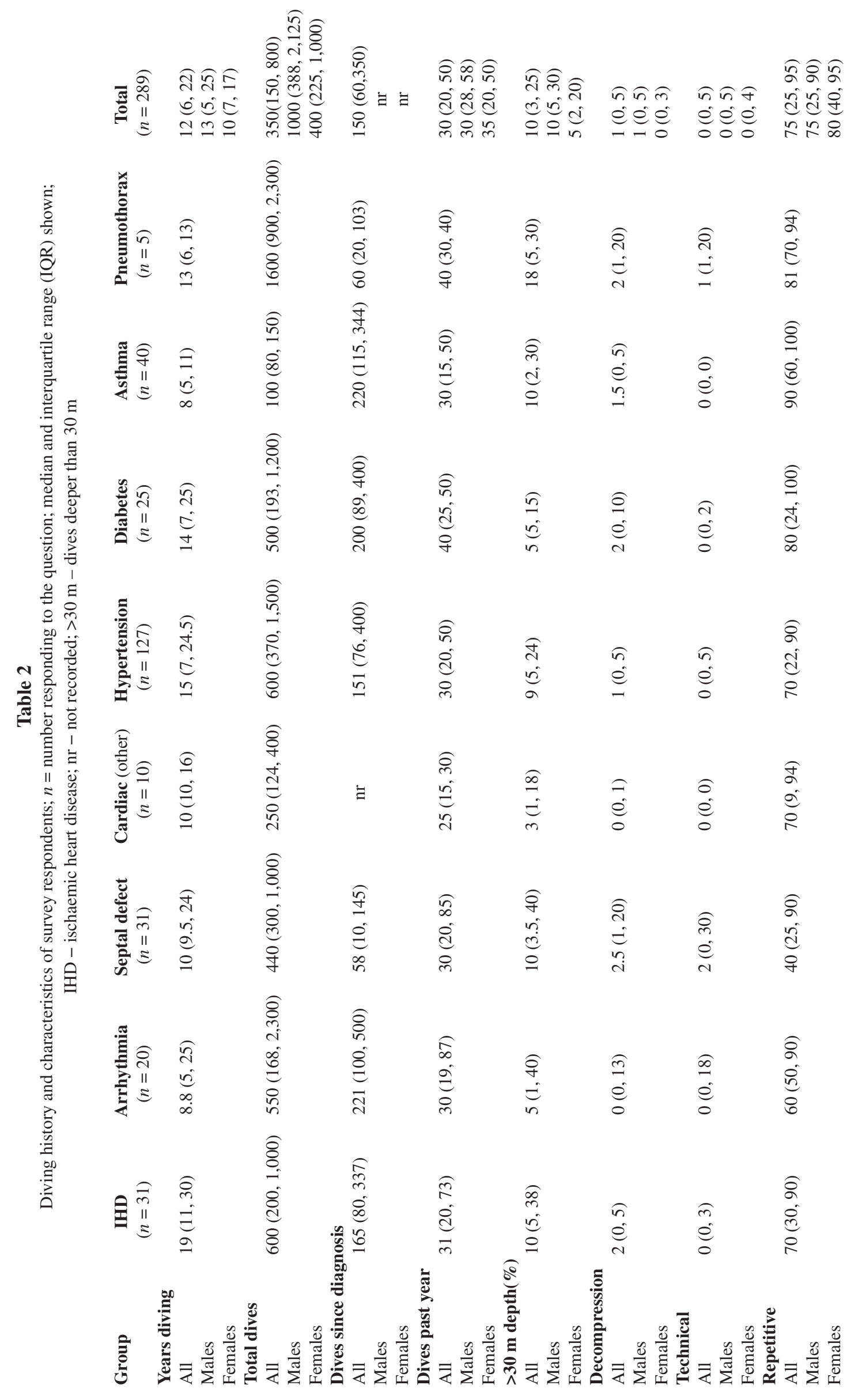




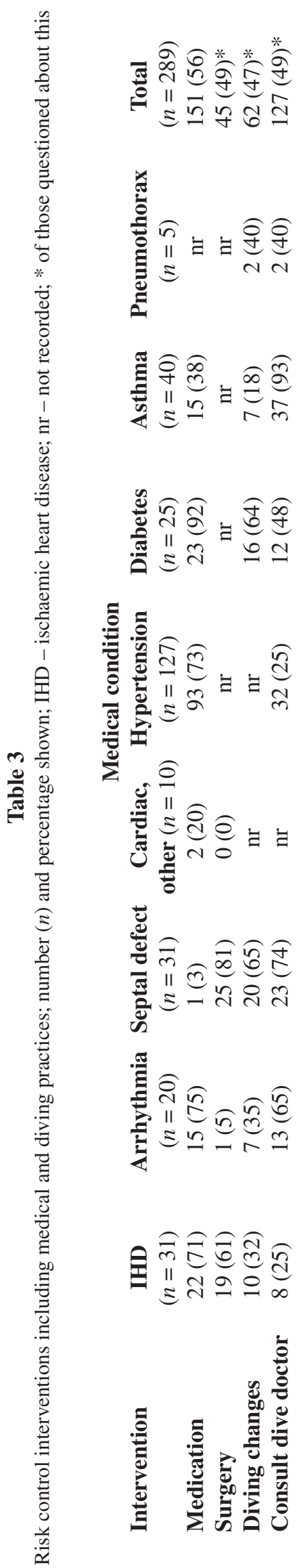

\section{INTERVENTIONS}

Table 3 shows relevant interventions, where reported, categorized as medication, surgery, changes in diving practice, and diving medical consultation. Specific interventions and activities are discussed by condition in the commentary below.

\section{ISCHAEMIC HEART DISEASE (IHD)}

These were divers who reported a previous myocardial infarction (MI) (22 divers), angina (10), "coronary stent" (without reference to the indicating symptoms or event) (2), "coronary artery bypass graft" (again, without reference to the indicating symptoms or event) (1), and "partially occluded coronary artery" (without explanation of the indication for an angiogram) (1). Fifteen of the 31 respondents with IHD reported co-morbidities of hypertension, diabetes and/or arrhythmia. Corrective interventions included stenting (13) and coronary artery bypass (6). The median (IQR) time since intervention was $5(2.5,8)$ years. These 19 respondents reported having done a total of 8,622 dives since intervention (including one male instructor and technical diver with 4,000 dives) with a median (IQR) of $100(60,250)$ dives. Relevant medications included statins (18), antihypertensives (14), antiplatelet agents (9), beta blockers (6) and anticoagulants (1). No respondents reported current chest pain or precipitation of cardiac-related symptoms during diving.

\section{ARRHYTHMIA}

Reported arrhythmias included atrial fibrillation (AF) (9 divers), ventricular tachycardia (3), supraventricular tachycardia (SVT) (3), first degree heart block (1), unspecified heart block (1) and unifocal ventricular ectopics (1). Two conditions were unspecified. One respondent (a male with Advanced and Enriched air certifications) had corrective surgery, an ablation for SVT, and had subsequently done more than 700 incident-free dives. Relevant medications included beta blockers (7), anticoagulants (2) and antiplatelet agents (2). One diver reported taking the antiarrhythmic flecainide.

Two respondents reported having experienced symptoms from their arrhythmia during diving. One of these, diagnosed with SVT, had two SVT-type events while exerting himself on the surface post-diving. The other, diagnosed with AF which was generally well-controlled, went into AF during a dive. On another occasion, this diver had an unrelated episode of "very mild" DCI (symptoms unspecified).

\section{SEPTAL DEFECTS}

Of the 31 respondents with a septal defect, 27 reported a PFO, three an atrial septal defect (ASD) and one a ventricular septal defect (VSD). The median (IQR) number of dives conducted prior to diagnosis of a septal defect was $438(252,763)$. The relative proportions of technical and decompression diving among respondents with known septal defects was higher than for the remainder of the cohort, albeit still generally low.

The total years' diving were similar for divers with a PFO or an ASD $(10(7.8,18)$ versus $9(7,18.5)$ respectively) . However, divers with a PFO had done more dives than those with an ASD before diagnosis; 400 
$(224,775)$ vs. $240(140,345)$ respectively. The diver with a VSD had been diagnosed prior to beginning to dive, had no intervention and had done 130 uneventful dives at the time of this survey. Twenty-five of the 31 respondents with septal defects had suffered DCI prior to discovery of their defect which is unsurprising because it is DCI events that typically provoke investigation for such lesions. These accounted for 25 of the 27 respondents with medical conditions who reported having DCI over the course of their diving careers.

Twenty-five of the 31 respondents underwent surgery to insert a closure device and, of these, 22 reported an effectively-closed shunt. These 25 respondents who underwent closure had undertaken 15,849 dives prior to the intervention with 23 individuals suffering DCI. After closure, they had completed 2,771 dives with only one individual (who was among the 22 who reported an effectively closed shunt) having a recurrence of DCI (several episodes of skin rash only). This individual exhibited no residual shunt in his post-procedure echocardiogram and has completed over 1,000 dives since.

One respondent reported taking long-term aspirin since his PFO closure. Medications taken by three others were unrelated to their septal condition.

\section{OTHER CARDIAC CONDITIONS}

Ten respondents reported having "other cardiac conditions" but only seven specified the condition (mitral valve prolapse (6) and heart murmur (1)). None of these seven reported taking medications, but one of the others was taking an anti-hypertensive agent.

\section{HYPERTENSION}

One-hundred-and-twenty-seven respondents reported having hypertension; the median (IQR) time since diagnosis of hypertension being $7(4.5,15)$ years. The main medication types reported included angiotensin II antagonists (43, $34 \%$ ), ACE inhibitors $(41,32 \%)$, calcium channel blockers $(29,23 \%)$ and $\beta$ blockers $(13,10 \%)$. The respondents with declared hypertension were asked to report their last blood pressure measurement, although it was not specified if this was measured by a healthcare professional or themselves. The mean (SD) reported systolic BP was 128 (9) and diastolic 78 (7).

Only one diver reported a general on-going problem associated with hypertension (headache) and none reported problems with diving as a result of their condition. One diver with hypertension also reported having had DCI but did not specify his symptoms. Perceived risks of diving with hypertension reported by the divers included cardiac events, stroke and decompression illness.

\section{DIABETES}

Twenty-five respondents reported diabetes. The mean (SD) BMI was 29 (5) $\mathrm{kg} \cdot \mathrm{m}^{-2}$ with four of normal weight, 13 overweight and eight obese. The median (IQR) time since diagnosis was $7.5(6,11.5)$ years and the total number (range) of dives conducted since diagnosis was 9,143 (30 to 3,500) dives. Four divers were insulin-dependent, 19 were controlled by oral medications plus diet and exercise, and two by diet and exercise alone. Medications reported were biguanides (19), sulfonylureas (6), insulin (4), dipeptidyl peptidase-4 inhibitors (4), alpha glucosidase inhibitor (1), meglitinide (1) and thiazolidinedione (1).

Only one respondent reported having ever been admitted to hospital as a result of their diabetes. Seven divers reported having self-managed a hypoglycaemic event ('hypo') in any context (none while diving), two of these having done so in the previous year. No respondent required the help of another person to manage their symptoms during the previous year. Of those who reported having a 'hypo', the median (IQR) time since the last 'hypo' was $2(1.25,2)$ years. Three respondents reported diabetes-related complications. These were cardiac, kidney disease and visual problems. Thirteen of the 25 had undergone an exercise ECG as part of their assessment.

Sixteen of the 25 diabetic respondents (including three of the four who were insulin-dependent) measured their blood glucose level (BGL) before diving, although none practiced strictly in accordance with the relevant guidelines of the South Pacific Underwater Medicine Society (SPUMS) or Undersea and Hyperbaric Medicine Society (UHMS). ${ }^{6,7}$ The frequency of pre-dive measurement varied from one to three times, and the timing from 15 minutes to three hours before diving. The mean (SD) minimum BGL that these respondents reported to be acceptable before diving was $6.1(1.6) \mathrm{mmol} \cdot \mathrm{L}^{-1}$ with a range of 3.5 to $10 \mathrm{mmol} \cdot \mathrm{L}^{-1}$. Five respondents changed their medication regimen prior to diving - three did not take their oral medications and two insulin-dependent divers reduced their insulin dose. Four respondents reported changing their mealtime to increase carbohydrate and sugar intake before diving. Only six respondents routinely checked their BGL post-diving (including two of the four insulin-dependent divers), only one of these doing so more than once. One insulin-dependent respondent reported checking his BGL hourly for three hours, while the other five respondents who routinely checked did so at periods of five minutes to several hours after diving.

Sixteen respondents reported being aware of the relevant guidelines and 10 of these indicated that these guidelines had influenced their diving practice. This included BGL monitoring, increased vigilance, greater medical oversight and careful buddy selection. Nine of the 12 respondents who consulted a diving doctor had periodic diving medical 
reviews, at intervals of 6-12 months. Perceived risks reported by the diabetics included loss of consciousness in- or underwater, confusion and an increased risk of decompression illness.

\section{ASTHMA}

Forty respondents reported asthma. Twenty-eight of 30 who answered the relevant question reported having been diagnosed with asthma before taking up diving. The median (IQR) time since diagnosis of asthma was $19(13,30)$ years. Eleven respondents had been admitted to hospital between one and four times, with the median (IQR) number of admissions being $1(1,2)$. The median (IQR) time since last admission was $16(15,23)$ years. The time since last asthma symptoms ranged from one week to 20 years with a median (IQR) of $2(0.3,10)$ years. Fifteen respondents had symptoms during the previous year including wheeze (13), reduced exercise capacity (9), dyspnoea (5) and increased sputum production (3). Identified asthma triggers were allergy (13), infection (8), cold (5) and stress (1). Thirteen respondents used 'preventer' medications, most often daily and 22 used bronchodilators (in all cases salbutamol) either daily (5), monthly (5) or annually (12). There were no reported exacerbations of asthma while diving. The perceived risks from asthma reported by the divers included pulmonary barotrauma, dyspnoea and reduced exercise capacity.

\section{PNEUMOTHORAX AND CHEST SURGERY}

Five respondents reported having had a pneumothorax one to five years previously. All were divers at the time of their event. Few condition-specific data were provided by two of the divers so the following is based on the three others. All were left-sided; two were trauma-related and were managed with a chest drain. The other was spontaneous and required no treatment. The two with trauma-related injuries underwent subsequent CT scans (one specifically for informing future diving) while the respondent with the spontaneous event did not. Subsequently the spontaneous pneumothorax victim had conducted 20 dives, whilst the two traumatic pneumothorax victims had completed 30 and 200 dives with no reported problems. Another respondent had a pulmonary lobectomy to remove cancer 11 years prior. He underwent a subsequent CT scan and returned to diving after consultation with a diving doctor. He subsequently had done 750 incident-free dives.

\section{MEDICATIONS}

One-hundred-and-fifty-one respondents reported taking a total of 337 medications. The main classes of medications taken are shown in Table 4. The full list of individual drugs is available on request from the corresponding author at $<$ johnl@danap.org>.

\section{Table 4}

Classes of medications from 151 respondents (some used multiple medications); * \% based on 337 reported drugs used by 151 respondents; \# includes two respondents on clopidogrel; table does not include medications taken by less than three respondents

\begin{tabular}{lcc} 
Class & Frequency & $(\%)^{*}$ \\
Statins & 51 & $(15)$ \\
Angiotensin 2 receptor antagonists & 43 & $(13)$ \\
ACE inhibitors & 41 & $(12)$ \\
Calcium channel blockers & 29 & $(9)$ \\
Bronchodilators & 22 & $(7)$ \\
Biguanides & 19 & $(6)$ \\
Diuretics & 17 & $(5)$ \\
Antiplatelet agents\# & 16 & $(5)$ \\
B blockers & 15 & $(4)$ \\
Inhaled glucocorticoids & 13 & $(4)$ \\
Proton pump inhibitors & 12 & $(4)$ \\
Sulfonylureas & 6 & $(2)$ \\
Insulin replacement & 4 & $(1)$ \\
Dipeptidyl peptidase-4 inhibitor & 4 & $(1)$ \\
serotonin reuptake inhibitors & 3 & $(1)$ \\
Serotonin-norepinephrine reuptake & & \\
inhibitors & 3 & $(1)$ \\
Non-steroidal anti-inflammatories & 3 & $(1)$ \\
Anticoagulants & 3 & $(1)$ \\
Thyroxine replacement & 3 & $(1)$ \\
Total & 307 & $(91)$ \\
& & \\
\hline
\end{tabular}

\section{DIVING MODIFICATIONS}

Sixty-two $(23 \%)$ respondents reported having directly modified their diving practice. It is possible that this is an under-estimate as many failed to respond to this question. Reported modifications included:

- $\quad$ exertion avoidance (MI, angina, arrhythmia);

- $\quad$ stress avoidance (MI, angina, asthma);

- $\quad$ reduced depth (MI, angina, septal defect );

- use of nitrox, more conservative dive profiles, padding decompression time or safety stops to be longer than prescribed by tables or computers, longer surface intervals (septal defects);

- less repetitive diving, especially true of the respondents with septal defects who engaged in a much lower proportion of repetitive dives than most of the other groups;

- careful buddy selection and briefing (diabetes, arrhythmia);

- $\quad$ avoidance of cold water (arrhythmia, asthma);

- reduced ascent rate (septal defects, pneumothorax).

In addition to the above actual diving modifications, some respondents also reported:

- $\quad$ increasing vigilance for symptoms before, during and after diving (arrhythmia, diabetes, asthma);

- $\quad$ adjustment of their medication (diabetes, asthma). 


\section{Discussion}

This study identified the characteristics, behaviours and outcomes of active divers with significant medical conditions. Despite our focus on some conditions that are perceived to significantly impact on diver safety, and setting aside the 25 cases of DCI occurring in divers with unrepaired septal defects, our respondents reported only five other significant adverse events in over 183,000 career dives, 57,000 of which occurred post-diagnosis and 13,000 over the year immediately preceding completion of the questionnaire. This is not surprising as, in many cases, the conditions had been or were being appropriately managed (e.g., closure of septal defects, coronary stenting, coronary bypass grafting, effective glucose control in diabetes) and that specific risk mitigation measures had sometimes been introduced to their diving practice. Although our numbers are small and these voluntarily reported data deserve cautious interpretation, our findings are generally supportive of an argument that these diagnoses per se are not necessarily contraindications to diving as they have frequently been considered in the past.

\section{ASTHMA}

Traditionally one of the greatest controversies in fitness to dive discussions involved asthma which, for a long time, was regarded as a complete contraindication to diving. Common triggers associated with the diving environment include exercise, cold, the breathing of cold, dry air, anxiety and salt water aspiration. An exacerbation while diving creates the potential for gas trapping and pulmonary barotrauma, as well as the potential for drowning as a result of dyspnoea.

Various surveys of diving cohorts in Australia, the United Kingdom (UK), the United States (USA) and of Germanspeaking divers have indicated a prevalence of active asthma in approximately $3-9 \%$ of respondents., ${ }^{4-12}$ There have been reports of pulmonary barotrauma and arterial gas embolism in asthmatics, ${ }^{8,13}$ but the extent to which asthma materially increases the risk of barotrauma remains unclear. A survey of DAN America members ${ }^{13}$ appeared to indicate an increased risk of DCI in those with asthma, while a U.K. report ${ }^{8}$ did not. Our respondents diagnosed with 'asthma' reported no related problems in 7,613 dives. Given the small numbers it is difficult to categorise this as anything other than 'reassuring', but like others, we see no signal of increased risk in our data.

Nevertheless, there are sufficient grounds for believing the risk is more than merely theoretical. A prospective study of 100 UK divers reported that 20 who would have been excluded, based on prevailing selection criteria, ${ }^{14}$ had problems during diving including wheezing underwater. ${ }^{15}$ Moreover, there are reports of diving fatalities that were directly attributed to asthma. ${ }^{16,17}$ The absence of related problems in ours and other's data may indicate that the risks of diving, particularly in mild asthmatics, is small or that most divers manage their asthma and diving activities adequately.

In our cohort of 40 divers, 37 had consulted a diving physician about their asthma. This would have allowed both optimisation of their condition and discussion of how best to manage it in relation to diving. Various diving medical guidelines are available (including those of SPUMS ${ }^{18}$ ) to guide both the physician and the diver in this setting, and all divers with asthma are well-advised to have a diving medical assessment by a doctor with relevant training.

\section{DIABETES}

Historically, diabetes mellitus, especially in its insulindependent form, has been considered an absolute contraindication to diving. The main concern is the consequences of reduced mentation or unconsciousness because of hypoglycaemia during diving. Blood glucose levels (BGLs) do fall with diving and such falls could be substantial and potentially dangerous under demanding conditions. Other concerns include diabetes-related endorgan damage, especially co-existing cardiac disease.

However, in 1991, the UK Sports Diving Medical Committee softened its position and the British Sub-Aqua Club (BSAC) and some other UK-based certification agencies led the way in enabling selected diabetic divers to dive, albeit with some restrictions. Several studies have subsequently been conducted to monitor the habits and blood glucose levels of divers with diabetes before, during and after real or simulated diving exposures. ${ }^{19-23}$ The accumulation of these data, with an associated low incidence of related problems, suggests that people with diabetes can dive safely provided risks are managed effectively by appropriately educated individuals.

As a result, the prohibition on diving with diabetes has eased and more individuals with diabetes are diving, preferably under the general oversight of their endocrinologist as well as a diving physician. In a 2000 survey of 346 experienced Australian divers, only one respondent $(0.2 \%)$ reported having diabetes. ${ }^{10}$ However, in 2014-15, 21/1,119 (1.9\%) DAN AP members, 8/350 (2\%) PADI members and 9/806 (1\%) of PADI-certified divers (most of whom did Open Water Diver courses in the previous four years) who responded to a general diving activity survey reported having diabetes (unpublished DAN AP data). Although survey data are subject to a variety of limitations including response bias, this likely indicates an increase in the number of diabetic divers in Australia.

A 2005 workshop sponsored by the UHMS and DAN led to the development of guidelines for recreational diving with diabetes mellitus. ${ }^{7}$ These guidelines describe protocols for diver selection and management of insulin during a day of diving. Although they have been largely endorsed by several diving medical organisations (including SPUMS), there are 
few data describing their uptake and utility. ${ }^{24}$ Only 16 of the 25 respondents with diabetes were aware of the UHMS/DAN or SPUMS guidelines and none reported strictly following them. Of concern is the substantially lower mean minimum pre-dive blood glucose level (BGL) of $6.1 \mathrm{mmol}^{-\mathrm{L}^{-1}}$ in this study, versus $8.3 \mathrm{mmol} . \mathrm{L}^{-1}$ recommended by UHMS/DAN, or $9.0 \mathrm{mmol} . \mathrm{L}^{-1}$ recommended by SPUMS. However, this must be interpreted cautiously. Our BGL data are derived from all respondents (including diabetics who do not use insulin) whereas the quoted recommendations apply to insulin-dependent diabetics. In addition, less than half of the respondents had consulted a diving doctor and fewer had periodic diving medical reviews. These findings identify an educational opportunity for improving practice but it is not obvious how diabetic divers or prospective divers can be encouraged to engage with a diving doctor. At the very least, we recommend that those who do so should be provided with the SPUMS or DAN/UHMS guidelines and strongly urged to follow the practice recommendations contained therein.

\section{SEPTAL DEFECTS}

There are few available data on diving with most cardiacrelated conditions. One exception to this is PFO which has received considerable attention and has been identified as a risk factor for cutaneous, inner ear, cerebral and spinal DCI. ${ }^{25-28}$ Twenty-five of the 27 respondents who reported having DCI were diagnosed with an intra-cardiac right-toleft shunt, predominantly a PFO. This was the only group with a higher proportion of females, although there appears to be no evidence of a higher incidence of PFO in women. ${ }^{29}$ Overall, this group was relatively young and did more decompression and technical diving. It is plausible that these diving activities result in higher venous gas emboli loads, therefore making the diving-related clinical complications of a septal defect more likely, which in turn makes it more likely that the defect will be detected.

Subsequent risk management through surgical intervention and/or changes to diving practice (e.g., our respondents reported less repetitive diving than other cohorts) appear to have been very successful. Similar results were reported in a study of UK divers. ${ }^{30}$ Twenty-two of the 28 respondents with a PFO in that study had been diagnosed after an episode of DCI; 20 divers had a surgical closure and 16 returned to diving. Those who continued to dive without closure successfully adopted more conservative diving practices similar to those used by the Australian cohort.

\section{CARDIAC CONDITIONS AND HYPERTENSION}

Factors such as central fluid shifts caused by immersion, exercise, cold-induced vasoconstriction, changes in gas density, static lung loads and psychological stress can increase cardiac preload, afterload, heart rate and myocardial work. Based on first principles, this would make a myocardial event more likely in predisposed individuals.
A study of 947 recreational scuba diving fatalities indicated that a myocardial event was the likely disabling injury in $26 \%$ of the deaths. ${ }^{31}$ Not surprisingly, fatality reports suggest that risk increases in older divers and in males. ${ }^{32,33}$ UK fatality data show an increasing incidence of cardiacrelated incidents against a background of an ageing diving population. ${ }^{34,35}$

Recent data suggest that $22 \%$ of Australians over 18 years old had one or more cardiovascular diseases with the prevalence being greatest in older age groups. ${ }^{36}$ It is inevitable that individuals with various degrees of cardiovascular disease are diving. A survey of Australian divers in 2000 suggested that this applied to $5.7 \%$ of respondents. ${ }^{10}$ More recent DAN AP survey data indicate that this may have been an underestimation or has subsequently increased, suggesting a prevalence of $9 \%$ in recently certified divers and 12-17\% in predominantly older, long-time and experienced divers (unpublished DAN data).

It is clear that cardiac disease is prevalent and represents a very real risk in diving and snorkelling, ${ }^{31}$ especially if it is poorly-managed. On the basis of witness reports and evidence of cardiac disease or abnormality at autopsy, many diving deaths have been attributed to arrhythmias. ${ }^{37-44}$ However, in the absence of a definitive post-mortem test this remains speculative. These Australian data also reveal that, although some victims were under treatment for cardiac disease at the time of their demise, in many their condition was undiagnosed. Any condition that increases the risk of arrhythmia and/or myocardial ischaemia may compromise safety and needs to be thoroughly investigated, preferably with involvement of a diving medical specialist. In this study, only 21 of 51 divers with ischaemic heart disease or arrhythmia reported having consulted a diving physician. A UK study also reported a low rate of diving medical consultation in those with cardiac disorders. ${ }^{30}$

\section{Arrhythmias}

A consensus discussion on cardiac disease during the 2010 DAN Fatality Workshop included a recommendation for automatic exclusion from diving for those with "arrhythmias causing impairment of exercise tolerance or consciousness". ${ }^{45}$ Fitness-to-dive considerations for an individual with arrhythmias should include factors such as the nature of the arrhythmia, the frequency and effect of episodes, the presence and type of causative disease, the treatment used and its success. In addition to considering the likelihood of an episode while diving, it is important to consider the potential adverse effects of medications, including the potential for reduced exercise capacity associated with beta-blockers or the potential for increased bleeding associated with anticoagulants.

Two of this survey's respondents with arrhythmias (20) reported at least one episode while diving, fortunately 
without injury. Most were well-controlled by medication and under diving medical oversight, but about two-thirds reported that they had not modified their diving practices (e.g., by avoiding exertion, cold water, mandatory decompression) in response to their condition.

\section{Ischaemic heart disease}

In this survey, 19 of the 31 respondents with IHD had undergone revascularisation although the degree of success is unknown. Complete revascularisation with restored exercise capacity without ischaemia may enable relatively safe diving in low-stress environments. ${ }^{46}$ However, only 10 of the 31 divers reported changing their diving behaviour. The divers with IHD were experienced, long-time divers and this might explain a reluctance to change established patterns. Only two of the 10 divers who reported having had angina had sought specific diving medical advice or changed their diving practices.

\section{Hypertension}

Given that hypertension was the most frequently managed problem in Australian general practice during $2013-14,{ }^{47}$ it is not surprising that respondents with hypertension represented the largest subgroup in this study. The majority (93 of 127 , $73 \%$ ) of hypertensive respondents were on a blood pressure control regimen. This contrasts with a UK study in which fewer $(50 \%)$ of the divers with the diagnosis of hypertension took medications, the most common being ACE inhibitors and angiotensin-II antagonists. ${ }^{30}$ None of the hypertensive divers in this study reported problems with diving over a total of 32,840 dives. Similarly, despite potential adverse effects of some medications used to manage hypertension, ${ }^{48}$ none were reported. The most common antihypertensives used were angiotensin II receptor antagonists, which have been suggested as the preferred agents for the treatment of hypertension in divers. ${ }^{48}$

\section{Medical screening}

Current diving medical screening guidelines recommend a cardiovascular review for all males at age 45 and females at age 55 years. ${ }^{45}$ Active or prospective divers should follow this advice; in any case, consult a diving medical physician in the event that they suffer from any significant chronic or acute medical condition that could be impacted by diving. Overall, only about half of this group had consulted a diving physician about their medical condition. Presumably many of the remainder may have discussed their circumstances with their general practitioner or specialist. However, in the experience of these authors, in the absence of specific diving medical knowledge, it is likely that some of the potential triggers of the diving environment may not have been sufficiently considered.

\section{LIMITATIONS}

This study has several limitations:

- The response rate of $32 \%$ may have introduced a response bias.

- The results are based on a survivor group of divers and fail to account for diving fatality victims with similar conditions that might have contributed to their demise, as well as divers who may have survived an event and stopped diving as a consequence. Therefore, the study sample may be biased towards milder forms of these conditions.

- DAN AP members are probably not typical of the general diving population. They are likely to be older, have an increased likelihood of co-existing disease, have more available funds, may travel more and may better understand their potential vulnerability and the need for and benefits of having appropriate insurance.

- Some applicants for DAN membership may have been reluctant to declare medical conditions for fear of it affecting their ability to obtain or retain insurance coverage, although it is made clear that failure to declare a relevant condition may nullify coverage. As a result, there were likely to have been more than the 833 members invited to join the MCS who were suffering from the targeted health conditions. In addition, although the survey was anonymous, it is possible that there may have been divers reluctant to disclose diving incidents owing to concern that their insurance cover might be affected. If so, this is another potential source of bias.

- The nature of some of the more historical questions may have introduced a recall bias.

- Many responses were excluded from the analysis due to missing replies to certain questions.

- The number of dives performed by divers with particular conditions is relatively small for the purposes of an epidemiological evaluation. Our ability to interpret the associated risks is therefore limited.

\section{Conclusions}

These results are limited by the $32 \%$ response rate and potential for bias towards selection of those most careful with their health. However, a substantial minority of DAN AP members are diving with medical conditions such as cardiac conditions, hypertension, diabetes and asthma which could potentially impact the safety of their diving. Many of the conditions were controlled by medications, several of which could themselves have adverse effects while diving. Surgical intervention was common for divers diagnosed with a PFO or ischaemic heart disease. Only one quarter of the divers reported modifying their diving practices to accommodate their condition, and only half had sought specialist diving medical advice. Despite the obvious concerns, our respondents with known and largely managed medical problems have dived with very few 
incidents associated with their conditions. Conclusions based on survivor populations must be cautious and our numbers remain relatively small, but it does seem that, if these medical conditions are identified and managed appropriately, the risks associated with diving may be acceptable. More research with larger cohorts is needed to better understand the risks in an ageing comorbid diving population.

\section{References}

1 Vorosmarti J, Linaweaver Jr PG, editors. Fitness to dive. 34th Undersea and Hyperbaric Medical Society Workshop. UHMS Publication Number 70(WS-WD)5-1-87. Bethesda: Undersea and Hyperbaric Medical Society; 1987.

2 Davies D. SPUMS statement on diabetes. SPUMS Journal. 1992;22:31-2.

3 Victorian Asthma Foundation. Policy of the Victorian Asthma Foundation. SPUMS Journal. 1987;17:133-4.

4 Lippmann J, Taylor D McD, Stevenson C, Mitchell S. The demographics and diving behaviour of DAN Asia-Pacific members with and without pre-existing medical conditions. Diving Hyperb Med. 2016;46:200-6.

5 National Statistical Service. Sample size calculator. [cited 2016 December 09]. http://www.nss.gov.au/nss/home.NSF/ pages/Sample+size+calculator.

6 South Pacific Underwater Medicine Society. Guidelines for medical risk assessment for recreational diving. Appendix B: suggested assessment for the diver with diabetes, 2011. [cited 2017 June 25]. Available from: https://www.spums.org.au/ content/spums-full-medical-0. (restricted access).

7 Mitchell SJ, Taylor L, Bennett MH. Diabetes in recreational diving: a protocol for the future. In: Pollock NW, Uguccioni DM, Dear G deL, editors. Diabetes and recreational diving guidelines for the future. Proceedings of the Undersea and Hyperbaric Medical Society/Divers Alert Network 2005 Workshop, Las Vegas, NV, USA. Durham NC, Divers Alert Network; 2005. p 86-100.

8 Farrell PJS. Asthmatic amateur divers in the UK. SPUMS Journal. 1995;25:22.

9 Hanson E, Fleisher J, Jackman RP, Uguccioni DM, Thalmann ED, Cadahy EA. Demographics and illness prevalence in a recreational scuba diver population: fitness to dive [abstract]. Undersea Hyperb Med. 1999;26(Supp):48.

10 Taylor DM, O'Toole KS, Ryan CM. Experienced, recreational scuba divers in Australia continue to dive despite medical contra-indications. Wilderness Environ Med. 2002;13:187-93.

11 Tetzlaff K, Muth CM. Demographics and respiratory illness prevalence of sport scuba divers. Int J Sports Med. 2005;26:607-10.

12 Beckett A, Kordfick MF. Risk factors for dive injury. Res Sports Med. 2007;15:201-11.

13 Corson K, Moon RE, Nealen ML, Dovenbarger JA, Bennett PB. A survey of diving asthmatics [abstract]. Undersea Biomed Res. 1992;19S:18-9.

14 United Kingdom Diving Medical Committee (UKDMC). Asthma. [cited 2016 December 09]. Available from: http:// ukdmc.org/asthma.

15 Glanvill P, St Leger Dowse M, Bryson P. A longitudinal cohort study of UK divers with asthma: diving habits and asthma health issues. SPUMS Journal. 2005;35:18-22.

16 Walker D. Report on Australian diving deaths 1972-1993. Melbourne: JL Publications; 1998.
17 Davis M, Warner M, Ward B. Snorkelling and scuba diving deaths in New Zealand, 1980-2000. SPUMS Journal. 2002;32:70-80.

18 Bennett M. The SPUMS recreational diving medical. Appendix C: suggested assessment for the diver with asthma. Diving Hyperb Med. 2010;40:151-3.

19 Edge CJ, Grieve AP, Gibbons N, O'Sullivan F, Bryson P. Control of blood glucose in a group of diabetic scuba divers. Undersea Hyperb Med. 1997;24:201-7.

20 Dear GdL, Pollock NW, Uguccioni DM, Dovenbarger $\mathrm{J}$, Feinglos MN, Moon RE. Plasma glucose responses in recreational divers with insulin-requiring diabetes. Undersea Hyperb Med. 2004;31:291-301.

21 Edge CJ, St Leger Dowse M, Bryson P. Scuba diving with diabetes mellitus - the UK experience 1991-2001. Undersea Hyperb Med. 2005;32:27-37.

22 Pollock NW, Uguccioni DM, Dear GdL, Bates S, Albushies TM, Prosterman SA. Plasma glucose response to recreational diving in novice teenage divers with insulin-requiring diabetes mellitus. Undersea Hyperb Med. 2006;33:125-33.

23 Pieri M, Cialoni D, Marroni A. Continuous real-time monitoring and recording of glycemia during scuba diving: pilot study. Undersea Hyperb Med. 2016;43:265-72.

24 Johnson R. A day in the life of a diabetic diver: the Undersea and Hyperbaric Medical Society/Divers Alert Network protocol for diving with diabetes in action. Diving Hyperb Med. 2016;46:181-5.

25 Moon RE, Camporesi EM, Kisslo JA. Patent foramen ovale and decompression sickness in divers. Lancet. 1989;11:513-4.

26 Wilmshurst PT, Byrne JC, Webb-Peploe MM. Relation between interatrial shunts and decompression sickness in divers. Lancet. 1989;2:1302-6.

27 Wilmshurst PT, Morrison WL, Walsh KP. Comparison of the size of persistent foramen ovale and atrial septal defects in divers with shunt-related decompression illness and in the general population. Diving Hyperb Med. 2015;45:89-93.

28 Wilmshurst PT. The role of persistent foramen ovale and other shunts in decompression illness. Diving Hyperb Med. 2015;45:98-104.

29 Fisher DC, Fisher EA, Budd JH, Rosen SE, Goldman ME. The incidence of patemt foramen ovale in 1,000 consecutive patients. A contrast transoesophageal echocardiography study. Chest. 1995;107:1504-9.

30 St Leger Dowse M, Waterman MK, Penny CE, Smerdon GR. Does self-certification reflect the cardiac health of UK sport divers? Diving Hyperb Med. 2015;45:184-9

31 Denoble PJ, Caruso JL, de L Dear G, Vann RD. Common causes of open-circuit recreational diving fatalities. Undersea Hyperb Med. 2008;35:393-406.

32 Lippmann J, Baddeley A, Vann R, Walker D. An analysis of the causes of compressed gas diving fatalities in Australia from 1972-2005. Undersea Hyperb Med. 2013;40:49-61.

33 Denoble PJ, Pollock NW, Vaithiyanathan P, Caruso JL, Dovenbarger JA, Vann RD. Scuba injury death rate among insured DAN members. Diving Hyperb Med. 2008;38:182-8.

34 Cumming B, Peddie C, Watson. A review of the nature of diving in the United Kingdom and of diving fatalities (1998 2009). In: Vann RD, Lang MA, editors. Recreational diving fatalities. Proceedings of the Divers Alert Network 2010 April 8-10 Workshop. Durham, NC: Divers Alert Network; 2011. p. 99-117.

35 Cumming B. National Diving Committee (NDC) diving incidents report 2015. Elmsmere Port, Cheshire: British Sub- 
Aqua Club. [cited 2017 June 26]. Available from: http://www. bsac.com/page.asp?section $=1038 \&$ sectionTitle $=$ Annual+Div ing+Incident+Report.

36 Australian Institute of Health and Welfare (AIHW). How many Australian have cardiovascular disease. 2011-2012. [cited 2016 Dec 13]. Available from: http://www.aihw.gov. $\mathrm{au} /$ cardiovascular-disease/prevalence.

37 Lippmann J, Lawrence C, Fock A, Jamieson S, Harris R. Provisional report on diving-related fatalities in Australian waters 2011. Diving Hyperb Med. 2016; 46:207-40.

38 Lippmann J, Lawrence C, Fock A, Jamieson S. Provisional report on diving-related fatalities in Australian waters 2010. Diving Hyperb Med. 2015;44:154-175.

39 Lippmann J, Lawrence C, Wodak T, Fock A, Jamieson S. Provisional report on diving-related fatalities in Australian waters 2009. Diving Hyperb Med. 2013;43:194-217.

40 Lippmann J, Lawrence C, Wodak T, Fock A, Jamieson S, Walker D, Harris R. Provisional report on diving-related fatalities in Australian waters 2008. Diving Hyperb Med. 2013;43:16-34.

41 Lippmann J, Walker D, Lawrence C, Fock A, Wodak T, Jamieson S. Provisional report on diving-related fatalities in Australian waters 2007. Diving Hyperb Med. 2012;42:151170.

42 Lippmann J, Walker D, Lawrence C, Fock A, Wodak T, Jamieson S. Provisional report on diving-related fatalities in Australian waters 2006. Diving Hyperb Med. 2011;41:70-84.

43 Walker D, Lippmann J, Lawrence C, Fock A, Wodak T, Jamieson S. Provisional report on diving-related fatalities in Australian waters 2005. Diving Hyperb Med. 2010;40:131-49.

44 Walker D, Lippmann J, Lawrence C, Houston J, Fock A. Provisional report on diving-related fatalities in Australian waters 2004. Diving Hyperb Med. 2009;39:138-61.

45 Mitchell SJ, Bove AA. Medical screening of recreational divers for cardiovascular disease: consensus discussion at the
Divers Alert Network Fatality Workshop. Undersea Hyperb Med. 2011;38:289-96.

46 Bove AA. The cardiovascular system and diving risk. Undersea Hyperb Med. 2011;38:261-9.

47 Britt H, Miller GC, Henderson J, Bayram C, Harrison C, Valenti L, et al. General practice activity in Australia 2013-14. General practice series no. 36. Sydney: Sydney University Press, 2013. [cited 2016 Dec 14]. Available from: https://ses. library.usyd.edu.au/bitstream/2123/11882/4/9781743324226_ ONLINE.pdf.

48 Bennett M. Drugs and diving. In: Edmonds C, Bennett M, Lippmann J, Mitchell S, editors. Diving and subaquatic medicine, 5th ed. Boca Raton, FL: Taylor and Francis; 2016. p. 497-507.

\section{Acknowledgements}

The authors wish to acknowledge Scott Jamieson for his assistance with data collection and Michael Lippmann for his assistance in the construction of the survey platform.

\section{Conflicts of interest}

John Lippmann is the Founder and Chairman of DAN AP. DAN is involved in the collection and reporting of dive accident data and provides evacuation cover and dive injury insurance to recreational divers.

\section{Funding}

This study was funded by DAN AP.

Submitted: 18 April 2017; revised 20 and 28 July 2017 Accepted: 02 August 2017

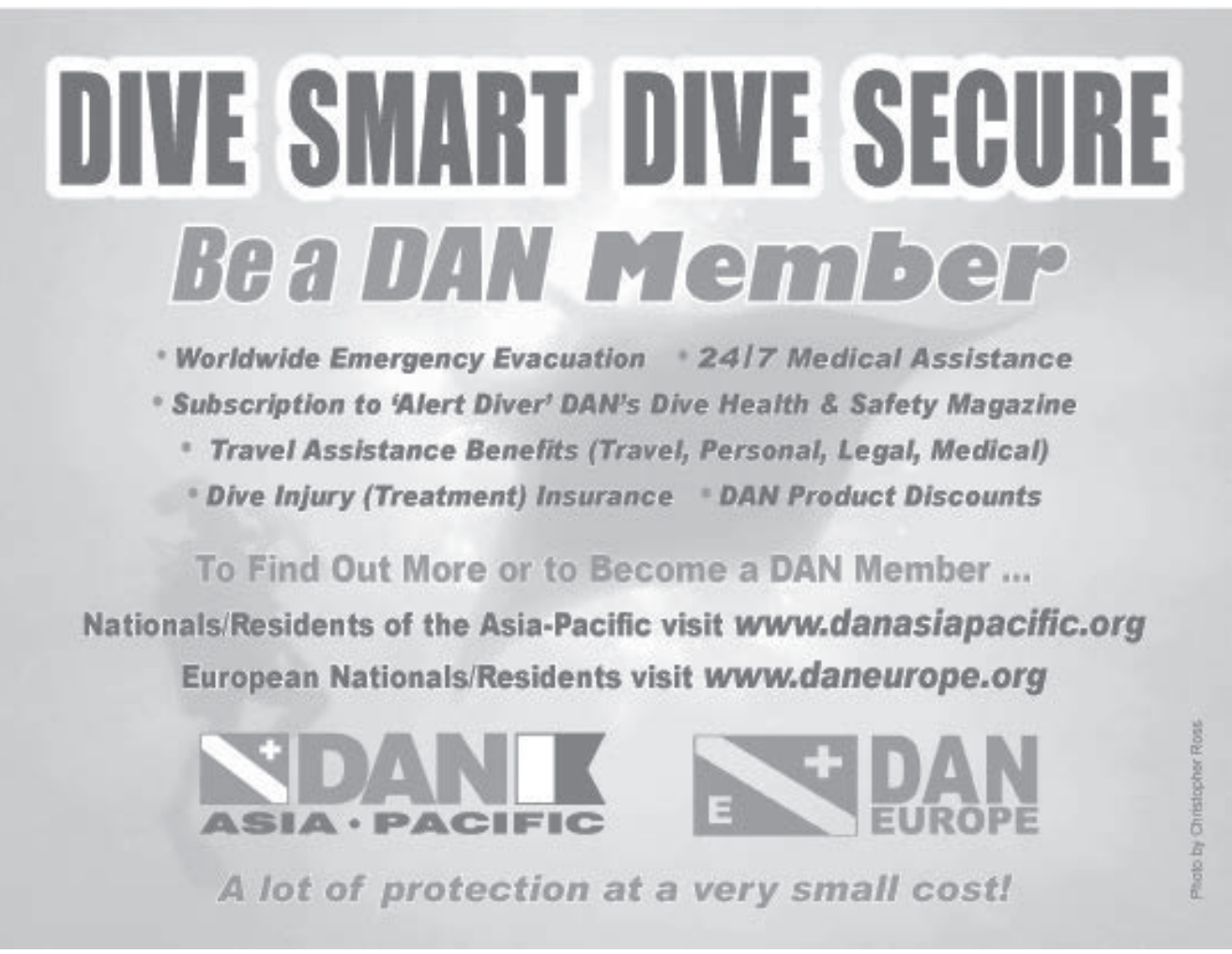




\title{
Technical report
}

\section{A pleural vacuum relief device for pleural drain unit use in the hyperbaric environment}

\author{
Marco Gelsomino, Theo Tsouras, lan Millar, Andrew Fock \\ Hyperbaric Medicine Service, The Alfred Hospital, Melbourne, Victoria, Australia \\ Corresponding author: Marco Gelsomino, Kleinhüningerstrasse 177, 4057 Basel, Switzerland \\ marco.gelsomino@gmail.com
}

\section{Key words}

Equipment; Chest tubes; Suction; Patient monitoring; Lung; Barotrauma; Pressure

\begin{abstract}
(Gelsomino M, Tsouras T, Millar I, Fock A. A pleural vacuum relief device for pleural drain unit use in the hyperbaric environment. Diving and Hyperbaric Medicine. 2017 September;47(3):191-197.)

Introduction: When a standard water-seal pleural drain unit (PDU) is used under hyperbaric conditions there are scenarios where excessive negative intrapleural pressure (IPP) and/or fluid reflux can be induced, risking significant morbidity. We developed and tested a pleural vacuum relief (PVR) device which automatically manages these risks, whilst allowing more rapid hyperbaric pressure change rates.

Methods: The custom-made PVR device consists of a one-way pressure relief valve connected in line with a sterile micro filter selected for its specific flow capacity. The PVR device is designed for connection to the patient side sampling port of a PDU system, allowing inflow of ambient air whenever negative pressure is present, creating a small, controlled air leak which prevents excessive negative pressure. The hyperbaric performance of a Pleur-Evac A-6000 intercostal drain was assessed with and without this added device by measuring simulated IPP with an electronic pressure monitor connected at the patient end of the PDU. IPP readings were taken at 10, 15, 20 and $30 \mathrm{cmH}_{2} \mathrm{O}$ of suction (set on the drain unit) at compression rates of $10,30,60,80,90$ and $180 \mathrm{kPa} \cdot \mathrm{min}^{-1}$ to a pressure of $280 \mathrm{kPa}$.

Results: At any compression rate of $>10 \mathrm{kPa} \cdot \mathrm{min}^{-1}$, the negative IPP generated by the Pleur-Evac A-6000 alone was excessive and resulted in back flow through the PDU water seal. By adding the PVR device, the generated negative IPP remains within a clinically acceptable range, allowing compression rates of at least $30 \mathrm{kPa} \cdot \mathrm{min}^{-1}$ with suction settings up to $-20 \mathrm{cmH}_{2} \mathrm{O}$ during all phases of hyperbaric treatment.

Conclusions: The PDU PVR device we have developed works well, minimising attendant workload and automatically avoiding the excessive negative IPPs that can otherwise occur. This device should only be used with suction.
\end{abstract}

\section{Introduction}

Proprietary water-seal pleural drain units (PDUs) are the preferred method for chest drainage of pneumothorax, empyema, blood or fluid during in-hospital care. On occasions, patients with an intercostal catheter (ICC) and a PDU will require hyperbaric oxygen treatment (HBOT) to manage a coexistent condition, such as decompression illness (DCI), cerebral arterial gas embolism (CAGE) or necrotizing fasciitis. The designs of PDUs incorporate multiple air-filled spaces on either side of a water trap and these are affected by hyperbaric exposure in ways that depend upon whether the unit is on suction or not, and whether the patient has a pleural air leak or not. A key performance requirement for pleural drain systems is the avoidance of excessively negative intra-pleural pressure (IPP) as this may result in lung injury and potentially worsening of a trans-pulmonary air leak. ${ }^{1}$ Although these units have manual and automatic pressure relief systems designed to prevent excess negative pressure, the automatic relief settings are generally quite high. Further, some hyperbaric scenarios result in a bubbling backflow of water-seal fluid towards the patient, for example during fast compression for CAGE, and this risks contamination of what should be the sterile side of the system. To safely manage a patient with a PDU during HBOT, close observation is necessary and a number of modifications must be made to standard pleural drainage system care. To avoid potential complications arising from use of a proprietary PDU, many hyperbaric units and hyperbaric texts recommend that a PDU be disconnected with the substitution of a Heimlich valve during HBOT.

Previous studies have shown that PDUs from different manufacturers show variations from the prescribed to the actual delivered intra-pleural pressure (IPP)., ${ }^{2,3}$ Our group has previously tested the function of one PDU (Atrium Oasis Dry Suction 3600 Chest Drain) under hyperbaric conditions; this device proved to be dramatically affected by pressure changes. ${ }^{4}$ However, safe use in an hyperbaric environment was possible if no suction was applied during pressurisation 
Figure 1

A schematic of the Pleur-Vac A-6000 pleural drainage unit (PDU) and of the experimental set up including the pleural vacuum relief(PVR) device

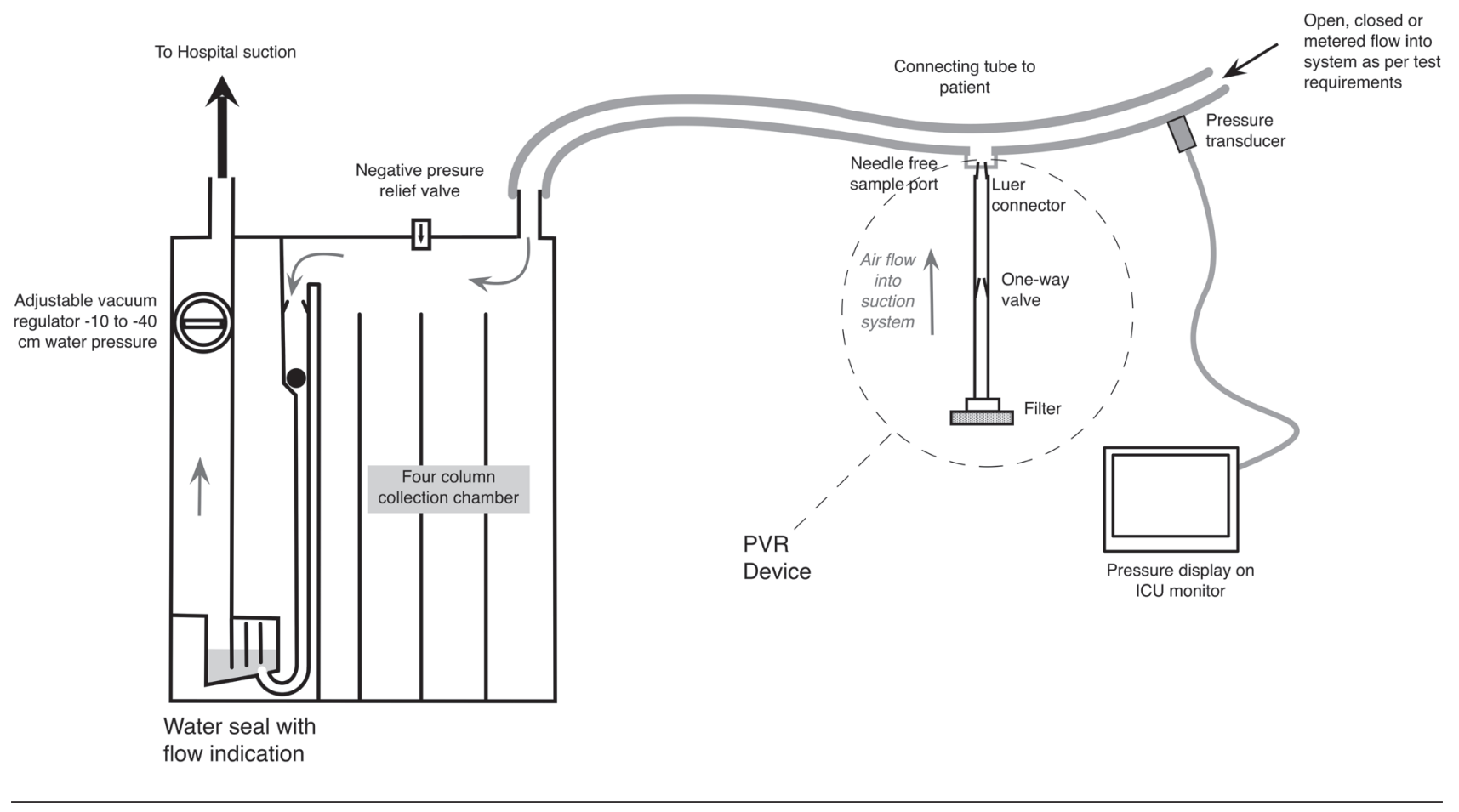

and if the rate of pressurisation was limited to $10 \mathrm{kPa} \cdot \mathrm{min}^{-1}$ or less. Under these conditions the water-seal was maintained and the column of water making the seal did not back flow into the collection chamber. ${ }^{5}$

As the construction details of different PDUs vary significantly, the performance characteristics of any one particular model cannot be assumed to be the same as for other similar devices. ${ }^{4}$ Currently our institution predominantly uses the Pleur-Evac ${ }^{\circledR}$ A-6000 PDU from Teleflex. In this device the degree of suction is controlled by an inbuilt regulator with the desired suction selected via a crude rotary dial. A water seal chamber indicates the negative pressure delivered on the patient side of the device, with bubbles through the water seal chamber indicating the rate of any air leakage present. The PDU collection chamber is a closed volume, so that when the ambient pressure increases, vacuum/negative pressure will develop in the collection chamber unless this is relieved by inflow of gas or fluid. In the absence of an air leak from the patient, the only inflow route for this gas is via back flow through the water seal (Figure 1). While for many patients removal of suction during pressurisation may be clinically acceptable, it is usually considered optimal to maintain stability of the IPP at all times by maintaining the suction to the PDU during all phases of HBOT.

The strategy recommended by the manufacturer for avoiding excess negative pressure is to manually operate the negative pressure relief valve located on top of the unit. Although this does work during pressurisation, there are several disadvantages: the patient attendant becomes occupied physically and mentally with keeping the PDU vented, to the detriment of attending to other matters, and negative IPP pressure can disappear when the valve is open for prolonged periods, risking lung collapse. These problems are multiplied when more than one PDU is present.

Our clinical experience with patients with pleural air leaks and PDUs on suction suggested that keeping the PDU on regulated suction with an artificially provided and controlled 'air leak' might mitigate the potential problems associated with PDUs in hyperbaric situations. This would enable continuance of suction and the desired low level negative IPP pressure in patients without pleural leaks throughout the various phases of hyperbaric treatment. After a prototype device appeared to work in crude testing, we refined the concept, and undertook detailed testing of the new device by comparing the performance of the Pleur-Evac A-6000 with and without the pleural vacuum relief device across a range of pleural drainage and hyperbaric settings.

\section{Aims}

We hypothesised that a custom-made pleural vacuum relief (PVR) device inserted into the patient tube side of a PDU via its needleless sample site (an auto-sealing 'Luer-Lock' style push-and-twist connection) would allow passive, controlled introduction of ambient air into the PDU system whilst the PDU was on suction, ensuring that the desired low levels of negative pressure would not become excessive, thus allowing for intervention-free operation of the ICC 
Figure 2

The custom-made pleural vacuum relief (PVR) device will be inserted into the patient tube side of a pleural drainage unit (PDU) via the PDU's needleless sample site

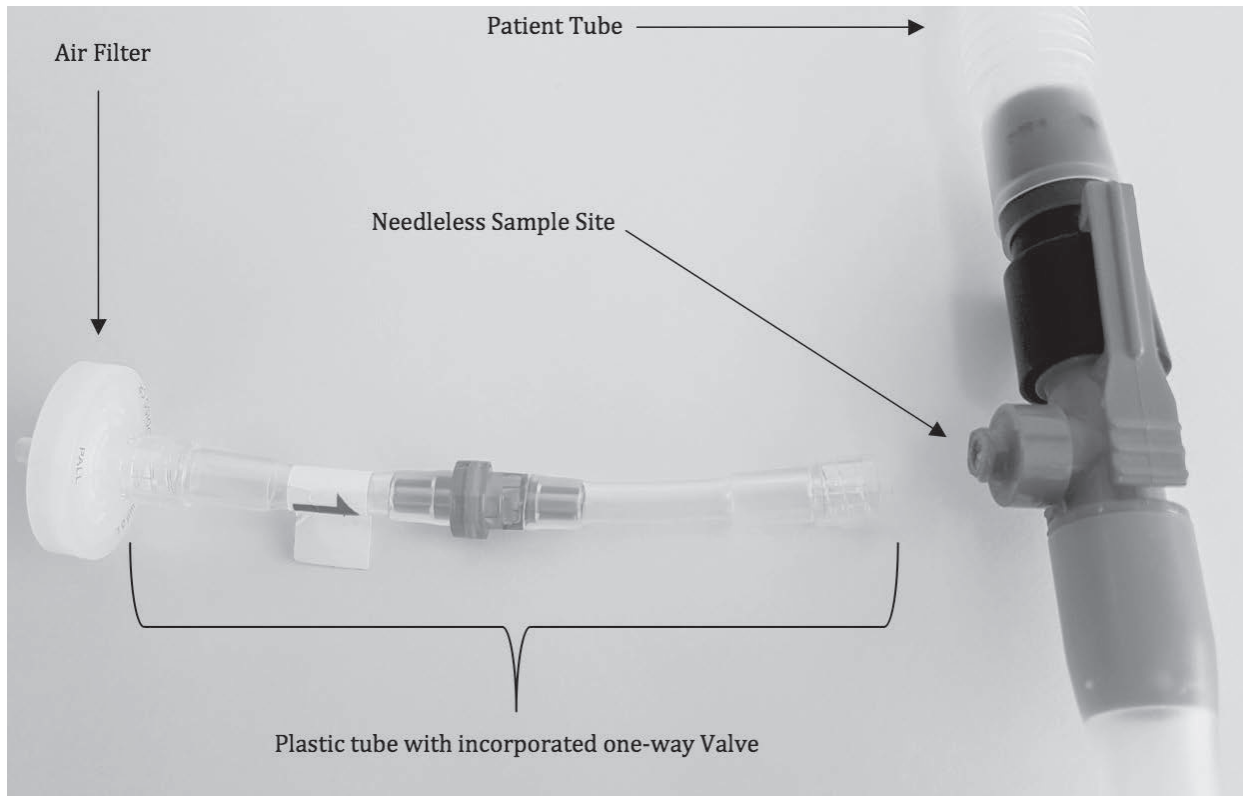

and PDU during all phases of HBOT. We tested the ability of the PVR device to allow the PDU to remain on suction and maintain a safe IPP throughout hyperbaric exposure during both normal and elevated pressurisation rates. ${ }^{5}$ As the testing process involved use of the Pleur-Evac A-6000 PDU with and without the PVR device, we are also able to report details of the hyperbaric performance of the PleurEvac A-6000 PDU.

\section{Methods}

This study did not involve human or animal subjects and, therefore, was not subject to ethics committee approval or application. Testing was performed on a single Pleur-Evac A-6000 PDU device of the type currently used in The Alfred Hospital Department of Intensive Care and Hyperbaric Medicine, Melbourne. The drain tube normally connected to the patient was connected to an electronic pressure monitor (Edwards Life sciences, Pressure Monitoring Kit with TruWave Disposable Pressure Transducer, Ref: PX212) to measure the generated pressure at the patient end of the drain tube. It was assumed that this would equate to the IPP in a patient without a pleural leak. The pressure transducer accuracy was verified with a calibrated test instrument (Druck® DPI 705 Digital Pressure Indicator, Rep: 107953, s/n: 70526859, calibrated: 25/10/2012). Pressure readings were taken by connecting the pressure transducer via a chamber penetration to a Phillips IntelliVue MP70 monitor external to the chamber, so that no staff were required to be pressurised in the hyperbaric chamber during the study. Pressure readings were read from the monitor visually and transcribed onto an Excel spreadsheet (Microsoft@ 2004). A schema of the experimental set up is presented in Figure 1.
The PDU was set up as per the manufacturer's instructions with the water seal chamber filled to the 'fill line'. The PDU suction port was connected via standard medical suction tubing to the hyperbaric chamber wall suction outlet set to maximum (The Alfred Hyperbaric Service chamber is a TGA-approved chamber and, as such, all gas and suction supplies conform to the appropriate Australian gas standard AS 2896-2011). In this configuration, the PDU suction level is controlled by the suction regulator incorporated into the PDU as per manufacturer recommendations.

The PVR device (Figure 2) allows a small flow of ambient air to be entrained throughout the various phases of a typical HBOT treatment whenever the PDU is on suction, unless the patient were to have a very large pleural air leak. The flow of air through the device is limited by the flow resistance properties of the device such that with the suction connected, sufficient continuous flow is maintained through the PDU to prevent the development of excessive negative pressure within the PDU collecting chamber while still maintaining the desired negative ICC pressure within clinically acceptable values. With continuous bubbling occurring through the water trap, it becomes easy to verify that the system is working satisfactorily.

The PVR device consists of a non-return valve and a small microbiological filter in line. Selecting the optimal filter involved bench testing over a dozen types of filters using the NATA-certified PTS2000 flow bench tester to determine pressure drop characteristics across each filter. The PALL Life Sciences Acrodisc $®$ CR $25 \mathrm{~mm}$ syringe filter with $1 \mu \mathrm{m}$ PTFE membrane, (PN S4226, Lot 21683800) was selected as this provided the appropriate properties when incorporated 
into our PVR device. ${ }^{6,7}$ The controlled leak results in only a small, inconsequential reduction in the negative pressure at the ICC end of the patient tube during steady-state pressure conditions. Should the patient cough, back flow through the PVR is prevented by the presence of a one-way valve. The disposable device is easy to assemble and connect to the PDU. During compression, a whistling noise is commonly heard coming from the device, which gives a supplementary auditory confirmation that the PVR valve is working.

Having refined the PVR device, we undertook testing with a pressure-monitored PDU during various suction and hyperbaric pressure scenarios in the hyperbaric chamber (FETL-24, Fink Engineering, Melbourne, Australia). The suction conditions examined were:

- $\quad$ suction tubing connected to the chamber suction, with suction switched off ('no suction');

- $\quad$ suction tubing connected to the chamber suction, with suction switched on, regulated by the PDU's adjustable suction regulator to $10,15,20$ and $30 \mathrm{cmH}_{2} \mathrm{O}$ of suction $\left(30 \mathrm{cmH}_{2} \mathrm{O}\right.$ was only tested at $30 \mathrm{kPa} \cdot \mathrm{min}^{-1}$ pressurisation rate).

The hyperbaric chamber rates of pressure change used were:

- compression at $10,30,60,80,90$ and $180 \mathrm{kPa} \cdot \mathrm{min}^{-1}$ to $280 \mathrm{kPa}$;

- decompression was conducted at $60 \mathrm{kPa} \cdot \mathrm{min}^{-1}$ for all tests.

The nominated compression rate for all Royal Navy/US Navy treatment tables (until the latest iteration of the USN dive manual) has been $180 \mathrm{kPa} \cdot \mathrm{min}^{-1}$. Anecdotally, most clinical hyperbaric services pressurize at slower rates than this with many commercial and civilian chambers not physically capable of this compression rate. At the Alfred the routine compression rates are:

- $\quad$ Standard treatment table (TT) $10 \mathrm{kPa} \cdot \mathrm{min}^{-1}$;

- $\quad$ ICU TT $30 \mathrm{kPa} \cdot \mathrm{min}^{-1}$;

- Diver emergency TT $180 \mathrm{kPa} \cdot \mathrm{min}^{-1}$.

The level of negative pressure within the ICC connection (simulated IPP) was recorded every $5,15,30$ or 60 seconds depending upon the pressurisation rate being studied.

For the $30 \mathrm{kPa} \cdot \mathrm{min}^{-1}$ pressurisation rate, we also conducted measurements with an ICC tubing air leak to simulate a bronchopleural fistula. Fistula flows of 1, 2.5 and $5 \mathrm{~L} \cdot \mathrm{min}^{-1}$ were simulated. This was conducted by using a chamber oxygen outlet connected via a low-flow flow meter and a Y-connector to the patient tube.

The outcome measures were:

- $\quad$ simulated intra-pleural pressure (IPP);

- displacement distance of the underwater seal column towards the ball valve.
An initial 'proof of concept' series of tests was performed which demonstrated a very low inter-test variance in measured values of IPP between different 'runs' of the same pressurisation profile. As such, it was decided that three 'runs' for each set of conditions would provide adequate data to calculate mean IPP values for that set of conditions. A pilot study had shown that pressure readings in other areas of the ICC system were not relevant to the delivery of the correct IPP. ${ }^{4}$

\section{Results}

\section{NO SUCTION, NO PVR DEVICE}

At a compression rate of $10 \mathrm{kPa} \cdot \mathrm{min}^{-1}$ without suction, the negative IPP rose without backflow of the underwater seal fluid into the collection chamber. In this test, the IPP increased to $-26 \mathrm{cmH}_{2} \mathrm{O}$ during the first minute (the negative pressure in the patient tube increased as increasing ambient pressure led to a decrease of air volume in the collection chamber) without breakdown of the water seal, then stabilised around $-22 \mathrm{cmH}_{2} \mathrm{O}$ until the ambient pressure reached $280 \mathrm{kPa}$. The IPP then remained stable at pressure, before coming back to zero and then becoming slightly positive during decompression. At all compression rates faster than $10 \mathrm{kPa} \cdot \mathrm{min}^{-1}$, the flow of gas backwards into the collecting chamber forces the water in the underwater seal up the column and past the ball valve into the collecting chamber potentially compromising the seal should too much fluid be lost.

\section{NO SUCTION, WITH PVR DEVICE}

At the compression rates of $10,30,60$ and $180 \mathrm{kPa} / \mathrm{min}$ with no suction applied and with the PVR device in place, the profiles of the IPP pressure curves followed a similar pattern in each test. During compression, the IPP rapidly became more negative, then stabilised while at $280 \mathrm{kPa}$. During decompression the negative IPP decreased with the decrease in pressure in the chamber, returning to baseline (zero) after completion of decompression. At each compression rate no backflow of the water column making the seal was noted. Maintenance of this volume is essential for correct functioning of the device. The maximum negative IPP pressure recorded was dependent upon the compression rate, $-9,-16,-28$ and $>-42 \mathrm{cmH}_{2} \mathrm{O}$ at compression rates of 10 , 30,60 and $180 \mathrm{kPa} \cdot \mathrm{min}^{-1}$ respectively. A negative pressure of $-42 \mathrm{cmH}_{2} \mathrm{O}$ was the maximum our chosen electronic pressure transducer could measure. It was observed that the suction delivered to the patient tube was more negative than the suction regulator setting on our ICC drain in all phases of the HBOT cycle.

\section{WITH SUCTION, WITH PVR DEVICE}

In all suction conditions and at all compression rates tested with the PVR device in place, the water seal was preserved. At compression rates of 10 and $30 \mathrm{kPa} \cdot \mathrm{min}^{-1}$ and with 
Figure 3

Average intrapleural pressure (IPP) during all phases of the pressurisation cycle; compression rate $30 \mathrm{kPa} \cdot \mathrm{min}^{-1}$; decompression rate $60 \mathrm{kPa} \cdot \mathrm{min}^{-1}$, with and without leak

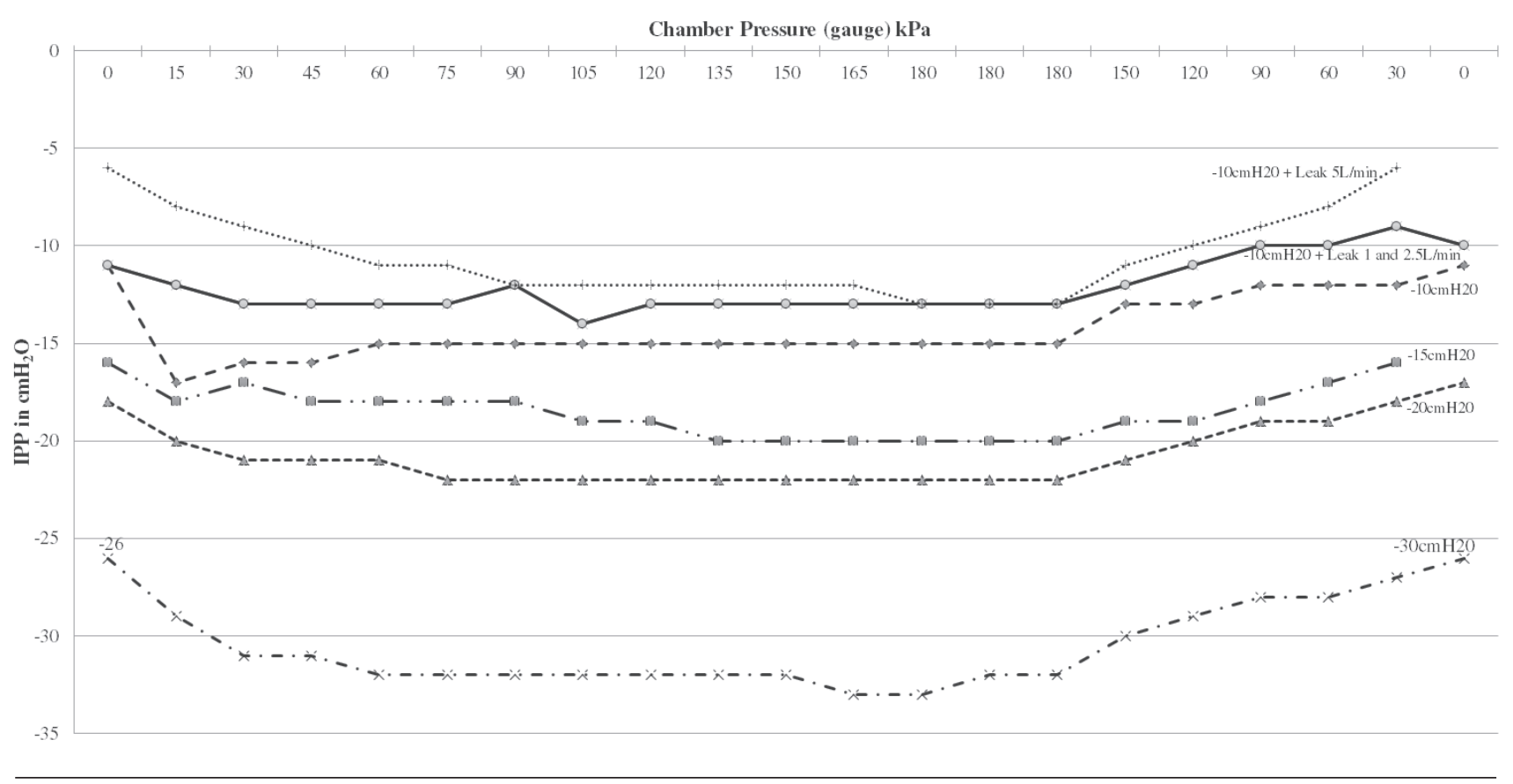

Figure 4

Average intrapleural pressure (IPP) during all phases of the pressurisation cycle; compression rate $60 \mathrm{kPa} \cdot \mathrm{min}^{-1}$; decompression rate $60 \mathrm{kPa} \cdot \mathrm{min}^{-1}$

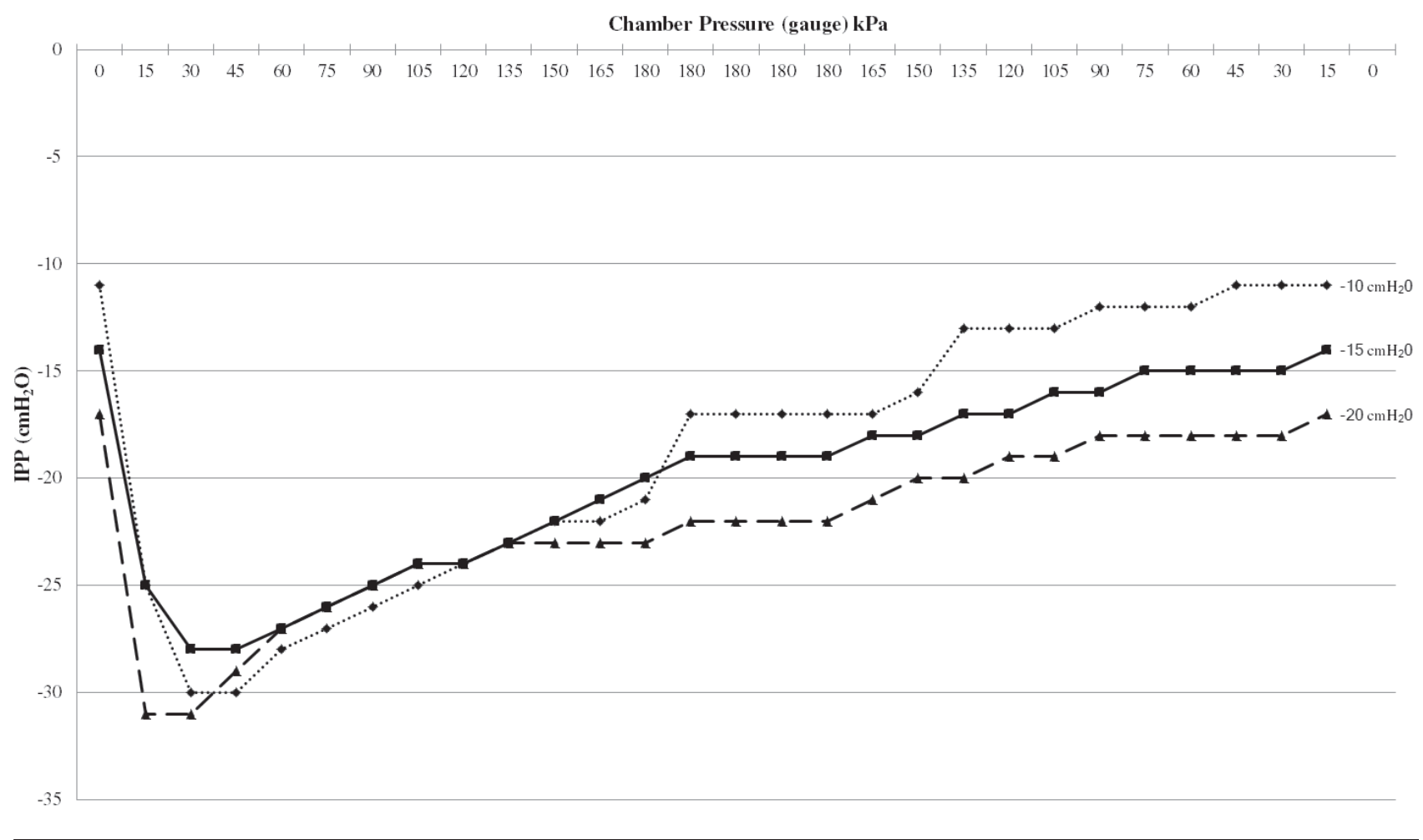

variation of the suction setting from -10 to $-20 \mathrm{cmH}_{2} \mathrm{O}$ (and $-30 \mathrm{cmH}_{2} \mathrm{O}$ at $\left.30 \mathrm{kPa} \cdot \mathrm{min}^{-1}\right)$, there was only a slight fall in the initial IPP values and in the maximum negative values. Changes during $30 \mathrm{kPa} \cdot \mathrm{min}^{-1}$ compression are shown in Figure 3. Overall, the negative IPP generated remained much closer to the regulator suction setting on the PDU. With a compression rate of $60 \mathrm{kPa} \cdot \mathrm{min}^{-1}$ a somewhat larger initial fall in IPP occurred but did not exceed $-30 \mathrm{cmH}_{2} \mathrm{O}$ and was the same for all suction settings tested (Figure 4). 
At the maximum compression rate tested of $180 \mathrm{kPa} \cdot \mathrm{min}^{-1}$ the initial pressure drop was greater and exceeded the -42 $\mathrm{cmH}_{2} \mathrm{O}$ limit of the electronic pressure monitor display for the two suction settings of -10 and $-20 \mathrm{cmH}_{2} \mathrm{O}$. The maximum compression rate at which the IPP did not exceed $-42 \mathrm{cmH}_{2} \mathrm{O}$ with a suction setting of $-10 \mathrm{cmH}_{2} \mathrm{O}$ was found to occur with a compression rate of just under $90 \mathrm{kPa} \cdot \mathrm{min}^{-1}$.

\section{WITH SUCTION, WITH PVR DEVICE, WITH AIR LEAK}

At the compression rate of $30 \mathrm{kPa} \cdot \mathrm{min}^{-1}$ with the suction set to $-10 \mathrm{cmH}_{2} \mathrm{O}$, air was allowed to enter the patient tubing in a regulated fashion to simulate a bronchopleural fistula. The test results demonstrated very stable IPP during all phases of the HBOT with the measured negative IPP falling below the set suction on the ICC drain only with a simulated pleural air leak of $51 \cdot \mathrm{min}^{-1}$. However, the IPP remained negative at around $-6 \mathrm{cmH}_{2} \mathrm{O}$ during the initial and final phases and not exceeding $-12 \mathrm{cmH}_{2} \mathrm{O}$ overall (Figure 3 ).

\section{Discussion}

It is optimal for most intensive care interventions, including thoracic drainage if present, to be continued throughout HBOT and during transfers when technically feasible rather than disconnecting and replacing them with alternative technology. Continuous intrapleural suction, particularly during decompression, is critically important in treating pneumothorax which will otherwise expand, potentially leading to a tension pneumothorax. The PDU study previously undertaken on the Atrium Oasis Dry Suction 3600 chest drain showed significant variations in the degree of negative IPP generated during compression and decompression. ${ }^{4}$ Acceptable levels of IPP were only achievable when compression rates were very slow and when suction was discontinued during compression. Given also the potential for backflow of the underwater seal fluid into the collection chamber and subsequent loss of the seal, there is a requirement for the chamber attendant to monitor the PDU water-seal during compression, a period which already involves heavy task loading, especially in highacuity patients.

We have confirmed that the Pleur-Evac A-6000 ICC drain is generally safe to use if the same procedures are used as those recommended previously, ${ }^{4}$ that is, without suction during compression and with the compression rate limited to $10 \mathrm{kPa} \cdot \mathrm{min}^{-1}$.

Our primary aim was to develop a safe, cheap and easyto-use system which would minimise the risk of excessive negative intra-pleural pressures whilst allowing normal operation of proprietary PDUs, including continuity of suction, during all phases of HBOT. This device would ideally require minimal or no intervention by staff and would allow rates of compression faster than $10 \mathrm{kPa} \cdot \mathrm{min}^{-1}$. The introduction of this PVR device has allowed the use of standard treatment tables in our institution when a PDU is present and has markedly reduced inside attendant workload in critically ill patients. Also, this device does not modify or change the intended use of the PDU in any way.

A valid criticism of this type of device is that it can allow air to flow into the pleural cavity in the absence of suction. This scenario was tested as a potential 'failure mode'. The internal resistance within the PVR device will prevent rapid re-accumulation of a pneumothorax in the event of suction being disconnected before the PVR has been removed but it is imperative that staff are educated about safe use of the device and that there is strict adherence to protocols and checklists. We require removal and disposal of each PVR at the conclusion of each hyperbaric session and prior to transport back to the ICU.

Even with the use of our PVR device, the negative IPP generated during hyperbaric compression was always higher than the set suction on the suction regulator setting of the ICC drain. Normal intrapleural pressures vary between -8 to $-3.4 \mathrm{cmH}_{2} \mathrm{O}$ but can transiently exceed -54 and +70 $\mathrm{cmH}_{2} \mathrm{O}$ in extreme inspiration and expiration respectively. ${ }^{5}$ There is no clear evidence in the medical literature on what should be the maximum therapeutic intrapleural negative pressure. ${ }^{3}$ Some authors recommend up to $-40 \mathrm{cmH}_{2} \mathrm{O}$ in some cases, but the therapeutic settings most commonly used seem to range from -10 to $-30 \mathrm{cmH}_{2} \mathrm{O}$. It is thought that higher suction settings generating substantial negative IPP can potentially worsen a pleural leak or even trap lung parenchyma in chest tube holes, leading to lung injury. From an overall perspective, a target maximum IPP of $-30 \mathrm{cmH}_{2} \mathrm{O}$ seems reasonable.

Assuming this, and using our custom PVR device, we could recommend using the Pleur-Evac A-6000 ICC drain at rates of compression up to $60 \mathrm{kPa} \cdot \mathrm{min}^{-1}$ with the suction regulator set at a maximum of $-20 \mathrm{cmH}_{2} \mathrm{O}$. In this setting, the IPP will reach, on average, a maximum of $-31 \mathrm{cmH}_{2} \mathrm{O}$ for less than 30 seconds, before returning progressively back to the set value, and staying in the safe pressure range (Figure 4). However at compression rates beyond $60 \mathrm{kPa} \cdot \mathrm{min}^{-1}$, an initial increase in the negative IPP to greater than $-30 \mathrm{cmH}_{2} \mathrm{O}$ was observed. To minimise the risk of an undesirable increase in negative pressure, and to maintain a more stable IPP throughout the HBOT, compression rates of between 10 and $30 \mathrm{kPa} \cdot \mathrm{min}^{-1}$ should be used. At these rates, the IPP varies no more than -5 $\mathrm{cmH}_{2} \mathrm{O}$ and remains very stable throughout the compression period (Figure 3).

The simulation of an air leak did not compromise the function of the PVR device, suggesting that if the patient were to have a bronchopleural fistula, the use of our device under hyperbaric conditions should still ensure delivery of the necessary ICC suction. In practice, it would be rare for a patient with an air leak of $5 \mathrm{~L} \cdot \mathrm{min}^{-1}$ to undergo HBOT 
rather than to receive surgical intervention before therapy. In comparison with the pressure curves without a simulated pleural air leak, the IPP was more stable when a pleural air leak was simulated (Figure 3). This is presumably due to the air leak playing the same role as our PVR device in ensuring unidirectional airflow through the PDU during compression, preventing generation of excessive negative pressure as the air in the collection chamber is compressed.

The choice of sterile air filter was critical to the development of the device. Many different types of filters (PTFE, PES, PVDF, etc.) with different properties (hydrophobic, hydrophilic), pores sizes (in $\mu \mathrm{m}$ ) and filter surface areas (in $\mathrm{mm}$ diameter) are available commercially. Our final selection was based on a compromise between good filtration properties and the necessary flow resistance to permit enough ambient air to enter into the PDU collection chamber to modulate negative pressure during compression and the maintainance of a safe bacterial filtration capability and avoiding an excessive air leak. A $25 \mathrm{~mm}$ filter with an hydrophobic PTFE membrane with pore size of $1 \mu \mathrm{m}$ ensures good anti-bacterial and anti-viral properties..$^{6,7}$ The PALL filter selected is primarily designed for use with liquids and the filtration capabilities of such membranes are about ten times more efficient in air than in liquid owing to electrostatic charges in the membranes that attract airborne particles and organisms at the surface of the membrane and also to the walls of the pores. Secondly, the pore size is sufficiently small that Brownian motion becomes relevant, with small particles not travelling on straight trajectories when carried in air, making it difficult for them to progress through the thickness of a filter without becoming trapped in the matrix of the filter. ${ }^{6}$

There are some caveats on the use of the PVR device in the clinical setting. In particular, the PDU must be connected to the chamber's suction system and confirmed to be under suction before the PVR can be connected. While the controlled leak from the PVR is small, the application of the device prior to the application of suction to the PDU could nevertheless result in the patient's lung collapsing. Similarly, the device must be removed from the patient-tube prior to the PDU being removed from the chamber suction system at the end of the treatment. With appropriate staff training and protocols in place, this has not proven to be an issue in the clinical setting in our institution.

\section{Conclusions}

The use of a PDU under hyperbaric conditions normally requires significant intervention from staff and reduced compression rates to prevent an unacceptable increase in IPP during variations in ambient pressure. While the PleurEvac A-6000 ICC drain can be used safely in hyperbaric chambers without suction (during compression) and at a maximum compression rate of $10 \mathrm{kPa} \cdot \mathrm{min}^{-1}$, the use of our PVR device allows for the IPP to remain within an acceptable range without intervention from the staff during all phases of HBOT, including at compression rates up to $60 \mathrm{kPa} \cdot \mathrm{min}^{-1}$ with continuation of pleural suction at settings up to -20 $\mathrm{cmH}_{2} \mathrm{O}$. For routine treatments, we would recommend a compression rate of $30 \mathrm{kPa} \cdot \mathrm{min}^{-1}$, where the generated IPP is most stable. Finally, it is important to remember that during decompression it is highly desirable for any ICC drain to be on suction in order to avoid the complication of an expanding pneumothorax. A critical practice point is that if the PVR device is to be used, the PDU must be on suction before it is attached and the device removed before taking the patient off suction for transport.

\section{References}

1 Bar-El Y, Ross A, Kablawi A, Egenburg S. Potentially dangerous negative intrapleural pressures generated by ordinary pleural drainage systems. Chest. 2001;119:511-4.

2 Baumann MH, Patel PB, Roney CW, Petrini MF. Comparison of function of commercially available pleural drainage units and catheters. Chest. 2003;123:1878-86.

3 Manzanet G, Vela A, Corell R, Moron R, Calderon R, Suelves C. A hydrodynamic study of pleural drainage systems: some practical consequences. Chest. 2005;127:2211-21.

4 Walker KJ, Millar IL, Fock A. The performance and safety of a pleural drainage unit under hyperbaric conditions. Anaesth Intensive Care. 2006;34:61-7.

5 Munnell ER. Thoracic drainage. Ann Thorac Surg. 1997;63:1497-502.

6 Leahy TJ, Gabler R. Sterile filtration of gases by membrane filters. Biotechnol Bioeng. 1984;26:836-43.

7 Olson WP, Vanden Houten L, Ellis JE. Sterile vent filter function test. J Parenter Sci Technol. 1981;35:70-1.

\section{Acknowledgements}

The authors thanks the team of The Alfred Hyperbaric Medicine Unit for its support and patience.

\section{Conflicts of interest and funding: nil}

Submitted: 22 November 2015; revised 15 March and 17 July 2017 Accepted: 19 July 2017 


\title{
Case reports \\ Periorbital emphysema after a wet chamber dive
}

\author{
Wei-Shih Tseng ${ }^{1,2}$, Hui-Chieh Lee ${ }^{1}$, Bor-Hwang Kang ${ }^{1,3}$ \\ ${ }^{1}$ Department of Diving and Hyperbaric Medicine, Zuoying Branch of Kaohsiung Armed Forces General Hospital, Kaohsiung, \\ Taiwan \\ ${ }^{2}$ Department of Neurology, Tri-Service General Hospital, National Defense Medical Center, Taipei, Taiwan \\ ${ }^{3}$ Department of Otorhinolaryngology Head and Neck Surgery, Kaohsiung Veterans General Hospital, Kaohsiung, Taiwan
}

Corresponding author: Bor-Hwang Kang, Associate Professor and Director of Rhinology, Department of Otorhinolaryngology Head \& Neck Surgery, Kaohsiung Veterans General Hospital, 386 Dazhong 1st Rd, Kaohsiung, Taiwan 81362

bhkang@vghks.gov.tw

Key words

Barotrauma; Diving medicine; Military diving; Risk factors; Simulation; Training; Valsalva manoeuvre

\begin{abstract}
(Tseng W-S, Lee H-C, Kang B-H. Periorbital emphysema after a wet chamber dive. Diving and Hyperbaric Medicine. 2017 September;44(3):198-200.)

Although periorbital emphysema (PE) is commonly associated with orbital fractures, it may develop without any fracture or significant trauma in circumstances such as post-surgery, infection, forceful nose blowing, sneezing, and weight lifting. We report on a healthy military diver who developed PE following a wet chamber dive. A diagnosis of PE secondary to sinus barotrauma was reached. He was treated conservatively without medication and his symptoms recovered completely within 10 days. To the best of our knowledge, only five cases of diving-related PE have been reported in the literature. Analysis of these cases and ours revealed that facial trauma, repeated forceful Valsalva manoeuvres and recent upper respiratory tract infection are probable risk factors for diving-related PE.
\end{abstract}

\section{Introduction}

Before their training/missions in the open sea, navy divers receive training in the wet-and-dry recompression chamber at the Diving Medical Centre of the Zuoying Branch of Kaohsiung Armed Forces General Hospital. Divers are immersed in the water within the chamber and the pressure is increased to simulate descending in the water. This training process allows divers to become familiar with diving sequences and with their equipment in a monitored and relatively safe environment. The medical problems that could occur during diving include middle ear barotrauma, sinus barotrauma, oxygen toxicity, pulmonary barotrauma, air embolism, and decompression sickness. ${ }^{1}$ Here we describe the case of a diver who developed periorbital emphysema (PE) immediately after a simulated dive.

\section{Case report}

A healthy, 26-year-old, experienced, male military diver without a previous history of smoking, rhinitis, sinusitis or barotrauma had a scheduled wet training dive to a simulated depth of 36.6 metres' sea water (msw). The diver experienced difficulty in equalizing pressure in both ears during descent but managed to equalize middle ear pressure by the Valsalva manoeuvre and reached a simulated depth of $12.2 \mathrm{msw}$. However, gradual development of a sensation of pressure over the left medial orbital region was noted and he asked to abort the dive. Pain and swelling of the left periorbital region developed immediately after surfacing. Ptosis and mild ecchymosis around the left orbit were also noted (Figure 1). On examination, ocular movements, pupillary light reactions and dilated fundus examination were normal. There was a non-tender left periorbital swelling with crepitus on palpation. An antero-posterior X-ray view of the head showed air around the left superior orbital margin (Figure 2). Coronal computed tomography (CT) imaging of paranasal sinuses revealed multiple air bubbles in the subcutaneous tissue of the left periorbital region (Figure 3). No definitive bone fracture could be identified in this region. PE secondary to sinus barotrauma was diagnosed. Reviewing his history, there was a recent episode of upper respiratory tract infection (URTI), which is likely the main contributing factor leading to his difficulty equalising, sinus barotrauma and the associated PE. The patient was treated conservatively without medication and advised to avoid diving for one month. His symptoms gradually subsided with complete resolution after 10 days. There has been no recurrence on return to diving for up to one year of follow-up. 
Figure 1

Peri-orbital emphysema secondary to sinus barotrauma after finishing a simulated wet-chamber dive; swollen left eye and palpable subcutaneous emphysema

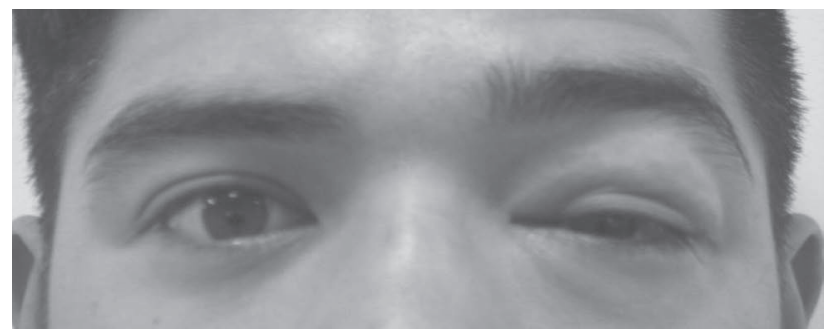

Figure 3

A coronal plane of CT scan showing air in left orbit (arrows)

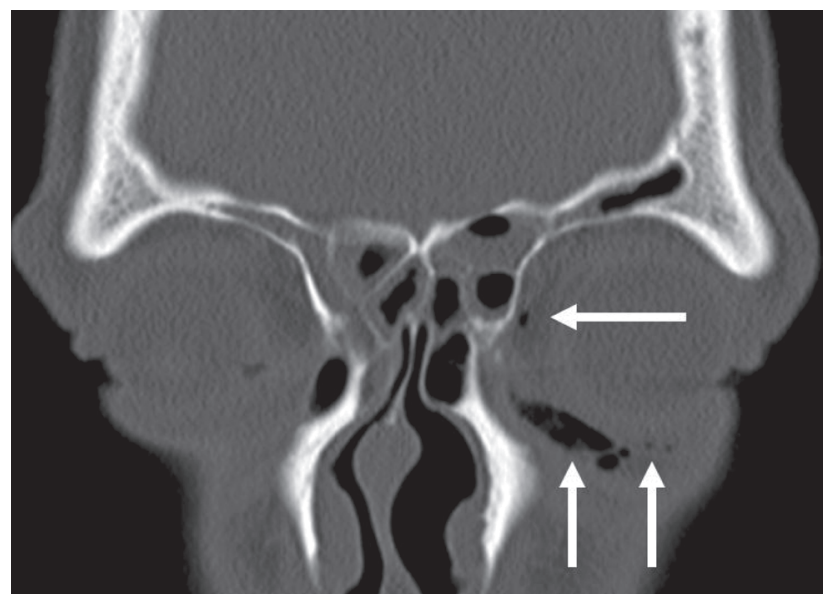

Figure 2

Radiograph of orbit, showing air in superior left orbit (black eyebrow sign, arrows)

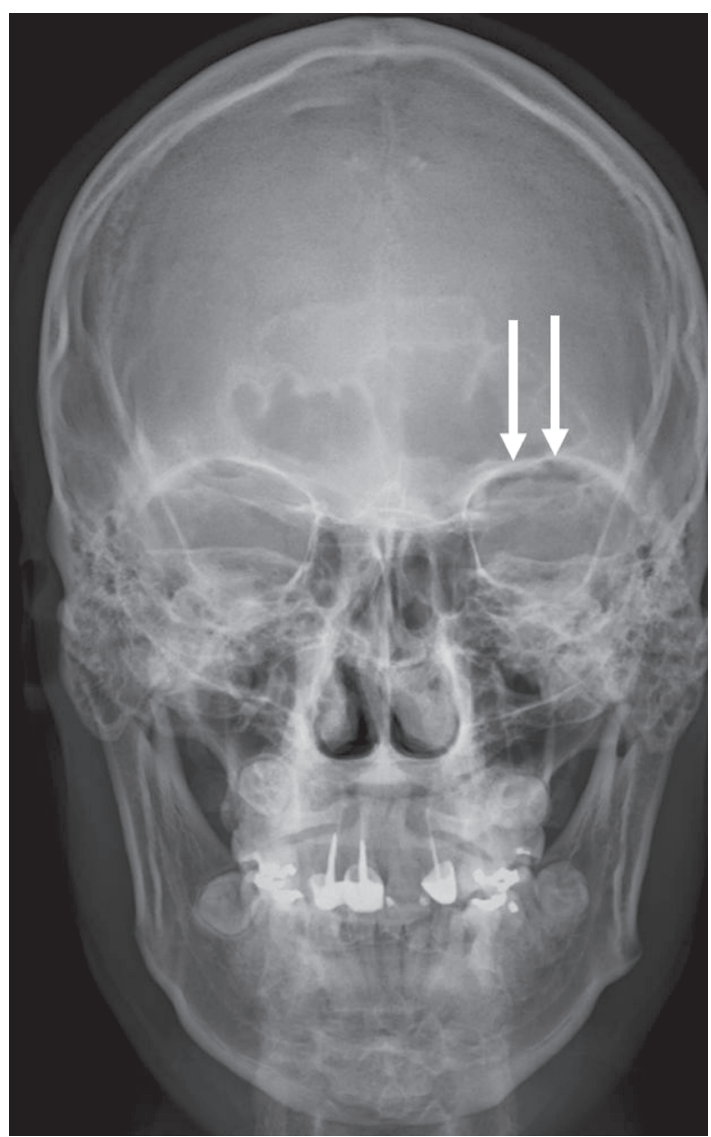

\section{Discussion}

$\mathrm{PE}$ is a relatively uncommon condition in which air is present within the eyelids or orbit. ${ }^{2}$ Periorbital air most commonly comes from a fracture or perforation of one of the orbital bones due to direct or indirect trauma. ${ }^{3}$ Frequently, this fracture involves the fragile part of the medial orbital wall, the lamina papyracea. ${ }^{2,3}$ Other mechanisms include postsurgery, infection, forceful nose blowing, sneezing, weight lifting, bungee jumping and pressure changes during airplane travel or free diving, as a complication of sinus barotrauma. ${ }^{4}$ To the best of our knowledge, there are only five previously reported cases of diving-related PE (Table 1). ${ }^{4-7}$ All of these cases, except one that was not documented, were male. Images with radiography or CT showed no bony fracture. Recovered was complete within two weeks. According to these articles, facial trauma, repeated forceful Valsalvas, and recent URTI were probable risk factors for PE.

In our diver, we presume that increased intranasal pressure as a result of repeated and forceful Valsalvas caused a tear in the thin medial orbital wall. In accordance with Boyle's law, the volume of air within the orbit expanded proportionally to the decrease in pressure during ascent, further exaggerating his left PE.
The diagnosis of PE is usually made by patient history and physical findings, confirmed with orbital CT. Although CT is the primary imaging modality for evaluating patients with trauma, conventional radiography is often the first and valuable imaging study performed. For intra-orbital air, mostly caused by orbital fracture, air rises into the superior side of the orbit in a linear fashion, simulating a black eyebrow. The black eyebrow sign on plain radiography is very useful and easily observed for physicians working in the emergency department. ${ }^{8}$

$\mathrm{PE}$ is generally a benign, self-limiting condition, as the air is absorbed within two weeks. ${ }^{2,9}$ The role of antibiotic prophylaxis in PE remains controversial. Administration of an antibiotic might be considered in cases with a concomitant URTI such as acute sinusitis., ${ }^{2,3}$ Rarely, tension PE can lead to visual loss due to retinal artery occlusion and optic nerve ischaemia. $^{2}$ Thus, immediate ophthalmologic examination and treatment are warranted if the patient presents with a painful eye and visual changes. Additionally, patients need to be educated to avoid activities that may raise intranasal pressure or allow expansion of air within the orbital soft tissues such as nose blowing, sneezing, diving, flying, or climbing to high altitudes for at least two weeks. ${ }^{9}$ 


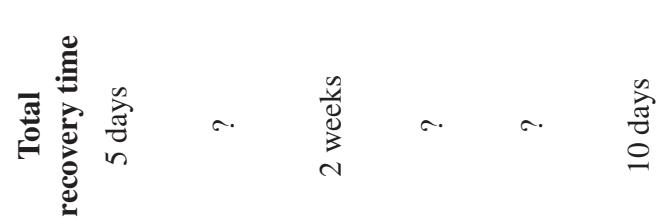

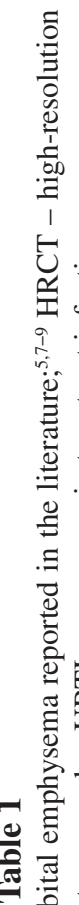

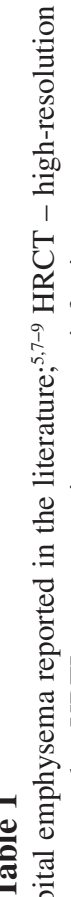

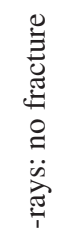

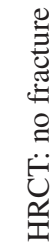

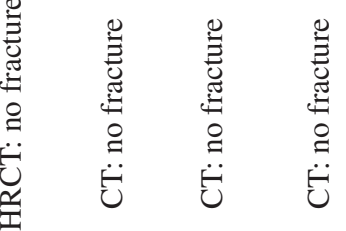

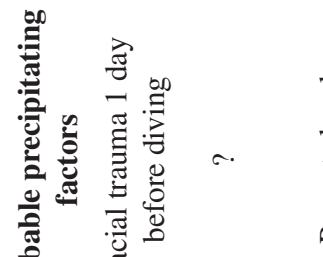

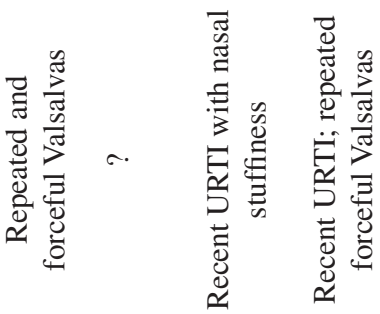

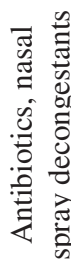

î

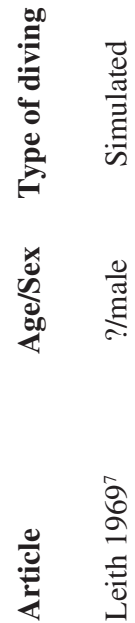

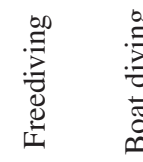

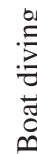

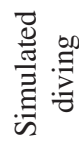

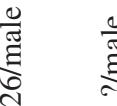

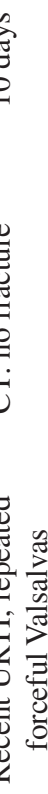

$\stackrel{\Xi}{Z}$

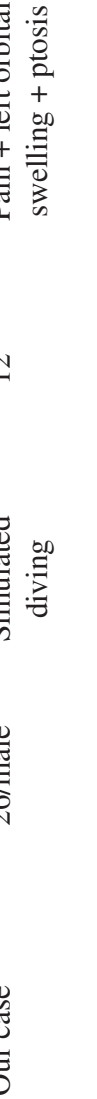

In conclusion, diving complications can occur even in the monitored and relatively safe environment of a recompression chamber. Physicians/diving instructors should always keep in mind the importance of a careful evaluation of trainees before committing them to a diving task or training programme. Divers should be aware of the risk factors related to middle ear/sinus barotrauma and report their discomfort and terminate diving in a timely fashion to avoid major diving complications.

\section{References}

1 Bove AA. Diving medicine. Am J Respir Crit Care Med. 2014;189:1479-86.

2 Zimmer-Galler IE, Bartley GB. Orbital emphysema: case reports and review of the literature. Mayo Clin Proc. 1994;69:115-21.

3 Mohan B, Singh KP. Bilateral subcutaneous emphysema of the orbits following nose blowing. J Laryngol Otol. 2001;115:319-20.

4 Pennell DJ, Asimakopoulos P, Ram B, Veitch DY. Periorbital emphysema after dive barotrauma without radiological evidence of paranasal sinus injury. Aviat Space Environ Med. 2014;85:863-6.

5 Leitch DR. Unusual case of emphysema. Br Med $\mathrm{J}$. 1969;1:383.

6 Bolognini A, Delehaye E, Cau M, Cosso L. Barotraumatic orbital emphysema of rhinogenic origin in a breath-hold diver: a case report. Undersea Hyperb Med. 2008;35:163-7.

7 Hall JE. 'Popeye the Sailor': facial emphysema after a surface-supplied air dive. BMJ Case Rep. 2013;2013:bcr2013009928. doi:10.1136/bcr-2013009928.

8 Feyaerts F, Hermans R. The black eyebrow sign in orbital blowout fracture. JBR-BTR. 2009;92:251-2.

9 Seiff SR. Perspective: atmospheric pressure changes and the orbit: recommendations for patients after orbital trauma or surgery. Ophthal Plast Reconstr Surg. 2002;18:239-41.

\section{Acknowledgements}

Written consent was obtained from the patient to publish details of his case.

Funding and conflicts of interest: nil

Submitted: 17 July 2017; revised 18 July 2017

Accepted: 19 July 2017 


\title{
Ruptured tympanic membrane from underwater impact with an Atlantic salmon, Salmo salar
}

\section{David Smart}

Department of Diving and Hyperbaric Medicine, Royal Hobart Hospital and School of Medicine, and Faculty of Health Sciences, University of Tasmania, GPO Box 1061, Hobart, Tasmania 7001, Australia

david.smart@ths.tas.gov.au

\section{Key words}

Ear barotrauma; Underwater hazards; Marine animals

\begin{abstract}
(Smart D. Ruptured tympanic membrane from underwater impact with an Atlantic salmon, Salmo salar. Diving and Hyperbaric Medicine. 2017 September;47(3):201-202.)

An unusual case of tympanic membrane rupture in a diver due to impact from an Atlantic Salmon, salmo salar is described. The diver was treated conservatively and the injury fully healed by eight weeks after which the diver successfully returned to diving. The author contends that the appearances of such traumatic perforations differ from barotrauma in divers secondary to ear clearing problems on descent.
\end{abstract}

\section{Introduction}

Ear barotrauma is a common injury in compressed gas divers and snorkellers. Middle ear barotrauma (MEBt) is a subset of ear barotrauma and is the most common medical disorder experienced by divers, constituting $46 \%$ of diving ear, nose and throat disorders. ${ }^{1,2}$ It results from inability to auto-inflate the middle ear to match the outside pressure as it rises when the diver descends. The tympanic membrane (TM) is stretched beyond physiological limits sustaining injury. The MEBt injury presents as a spectrum from symptoms of blockage (but no signs), through progressively increasing inflammation in the middle ear and of the TM, which frequently is accompanied by an effusion. ${ }^{1}$

The most extreme form of MEBt is a perforation of the TM. Exact figures are unknown, but perforated TM is quite rare in divers compared with other grades of MEBt, probably because they experience intolerable pain and abort their dive before perforation occurs. In addition, effusion and middle ear mucosal swelling may dampen the degree of force on the TM, protecting it from the extreme deviation that produces perforation. If a perforation does occur in this setting, it may be subtle and difficult to diagnose. Divers exposed to a rapid descent (for example, accidental over-weighting) or a sudden external pressure force (for example, a blast injury) may also present with TM perforation. Three cases have been reported anecdotally from swim fin blows to the side of the head, despite water-polo cap ear protection, during underwater hockey competition (Davis FM, personal communication, 2017). It is believed this is the first reported case of TM rupture due to direct impact from the Atlantic salmon, Salmo salar (presented with the patient's permission).

\section{Case report}

A 34-year-old male occupational diver was undertaking his usual duties as a diver on an Atlantic salmon farm in Tasmania, Australia. He was wearing an $8 \mathrm{~mm}$ wetsuit with full neoprene hood, breathing from surface supply breathing apparatus with communications. He had completed a number of dives within the salmon pens as part of his routine work. He had no ear clearing problems or symptoms during his dives. He was submerged at a depth of 17 metres at the base of the fish enclosure when he noticed that the salmon were unusually active and swimming rapidly around him. A fish of approximately $5 \mathrm{~kg}$ in mass collided with the right side of his head over his right ear, causing him immediate pain, tinnitus and a brief feeling of vertigo. He communicated the event to his supervisor then commenced a slow ascent.

On arrival at the support vessel, his vertigo had improved, but he had persistent pain, tinnitus and impaired hearing in the right ear. He was initially assessed by his local GP, who referred him to the Royal Hobart Hospital hyperbaric facility. Otoscopic examination demonstrated a normal left tympanic membrane (TM), with normal auto-inflation. The right $\mathrm{TM}$ demonstrated a small $(<25 \%)$ perforation in the lower pars tensa (Figure 1). The otoscope fogged when a Valsalva manoeuvre was attempted, and tympanometry persistently recorded 'open' without producing a pressure curve. Audiometry demonstrated minor loss $(10 \mathrm{~dB})$ in high frequencies compared to his last fitness-to-dive assessment.

The diver was treated conservatively with analgesia and advised not to dive for eight weeks and to keep the ear dry. During this period, he undertook surface-based activities for his employer. Eight weeks later, he was asymptomatic with 
Figure 1

Right tympanic membrane showing perforation in the pars tensa

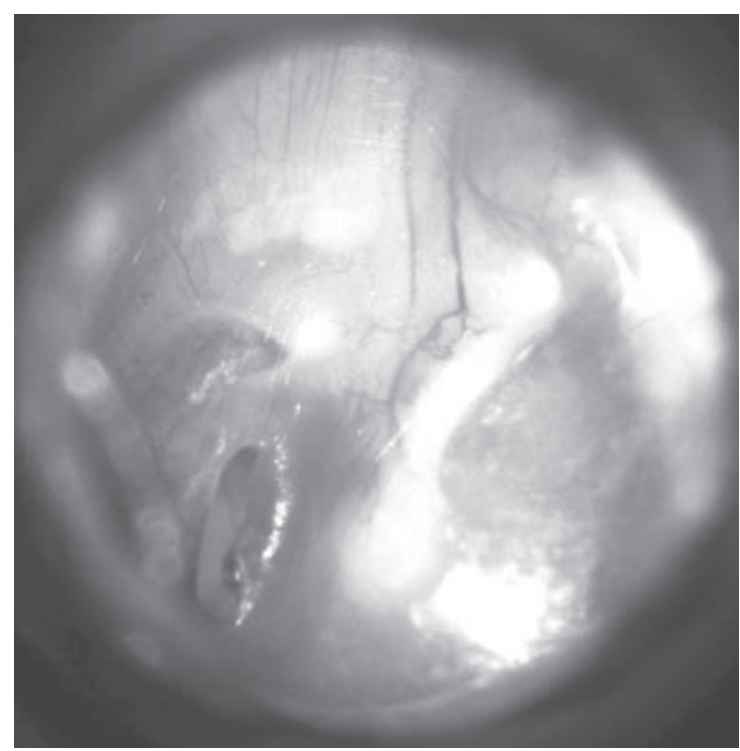

Figure 2

Healed right tympanic membrane at eight weeks post injury

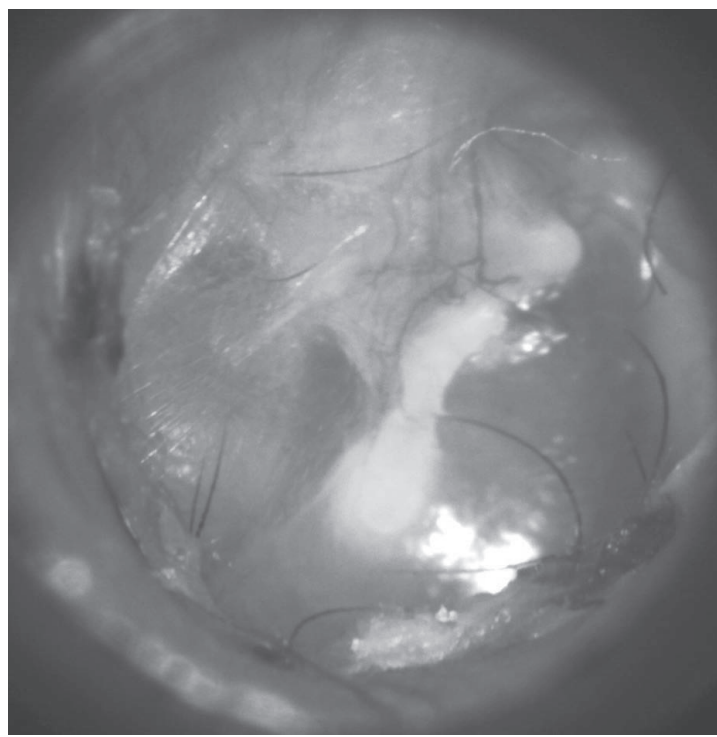

normal looking TMs bilaterally (Figure 2). Autoinflation, tympanometry and audiometry were normal. He has successfully returned to diving without problems.

\section{Discussion}

Traumatic TM rupture can cause severe pain, bleeding, tinnitus and hearing loss for the injured individual. ${ }^{1-3}$ Other than diving, TM perforation can result from multiple aetiologies. These can include direct penetrating trauma from foreign objects, percussion injury (slaps, water ski accidents), sudden negative pressure, basal skull fracture, barotrauma or iatrogenic injury. 3,4 Larger perforations appear to result from higher energy injuries or direct penetrating trauma. The following factors affect prognosis for healing: large perforations are less likely to heal spontaneously; final healing and hearing results are good regardless of treatment employed; spontaneous healing provides a better result than surgical intervention and infection adversely affects healing. ${ }^{4}$ "Masterly inactivity should be religiously followed and unnecessary surgical intervention should be discouraged" when assessing treatment options. ${ }^{3}$ Between $80 \%$ and $90 \%$ of perforations heal spontaneously inside three months. ${ }^{4-6}$ Use of gel-foam patches to treat larger tears has been reported to improve healing in a randomised controlled trial. ${ }^{6}$

In my experience of more than twenty cases of perforated TMs from diving, the localised pattern of perforation from Salmo salar differs from TM injury sustained by divers when they cannot clear their middle ear, resulting in MEBt. Divers with ear-clearing problems tend to have more widespread injection of the TM associated with the perforation. The perforation is usually less obvious (often a small slit), or may be hidden by haemorrhage, and requires confirmation by tympanometry. I postulate that this latter injury is due to slower onset of the barotrauma, which may follow multiple (unsuccessful) attempts at clearing the ears. I have been unable to locate any reported cases of tympanic membrane rupture due to direct impact from the Atlantic Salmon, Salmo salar, or other fish.

\section{References}

1 Dysbaric diseases: barotrauma. In: Edmonds C, Bennett M, Lippmann J, Mitchell S, editor. Diving and subaquatic medicine, 5th ed. Boca Raton, FL: CRC Press; 2015. Print ISBN: 978-1-4822-6012-0; eBook ISBN: 978-1-4822-6013-7. Available from: https//doi.org/10.1201/b18700.

2 Klingmann CI, Praetorius M, Baumann I, Plinkert PK. Otorhinolaryngologic disorders and diving accidents: an analysis of 306 divers. Eur Arch Otorhinolaryngol. 2007;264:1243-51.

3 Wani A, Rehman A, Lateef S,Malik R, Ahmed A, Ahmad W, Kirmani M. Traumatic tympanic membrane perforation: an overview. Indian Journal of Otology. 2016;22:100-4.

4 Griffin WL. A retrospective study of traumatic tympanic membrane perforations in a clinical practice. The Laryngoscope. 1979;89:261-82. doi:10.1288/00005537-197902000-00009

5 Orji FT Agu CC. Determinants of spontaneous healing in traumatic perforations of the tympanic membrane. Clin Otolaryngol. 2008;33:420-6. doi:10.1111/j.17494486.2008.01764.x

6 Lou Z-C, Tang Y-M, Yang J. A prospective study evaluating spontaneous healing of aetiology, size and type-different groups of traumatic tympanic membrane perforation. Clin Otolaryngol. 2011;36:450-60. doi:10.1111/j.17494486.2011.02387.x

\section{Funding and conflicts of interest: nil}

Submitted: 01 May 2017; resubmitted 10 July 2017

Accepted: 10 July 2017 


\section{Letters to the Editor}

\section{Proliferative retinopathy during hyperbaric oxygen treatment}

A 43-year-old male with type 2 diabetes mellitus, treated with insulin for 28 years and with an $\mathrm{HbA1c}$ of $7.9 \%$ six months prior, suffered from bilateral plantar ulcers refractory to specialised wound care. He underwent a planned 40 sessions of hyperbaric oxygen treatment (HBOT) at $243 \mathrm{kPa}$ for 90 minutes. Consent was given for this report.

The patient's ophthalmic history included bilateral proliferative diabetic retinopathy (PDR) identified on routine diabetic eye screening three years previously. This was treated with pan-retinal photocoagulation (PRP). Three months before starting HBOT, he underwent phacoemulsification and intra-ocular lens insertion of his left eye, having had the same procedure done to his right eye a year prior, without complication. He was reviewed again one week prior to his first HBOT and fundoscopy confirmed non-proliferative diabetic retinopathy (NPDR) without evidence of PDR.

The patient had a routine follow up by the ophthalmologist following his fifth HBOT when fundoscopic examination revealed pre-retinal haemorrhage, a form of PDR, in his left eye. This was treated with PRP at the time. His visual acuity, 6/9 bilaterally, had not changed, nor did he describe any changes in his visual field despite these findings.

He was seen three weeks later (following 12 further HBOT) when fundoscopy showed worsening proliferative changes, this time in both eyes. Bevacizumab was injected at the time and fill-in PRP performed the following week. His visual acuity remained unchanged in both eyes. At this point, HBOT was withheld to allow the proliferative phase of the patients' retinopathy to remit.

The potentially adverse effects of hyperbaric oxygen to the retinal vasculature of diabetic patients was postulated in 1994 following a similar experience, albeit without a baseline fundoscopic examination. ${ }^{1}$ In particular, the concern was of accelerating the proliferative process of retinopathy with subsequent irreversible loss of vision. Thereafter, routine screening and treatment of all diabetic patients for PDR was adopted at our facility. Until now, there have been no further cases of NPDR evolving into PDR at three-month review following HBOT. Indeed, a brief literature search using the terms "retinopathy", "complications", "adverse effects", "vitreous", "hemorrhage", "haemorrhage", "hyperbaric" and "oxygen" has not found any other cases described.

In a double blind, randomised trial (meeting abstract only) of 15 diabetic patients with both NPDR and PDR, patients in neither the HBOT (243 kPa for $90 \mathrm{~min}$ ) nor the control arm had evidence of neovascularisation nor worsening of their proliferative retinopathy at three-month follow up. ${ }^{2}$
The significance of PDR following cataract surgery has also been considered. A review article consistently found that NPDR progression occurred in up to a third of such patients. Despite this, there were no cases of NPDR progressing to PDR at 12-month follow up. ${ }^{3}$

Whether this patient's sudden progression to PDR was related to HBOT, recent cataract surgery or another unknown factor is unclear. However, the temporal relationship to 17 HBOT is difficult to explain and appears more rapid than available data regarding vascular regrowth in wound healing would suggest.

\section{References}

1 McCartney P, McCartney P. Vitreous haemorrhage after hyperbaric oxygen therapy. Eye. 1994;8:705-6.

2 Jalabi MW, Abidia A, Kuhan G, Laden G, Verma D, McCollum PT. The safety and effect of hyperbaric oxygen therapy in patients with diabetic retinopathy - a double-blind randomised controlled trial [Abstract]. Undersea Hyperb Med. 2001;28(Suppl):99.

3 Mohammed A, Ali K, Soliman T. Effect of cataract surgery on diabetic retinopathy. Benha Medical Journal. 2015;32:92.

Viet Tran ${ }^{1,2}$, David Smart ${ }^{1,2}$

${ }^{1}$ Hyperbaric Medicine Unit, Royal Hobart Hospital, Tasmania, Australia

${ }^{2}$ School of Medicine, University of Tasmania, Australia

dr.vtran@gmail.com

\section{Key words}

Hyperbaric medicine; Vision; Side effects; Ophthalmology; Letters (to the editor)

\section{Advertising policy for Diving and Hyperbaric Medicine}

This letter is intended to serve as a policy document in relation to advertising in the journal Diving and Hyperbaric Medicine (DHM). The remarks and suggestions below have the support of both the EUBS and SPUMS, henceforth referred to as the publishers.

The publishers support the concept of advertising in DHM and value highly the contribution that any advertisers would make to the maintenance of a high-quality scientific journal. Nevertheless, it is considered that there are potential advertisers who would not be acceptable to the members of both our societies. These include those designed to market unhealthy or inefficacious products. The categories that are considered to fall under the description of 'unacceptable' are outlined below and to advertise such products and services is regarded as contrary to the scientific integrity of the Journal.

The publishers also note there is a belief in both societies that, while the appearance of an advertisement in DHM does not specifically demonstrate endorsement of the service or product by the Journal or the Societies, such an 
endorsement may be assumed by many readers. There is a distinction in this regard between advertising in a nonscientific publication and a scientific journal.

The publishers recognise the final decision to reject or accept an individual advertiser lies ultimately with the Editor and the Editorial Board. These guidelines are designed to assist in decision making on this subject.

The publishers suggest the following categories of services and products would not be acceptable:

- harmful or illegal products (such as: alcohol, tobacco, recreational drugs or any such product that is classified as illegal or harmful in Australia/New Zealand or the European Community);

- $\quad$ products and/or services in the field of hyperbaric and diving medicine that can be classified as "non-justified use' by the criteria set forth respectively by SPUMS and EUBS/ECHM in their official publications;

- $\quad$ products or services that compromise diving safety;

- various 'alternative' forms of oxygen administration (e.g., topical HBO, mild hyperbaric therapy);

- marketing the use of 'non-approved' indications (category D, E, F in the ECHM list of indications; anything not on the SPUMS list);

- marketing in support of a product or service without a medically justifiable intent. This includes any drug or physical intervention designed to increase performance or which can potentially place patients at an unacceptable risk (such as doping products);

- products and/or services where there is no relationship to diving, diving and hyperbaric medicine, emergency, intensive care or related fields of medicine and where the same 'non-justified use' criteria apply as above;

- any product and/or service where there is a material conflict of interest with the Editor or any member of the Editorial Board. Consideration may be given to accepting an advertisement if there is a declaration of such a conflict and the Board member concerned is not involved in any decisions concerning placement, fees charged or accompanying articles.

The Editor of DHM may refer any requests for advertising to the Journal Governance Committee for advice and clarification as required.

Jacek Kot, President, EUBS <jacek.kot@eubs.org>

David Smart, President, SPUMS<president@spums. org.au>

On behalf of the publishers EUBS and SPUMS

\section{Key words}

Policy; Medical society; Diving industry; Hyperbaric medicine; Letters (to the Editor)

Diving and Hyperbaric Medicine Journal website is at

\section{<www.dhmjournal.com>}

Articles for immediate release into the public domain, information about submitting to the Journal, profiles of the Editorial Board and contents of the most recent and previous issues are to be found on the site. 


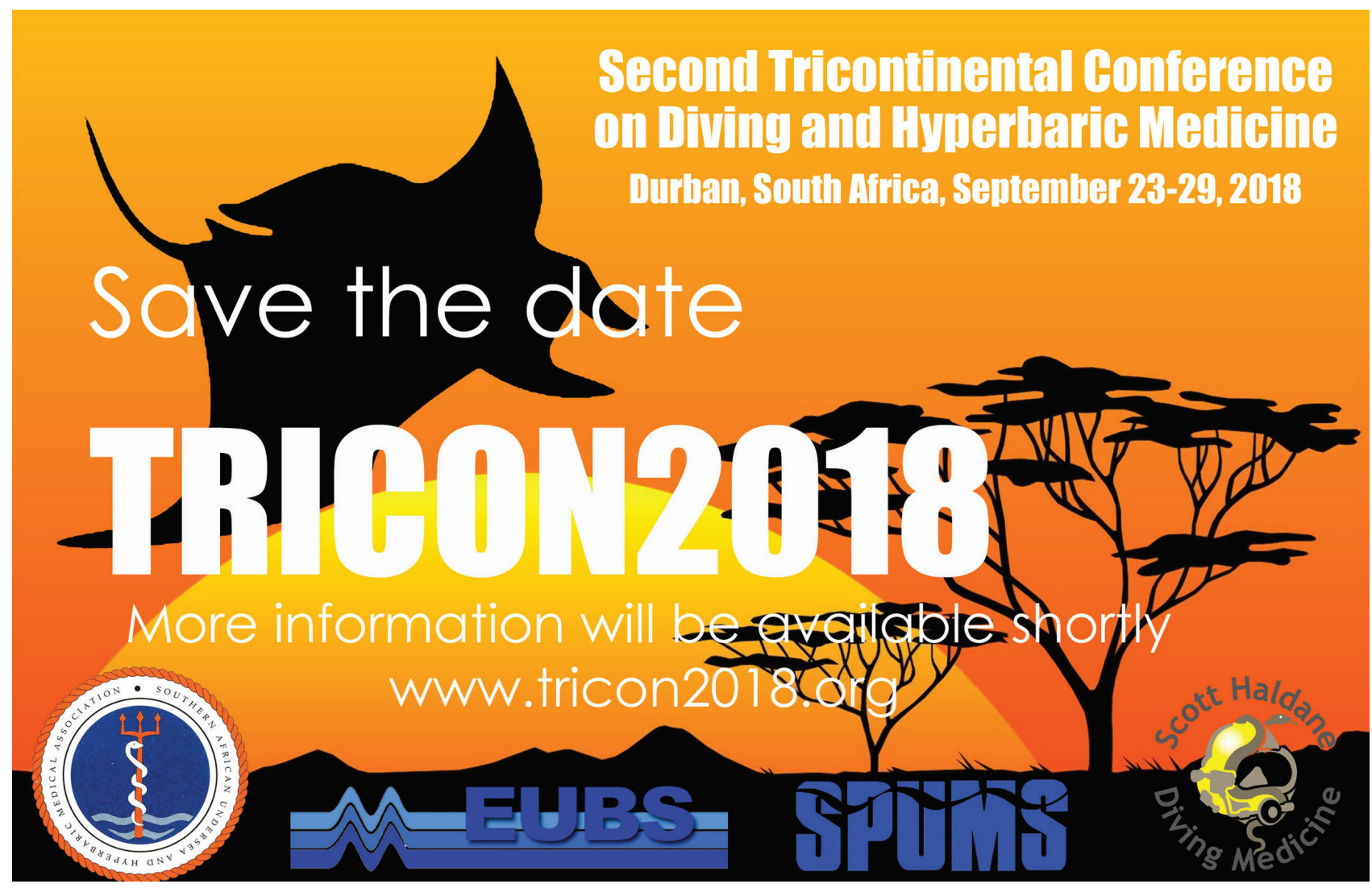

\title{
Second Tricontinental Scientific Conference on \\ Diving and Hyperbaric Medicine www.tricon2018.org
}

\author{
Dates: 23-29 September 2018 \\ Venue: Durban, South Africa
}

After a very successful first edition in 2013 on Reunion Island, our next Tricontinental Scientific Conference will take place in the coastal city of Durban, KwaZulu Natal, South Africa.

We are once again organising a full week with scientific days interspersed between diving workshops and social events. The academic programme will include presentations, workshops, discussion sessions and special topic conferences. Our social calendar encompasses a welcome cocktail party, a gala dinner and the ever popular post-conference happy hour. There will be opportunities to dive the nearby Aliwal Shoal, visit wildlife game parks, take in a local rugby match and explore Zulu culture.

A Joint Organising Committee from EUBS, SPUMS, SAUHMA and the Scott Haldane Foundation will work together with local Durban Hyperbaric Centre staff and a South Africa Event Management Bureau to make sure everything runs smoothly.

The combination of easy access, friendly people, rich culture, nature at its most spectacular and affordable prices makes this an opportunity not to be missed. The weather in September is ideal with temperatures in the low 20s for both land and sea and little chance of rain. Why not plan an extra week before or after the conference to travel the area and experience more of South Africa's amazing diversity, hospitality and wildlife. Bring your family too - there are a lot of child-friendly activities nearby!

Visit the dedicated website: <www.tricon2018.org $>$ for all information and to start planning your trip. We very much look forward to seeing you there. 


\section{Notices and news}

\section{EUBS notices and news and all other society information is now to be found mainly on the society's website: <www.eubs.org>}

\section{EUBS Member at Large}

As the EUBS Annual Scientific Meeting is being held in September, EUBS membership will have elected a new Member-at-Large and our 2014 Member-at-Large, Professor Rob van Hulst, will have left the Executive Committee. We would like to thank him for his support and help and hope to be able to count on him in the future!

\section{ECHM Level 3 (Expert in Hyperbaric Medicine) recognition}

On 23 May 2017, the Executive Board of the European Committee for Hyperbaric Medicine (ECHM) certified the following Level 3 Hyperbaric Medicine Experts, based on the grandfathering process as described in the ECHMEDTC Educational and Training Standards for Diving and Hyperbaric Medicine, 2011. This is the highest level of recognition in hyperbaric medicine expertise, and testifies to the individuals' long-time continued efforts in our field. As most are active members of EUBS, we would like, on behalf of EUBS, to congratulate them warmly.

Pieter Bothma, UK

Philip Bryson, UK

Maide Cimsit, Turkey

Ramiro Cali Corleo, Malta

Jordi Desola Ala, Spain

Peter Germonpré, Belgium

Mark Glover, UK

Karin Hasmiller, Germany

Eric Christian Jansen, Denmark

Jacek Kot, Poland

Folke Lind, Sweden

Pasquale Longobardi, Italy

Alessandro Marroni, Italy

Daniel Mathieu, France

Roswitha Prohaska, Austria

Monica Rocco, Italy

Zdzislaw Sicko, Poland

Gunalp Uzun, Turkey

Albert Van den Brink, The Netherlands

Tjeerd van Rees Vellinga, The Netherlands

Wilhelm Welslau, Germany

Jurg Wendling, Switzerland

\section{European Wound Management Association (EWMA) position paper on oxygen therapies}

In May 2017, the EWMA published an official statement on the "Use of oxygen therapies in wound healing". ${ }^{1}$ Collaborating on this paper, on behalf of EUBS and ECHM, were EUBS President, Jacek Kot and Pasquale Longobardi from the hyperbaric centre, Ravenna. This document provides practice-oriented guidance on the current use of various forms of oxygen therapies for wound treatment. While the document considers not only hyperbaric oxygen therapy but also other localised forms of oxygen therapies, it is an important awareness document to clinicians in Europe and beyond. The document was co-opted by Wounds Australia, a charity body for wound care and management in Australia through advocacy, education and research, whose Tasmanian State Lead RN Carol Baines also collaborated in the text. The full document can be downloaded freely from the EWMA website http://ewma.org/what-we-do/ewmaprojects/we-are-currently-working-on/oxygen-therapiesguidance-document/.

\section{Reference}

1 Gottrup F, Dissemond J, Baines, Frykberg R, Jensen P, Kot J, et al. Use of oxygen therapies in wound healing, with special focus on topical and hyperbaric oxygen treatment. $J$ Wound Care, 2017;26 Suppl:S1-S42.

\section{Open Letter to the American Diabetes Association Board of Directors}

The American Diabetes Association has made clear their intention on releasing a Professional Practice Committee statement, recommending against the use of hyperbaric oxygen therapy in diabetic wounds.

"The PPC reviewed the available data on the use of hyperbaric oxygen therapy in 2016 and did not identify enough supporting data on the efficacy of this treatment to recommend its use."

In an unprecedented collaborative effort, the Presidents of major scientific hyperbaric medicine organisations worldwide have responded with an Open Letter to the Board of Directors of the ADA, requesting this statement be withheld and a collaborative discussion based on actual scientific data held before any recommendation is made. This letter (19 July 2017) can be downloaded from the news section of the EUBS website. Please do so and share it with your colleagues! 


\section{Notices and news}

\section{SPUMS notices and news and all other society information is to be found mainly on the society website: <www.spums.org.au>}

\section{Australian and New Zealand College of Anaesthetists news}

The new Diploma of Advanced Diving and Hyperbaric Medicine was launched on 31 July 2017. Those interested in applying for training are directed to the Australian and New Zealand College of Anaesthetists website: <www.anzca.edu. $\mathrm{au} /$ training/diving-and-hyperbaric-medicine>

The curriculum and handbook for training can be found there, as well as documents for units wishing to apply for accreditation.

The process for application for transition credits for those transitioning from the Certificate in DHM to the Diploma closed on 28 August 2017. Those who did not apply within that timeframe can apply for recognition of prior learning. All queries should be directed to <dhm@anzca.edu.au.>

Suzy Szekely

Chair, ANZCA Diving and Hyperbaric Medicine Special Interest Group

suzy.szekely@health.sa.gov.au

\section{Publications database of the German Diving and Hyperbaric Medical Society (GTUeM)}

One of the new resources available to SPUMS members since the launch of the new website is access to the German Society's very large database of publications in diving and hyperbaric medicine. This is a special offer for SPUMS members and will enhance anyone's literature search. EUBS members have had this access for many years.

Access to the database is simple. Members should log in with their user name and password, click on "Resources" then on "GTUeM database" in the pull-down menu. This opens a new window; click on the link provided and enter the user name and password listed on the page which will then let you access the database. Much of the SPUMS Journal and all of Diving and Hyperbaric Medicine up till early 2016 is now on this database as individual, downloadable articles.

The SPUMS Executive wishes to thank the GTUeM for allowing our members access to their collection of diving and hyperbaric medicine publications, which now runs into many thousands.

\section{SPUMS Website News}

The website continues to evolve under the diligent attention of the Web Assistant, Nicky Telles. Hopefully members are finding that the new website is meeting their expectations and is allowing them to easily access the information that they require. Please email the Webmaster or Web Assistant with suggestions for improvement.

We will soon be moving the site to a host server based in Australia where our banking is done to minimise continuing costs. Apart from a short period of downtime there should be no noticeable change to the site.

Your current webmaster, Joel Hissink, is stepping aside once a replacement Webmaster is found. The Webmaster oversees the development and operation of the website, acts as a conduit between the Executive Committee and the Web Assistant and also sits on the ExCom participating in all ExCom decisions. Please forward expressions of interest with a CV and web experience to the SPUMS President.

\section{Chris Acott awarded Order of Australia}

Dr Chris Acott, FANZCA, DipDHM, Royal Adelaide Hospital, was listed in the Australian Queen's Birthday honours 2017 as a Member (AM) in the general division of the Order of Australia for "significant service to medicine as an anaesthetist, to difficult airway management, to diver safety and to the community". Chris will be known to many in the diving medicine community for his publications in the SPUMS Journal on the Diver Incident Monitoring Study (DIMS). However, other contributions, especially volunteer work as an anaesthetist in Papua New Guinea and Fiji with AusAid, an oral faciomaxillary surgical team, will be less well known. Currently he teaches in PNG on a private basis. Chris was President of SPUMS from 1986-87, 2005-2008 and sits on the ANZCA Overseas Aid Committee. The SPUMS and EUBS Executive Committees congratulate Dr Acott on this meritorious award.

\section{SPTHE}

website is at

<www.spums.org.au>

Members are encouraged to log in. 


\section{SPUMS Diploma in Diving and Hyperbaric Medicine}

\section{Requirements for candidates (May 2014)}

In order for the Diploma of Diving and Hyperbaric Medicine to be awarded by the Society, the candidate must comply with the following conditions:

1 (S)he must be medically qualified, and remain a current financial member of the Society at least until they have completed all requirements of the Diploma.

2 (S)he must supply evidence of satisfactory completion of an examined two-week full-time course in diving and hyperbaric medicine at an approved facility. The list of such approved facilities may be found on the SPUMS website.

3 (S)he must have completed the equivalent (as determined by the Education Officer) of at least six months' full-time clinical training in an approved Hyperbaric Medicine Unit.

4 (S)he must submit a written proposal for research in a relevant area of underwater or hyperbaric medicine, in a standard format, for approval before commencing the research project.

5 (S)he must produce, to the satisfaction of the Academic Board, a written report on the approved research project, in the form of a scientific paper suitable for publication. Accompanying this report should be a request to be considered for the SPUMS Diploma and supporting documentation for 1-4 above.

In the absence of other documentation, it will be assumed that the paper is to be submitted for publication in Diving and Hyperbaric Medicine. As such, the structure of the paper needs to broadly comply with the 'Instructions to authors' available on the SPUMS website $<$ www.spums.org.au $>$ or at $<$ www.dhmjournal.com $>$.

The paper may be submitted to journals other than Diving and Hyperbaric Medicine; however, even if published in another journal, the completed paper must be submitted to the Education Officer (EO) for assessment as a diploma paper. If the paper has been accepted for publication or published in another journal, then evidence of this should be provided.

The diploma paper will be assessed, and changes may be requested, before it is regarded to be of the standard required for award of the Diploma. Once completed to the reviewers' satisfaction, papers not already submitted to, or accepted by, other journals should be forwarded to the Editor of Diving and Hyperbaric Medicine for consideration. At this point the Diploma will be awarded, provided all other requirements are satisfied. Diploma projects submitted to Diving and Hyperbaric Medicine for consideration of publication will be subject to the Journal's own peer review process.

\section{Additional information - prospective approval of projects is required}

The candidate must contact the EO in writing (or email) to advise of their intended candidacy and to discuss the proposed topic of their research. A written research proposal must be submitted before commencement of the research project.

All research reports must clearly test a hypothesis. Original basic and clinical research are acceptable. Case series reports may be acceptable if thoroughly documented, subject to quantitative analysis and if the subject is extensively researched in detail. Reports of a single case are insufficient. Review articles may be acceptable if the world literature is thoroughly analysed and discussed and the subject has not recently been similarly reviewed. Previously published material will not be considered. It is expected that the research project and the written report will be primarily the work of the candidate, and that the candidate is the first author where there are more than one.

It is expected that all research will be conducted in accordance with the joint NHMRC/AVCC statement and guidelines on research practice, available at: <www.nhmrc.gov.au/_files_nhmrc/ publications/attachments/r39.pdf $>$, or the equivalent requirement of the country in which the research is conducted. All research involving humans, including case series, or animals must be accompanied by documentary evidence of approval by an appropriate research ethics committee. Human studies must comply with the Declaration of Helsinki (1975, revised 2013). Clinical trials commenced after 2011 must have been registered at a recognised trial registry site such as the Australia and New Zealand Clinical Trials Registry <http://www.anzctr.org.au/> and details of the registration provided in the accompanying letter. Studies using animals must comply with National Health and Medical Research Council Guidelines or their equivalent in the country in which the work was conducted.

The SPUMS Diploma will not be awarded until all requirements are completed. The individual components do not necessarily need to be completed in the order outlined above. However, it is mandatory that the research proposal is approved prior to commencing research.

Projects will be deemed to have lapsed if:

- the project is inactive for a period of three years, or

- the candidate fails to renew SPUMS Membership in any year after their Diploma project is registered (but not completed).

For unforeseen delays where the project will exceed three years, candidates must explain to the EO by email why they wish their diploma project to remain active, and a three-year extension may be approved. If there are extenuating circumstances why a candidate is unable to maintain financial membership, then these must be advised by email to the EO for consideration by the SPUMS Executive. If a project has lapsed, and the candidate wishes to continue with their DipDHM, then they must submit a new application as per these guidelines.

The Academic Board reserves the right to modify any of these requirements from time to time. As of January 2016, the SPUMS Academic Board consists of:

Dr David Wilkinson, Education Officer, Adelaide;

Professor Simon Mitchell, Auckand;

Dr Denise Blake, Townsville.

All enquiries and applications should be addressed to: David Wilkinson

education@spums.org.au

Key words

Qualifications; Underwater medicine; Hyperbaric oxygen; Research; Medical society 


\section{Royal Adelaide Hospital Medical Officers' Course in Diving and Hyperbaric Medicine 2018}

Dates: 28 August-08 September

Venue: The Royal Adelaide Hospital, Adelaide

Cost: AUD2,500.00 (inclusive of GST)

Course Conveners: David Wilkinson and Suzy Szekely Invited faculty includes: Professors Michael Bennett and Simon Mitchell

The course content includes:

- Physics and physiology of diving

- Recreational fitness-to-dive

- Occupational fitness-to-dive

- Decompression illness and non-dysbaric injuries

- Medical management and return to diving

- Technical and professional diving

- Marine envenomation

- Introduction to hyperbaric medicine

\section{Contact for information:}

Ms Lorna Mirabelli, Course Administrator

Phone: +61-(0)8-8222-5116

E-mail: <Lorna.Mirabelli@sa.gov.au>

\section{ANZ Hyperbaric Medicine Group Introductory Course in Diving and Hyperbaric Medicine 2018}

Dates: 12-23 February

Venue: Fiona Stanley Hospital and Esplanade Hotel, Fremantle, Western Australia

Cost: AUD2,500 (inclusive of GST)

Course Conveners: Ian Gawthrope and Neil Banham

The Course content includes:

- History of diving medicine and hyperbaric oxygen

- Physics and physiology of diving and compressed gases

- Presentation, diagnosis and management of diving injuries

- Assessment of fitness to dive

- Visit to RFDS base for flying and diving workshop

- Accepted indications for hyperbaric oxygen treatment

- Hyperbaric oxygen evidence-based medicine

- Wound management and transcutaneous oximetry

- In water rescue and management of a seriously ill diver

- Visit to HMAS Stirling

- Practical workshops

- Marine Envenomation

\section{Contact for information:}

Sue Conlon, Course Administrator

Phone: +61-(0)8-6152-5222

E-mail: <fsh.hyperbaric@ health.wa.gov.au>

\section{Scott Haldane Foundation}

As an institute dedicated to education in diving medicine, the Scott Haldane Foundation has organized more than 240 courses over the past 20 years. SHF is targetting more and more on an international audience with courses worldwide.

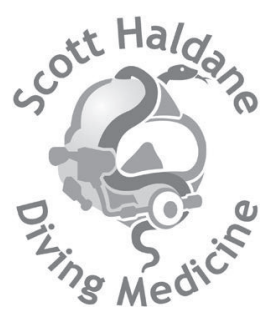

The courses Medical Examiner of Diver (part I and II) and SHF in-depth courses, as modules of the level 2d Diving Medicine Physician course, fully comply with the ECHM/ EDTC curriculum for Level 1 and $2 d$ respectively and are accredited by the European College of Baromedicine (ECB).

\section{SHF Course Calendar 2017}

04-11 November: Basic course part 1, Flores/Komodo, Indonesia

11-18 November: $N E W$ SHF In-depth course "Lungs in the abyss", Flores/Komodo, Indonesia

18-25 November: NEW SHF In-depth course "Lungs in the abyss", Flores/Komodo, Indonesia

\section{First half 2018}

23, 24 March: Basic course part 1, Zeist, NL

7, 13 \& 14 April: Basic course part 2, Amsterdam, NL

1st half 2018: Basic course part 2, Bonaire; tbc

1st half 2018: HBOT and decompression; tbc

On request: Internship different types of diving (DMP certification), NL

On request: Internship hyperbaric medicine (DMP certification), NL/Belgium

For further information: <www.scotthaldane.org $>$

British Hyperbaric Association Annual Scientific Meeting 2017

Dates: 20-21 October

Venue: Jury's Inn, Birmingham

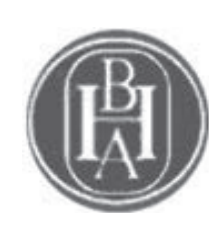

The meeting will be held jointly with the UK Diving Medical Committee and will be aligned to refresher training for HSE Approved Medical Examiners of Divers. The Dive Show is also being held in Birmingham 21-22 October.

For more information: < http://www.ukhyperbaric.com/ meetings/2017-annual-scientific-meeting-and-agm/> 


\section{Capita Selecta Diving Medicine Academic Medical Centre, University of Amsterdam, The Netherlands}

Saturday 04 November: Symposium on diving medicine: Disorders of the head, from ophthalmology to psychology

Topics will include: Human error and diver performance, psychiatric, central neurological, visual, hearing and vestibular disorders, Speakers include Gareth Lock, UK, Cees Meeuwis, NL and Bram Querido, NL.The level of this advanced course is accordance with that of EDTC/ECHM for Medical Examiner (Level 1) and Diving Physician (Level 2D). Accreditation is expected to be granted by your national accreditation institutions. A prior basic course in diving medicine is a prerequisite for registration.

Register by using this link:

$<$ www.capitaselectaduikgeneeskunde.nl>

\section{Hyperbaric Oxygen, Karolinska}

Welcome to: <http://www.hyperbaricoxygen.se/> This site, supported by the Karolinska University Hospital, Stockholm, Sweden, offers publications and high-quality lectures from leading investigators in hyperbaric medicine. Please register to obtain a password via e-mail. Once registered, watch online, or download to your iPhone, iPad or computer for later viewing.

For further information contact:

E-mail: <folke.lind@karolinska.se>

\section{German Society for Diving and Hyperbaric Medicine (GTÜeM)}

An overview of basic and refresher courses in diving and hyperbaric medicine, accredited by GTÜeM according to EDTC/ECHM curricula, can be found on the website: <http://www.gtuem.org/212/Kurse_/_Termine/Kurse.html>

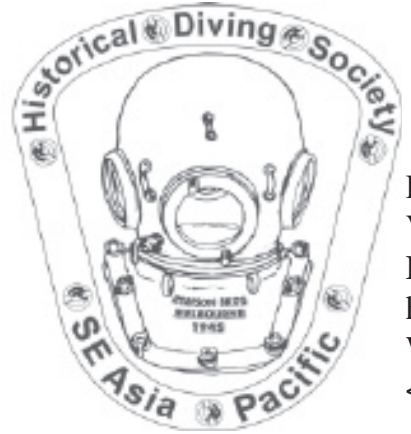

\section{DIVING HISTORICAL SOCIETY AUSTRALIA, SE ASIA}

P O Box 347, Dingley Village Victoria, 3172, Australia E-mail:<hdsaustraliapacific@ hotmail.com.au>

Website:

$<$ www.classicdiver.org $>$

\section{The Science of Diving}

Support EUBS by buying the PHYPODE book "The science of diving". Written for anyone with an interest in the latest research in diving physiology and pathology. The royalties from this book are being donated to the EUBS.

Need more reason to buy? We don't think so!

Available from: Morebooks <https://www.morebooks. $\mathrm{de} / \mathrm{store} / \mathrm{gb} / \mathrm{book} /$ the-science-of-diving/isbn/978-3-65966233-1>

\section{Advertising in Diving and Hyperbaric Medicine}

Companies and organisations within the diving, hyperbaric medicine and wound-care communities wishing to advertise their goods and services in Diving and Hyperbaric Medicine are welcome. The advertising policy of the parent societies appears on the journal website:

$<$ www.dhmjournal.com>

Details of advertising rates and formatting requirements are available on request from:

E-mail: <editorialassist@dhmjournal.com>

\section{Copyright}

All articles in Diving and Hyperbaric Medicine are published under licence from the authors. Copyright to these articles remains with these authors. Any distribution, apart from for limited educational purposes, is in breach of copyright.

\section{Instructions for authors}

A downloadable pdf of the 'Instructions to authors' (revised July 2017) and other guidance for preparing a submission can be found on the Diving and Hyperbaric Medicine (DHM) website: $<$ www.dhmjournal.com $>$. Authors must read and follow these instructions carefully.

All submissions to $D H M$ should be made using the portal at <http://www.manuscriptmanager.com/dhm>. Before submitting, please view the video on how to prepare a submission at: <https://www.youtube.com/ watch? $=$ gpMsPAX $4 p W A \& t=41 \mathrm{~s}>$.

In case of difficulty, please contact the Editorial Assistant by e-mail at: <editorialassist@dhmjournal.com>.

\section{DAN Europe}

DAN Europe has a fresh, multilingual selection of recent news, articles and events featuring DAN and its staff.

Go to the website: $<$ http://www.daneurope.org/web/guest/> 




\section{DIVER EMERGENCY SERVICES PHONE NUMBERS}

\author{
AUSTRALIA \\ 1800-088200 (in Australia, toll-free) \\ +61-8-8212-9242 (International) \\ NEW ZEALAND \\ 0800-4DES-111 (in New Zealand, toll-free) \\ +64-9-445-8454 (International) \\ ASIA \\ +81-3-3812-4999 (Japan)
}

\author{
SOUTHERN AFRICA \\ 0800-020111 (in South Africa, toll-free) \\ +27-828-106010 (International, call collect) \\ EUROPE \\ +39-6-4211-8685 (24-hour hotline) \\ UNITED KINGDOM \\ +44-7740-251-635
}

USA

+1-919-684-9111

The DES numbers (except UK) are generously supported by DAN

\section{DAN ASIA-PACIFIC DIVE ACCIDENT REPORTING PROJECT}

This project is an ongoing investigation seeking to document all types and severities of diving-related incidents. All information is treated confidentially with regard to identifying details when utilised in reports on fatal and non-fatal cases. Such reports may be used by interested parties to increase diving safety through better awareness of critical factors.

Information may be sent (in confidence unless otherwise agreed) to:

DAN Research

Divers Alert Network Asia Pacific

PO Box 384, Ashburton VIC 3147, Australia

Enquiries to e-mail: <research@danasiapacific.org>

\section{DAN Asia-Pacific NON-FATAL DIVING INCIDENTS REPORTING (NFDIR)}

NFDIR is an ongoing study of diving incidents, formerly known as the Diving Incident Monitoring Study (DIMS). An incident is any error or occurrence which could, or did, reduce the safety margin for a diver on a particular dive. Please report anonymously any incident occurring in your dive party. Most incidents cause no harm but reporting them will give valuable information about which incidents are common and which tend to lead to diver injury. Using this information to alter diver behaviour will make diving safer.

The NFDIR reporting form can be accessed on line at the DAN AP website: $<$ www.danasiapacific.org/main/accident/nfdir.php>

\section{DISCLAIMER}

All opinions expressed in this publication are given in good faith and in all cases represent the views of the authors and are not necessarily representative of the policies or views of the SPUMS, EUBS or the Editor and Board. 


\section{CONTENTS}

Diving and Hyperbaric Medicine Volume 47 No. 3 September 2017

\section{Editorials}

141 The Editor's offering

142 The Presidents' pages

\section{Original articles}

144 Chain of events analysis for a scuba diving fatality John Lippmann, Christopher Stevenson, David McD Taylor, Jo Williams, Mohammadreza Mohebbi

155 Heat deactivation of the stonefish Synanceia horrida venom - implications for first-aid management

Samantha Barnett, Silvia Saggiomo, Michael Smout, Jamie Seymour

159 Decompressing rescue personnel during Australian submarine rescue operations

Michael P Reid, Andrew Fock, David J Doolette

168 Decompressing recompression chamber attendants during Australian submarine rescue operations Michael P Reid, Andrew Fock, David J Doolette

173 Turkish recreational divers: a comparative study of their demographics, diving habits, health and attitudes towards safety

Bengusu Mirasoglu, Samil Aktas

180 Diving with pre-existing medical conditions

John Lippmann, David McD Taylor, Christopher Stevenson, Jo Williams, Simon J Mitchell

\section{Technical report}

191 A pleural vacuum relief device for pleural drain unit use in the hyperbaric environment

Marco Gelsomino, Theo Tsouras, Ian Millar, Andrew Fock

\section{Case reports}

198 Periorbital emphysema after a wet chamber dive Wei-Shih Tseng, Hui-Chieh Lee, Bor-Hwang Kang

201 Ruptured tympanic membrane from underwater impact with an Atlantic salmon, Salmo salar David Smart

\section{Letters to the Editor}

203 Proliferative retinopathy during hyperbaric oxygen treatment Viet Tran, David Smart

203 Advertising policy for Diving and Hyperbaric Medicine Jacek Kot, David Smart

205 Tricon2018 - Durban, South Africa

\section{EUBS notices and news}

206 EUBS Member at Large

206 ECHM Level 3 (Expert in Hyperbaric Medicine) recognition

206 European Wound Management Association (EWMA) position paper on oxygen therapies

206 Open Letter to the American Diabetes Association Board of Directors

\section{SPUMS notices and news}

207 Australian and New Zealand College of Anaesthetists news

207 Chris Acott awarded Order of Australia

208 SPUMS Diploma in Diving and Hyperbaric Medicine

209 Courses and meetings

Diving and Hyperbaric Medicine is indexed on MEDLINE, SciSearch ${ }^{\circledR}$ and Embase/Scopus 